\title{
YEARLY VARIATIONS IN RUNOFF AND FREQUENCY OF DRY YEARS FOR THE CONTERMINOUS \\ UNITED STATES, 1911—79
}

By W.B. Langbein and J.R. Slack

\section{U.S. GEOLOGICAL SURVEY}

Open-File Report 82-751

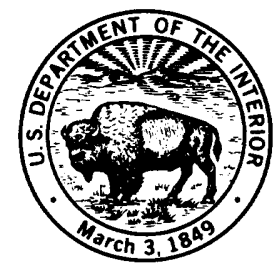




\title{
UNITED STATES DEPARTMENT OF THE INTERIOR JAMES G. WATT, Secretary
}

\author{
GEOLOGICAL SURVEY \\ Dallas L. Peck, Director
}

For further information write to:

Chief Hydrologist

U.S. Geological Survey

410 National Center

Reston, Virginia 22092 
Abstract. . . . . . . . . . . . . . . . . . .

Introduction. . . . . . . . . . . . . . . . . .

FIGURES

Figure

Page

1-69 Annual runoff in percent of normal for the water years 1911 through 1979 ..............

70 Grid plan superposed on conterminous United States. . .

71 Frequency of dry years per decade, West, Center, and East regions......................

72 Distribution of years having indicated areal extent of subnormal runoff....................

73 Frequency of dry years per decade in North and South parts of West region . . . . . . . . . . . . . .

\section{TABLES}

Table

Page

1 Gaging station records used in this report. . . . . .

2 Percent of cells in each region with subnormal runoff by water years . . . . . . . . . . . . . . .

3 Number of dry years by decades for each region and for the conterminous United States as a whole . . . . .

References. . . . . . . . . . . . . . . . . 


\author{
Yearly Variations in Runoff and Frequency of Dry Years \\ for the Conterminous United States, 1911-79 \\ By W. B. Langbein and J. R. Slack
}

\title{
ABSTRACT
}

This report presents gaging station data on variations in annual runoff over the conterminous United States and nearby Canada during the period 1911-79 in tabular and graphic (map) form. These data bring up to date and extend backward in time previous reports on streamflow stations by Harbeck and Langbein in 1949 which covered the period 1921-45 and that by Busby in 1963 which covered the period 1931-60. As depicted by Langbein and S1ack in 1980, these data show that, contained within a rather large seemingly random or sporadic pattern, there appears to be a previously unnoticed degree of similarity in the occurrence of subnormal flows across the country. In each of three regions, viz., West, Center, and East, dry years were concentrated in two sequences, one centered in the 1930's and the second in the decade from the mid-50's to the mid-60's.

\section{INTRODUCTION}

The computer-based maps shown in figures 1-69 give the annual runoff as a fraction of normal (defined below) for each water year from 1911 through 1979. Streamflow records at 211 stream-gaging stations (table 1) were used to provide the data on those maps. To compile the records for this purpose, we began by using the stations used by Busby (1963, table 1) for the 1931-60 period. It was found that few of these records extended back to 1911 and that many stations were either discontinued after 1960 or had become disqualified for the purposes of this report because of excessive regulation or diversions. It was necessary to look for other records. This search for usable records to cover the annual maps to the same degree as previously made necessary the inclusion of records of streamflow that are altered to some degree by regulation or diversion giving preference to those least so affected. Station records were classified as follows in this regard.

Class I - No reported regulation or diversion

Class II - Diversion less than ten percent of the mean flow and storage capacity less than ten percent of the mean annual runoff.

\section{Class III - All others}

Gaging-station records of Classes I and II were given priority; those of Class III were excluded except where needed to fill 1 arge areal gaps. Only 27 of such stations were included; all were in the central or western regions of the country. There was no drainage area criterion except that, where there was a multiple choice among stations otherwise acceptable, preference was given to those streams having drainage areas between 100 and 10,000 square 


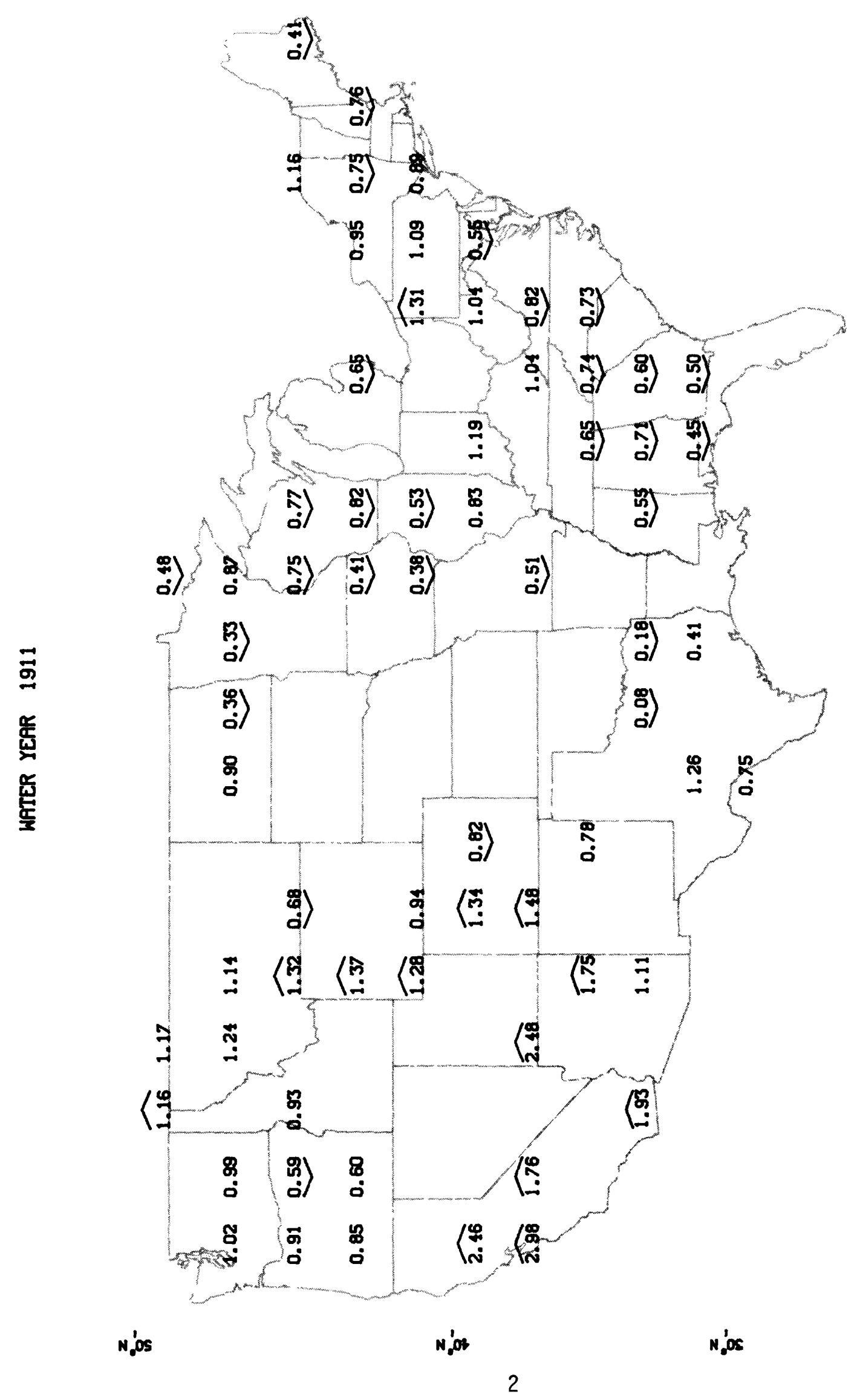

$-9$

$-\frac{7}{2}$

$-\frac{x}{9}$

$-8$

$-2$

r. 


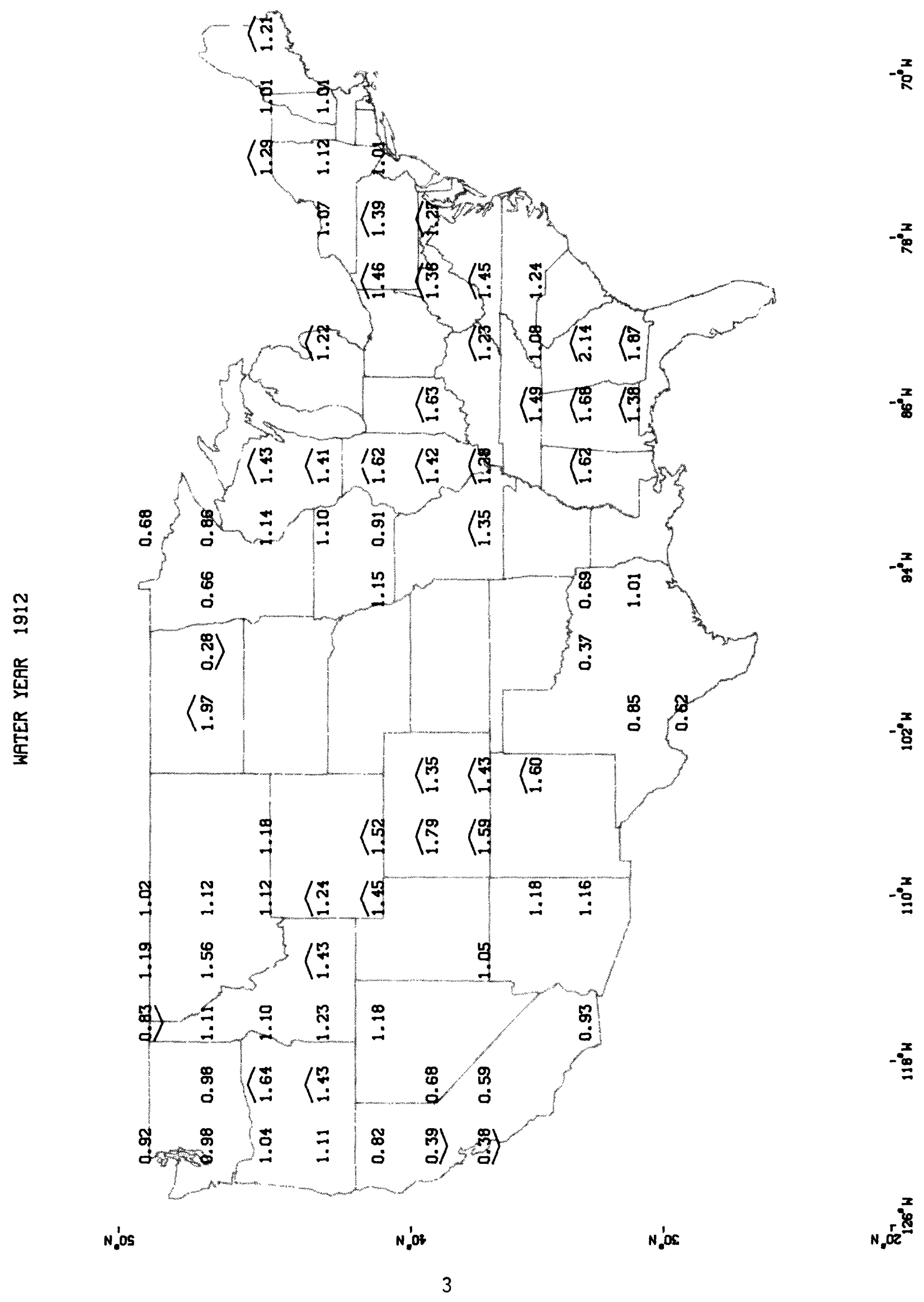




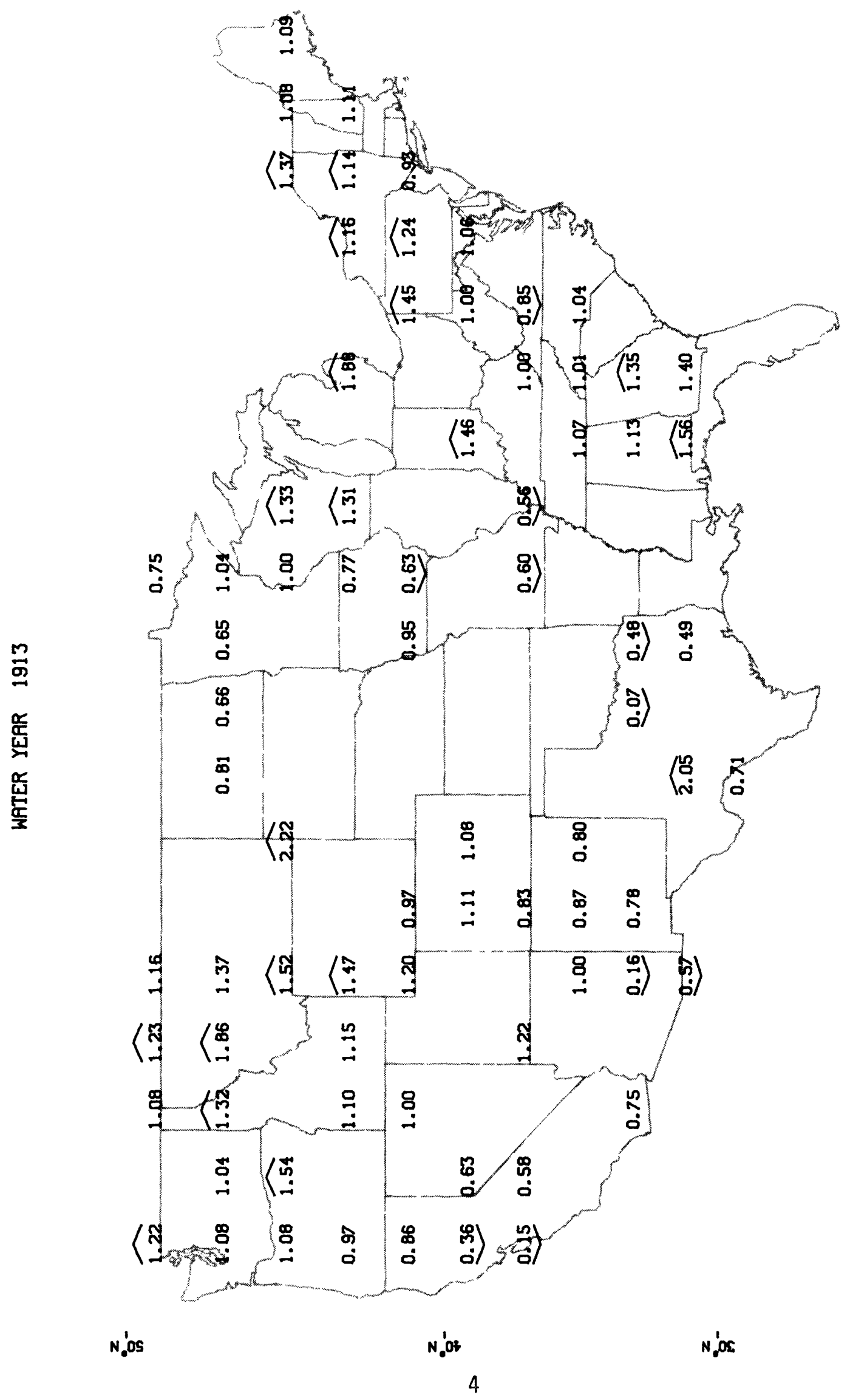

$-\frac{7}{2}$

$-\frac{7}{8}$

$-\frac{7}{8}$

$-\frac{7}{6}$

$\begin{array}{r}7 \\ -8 \\ \hline\end{array}$

$-8$

$-\underset{0}{-9}$

$\underbrace{x}_{02}$ 


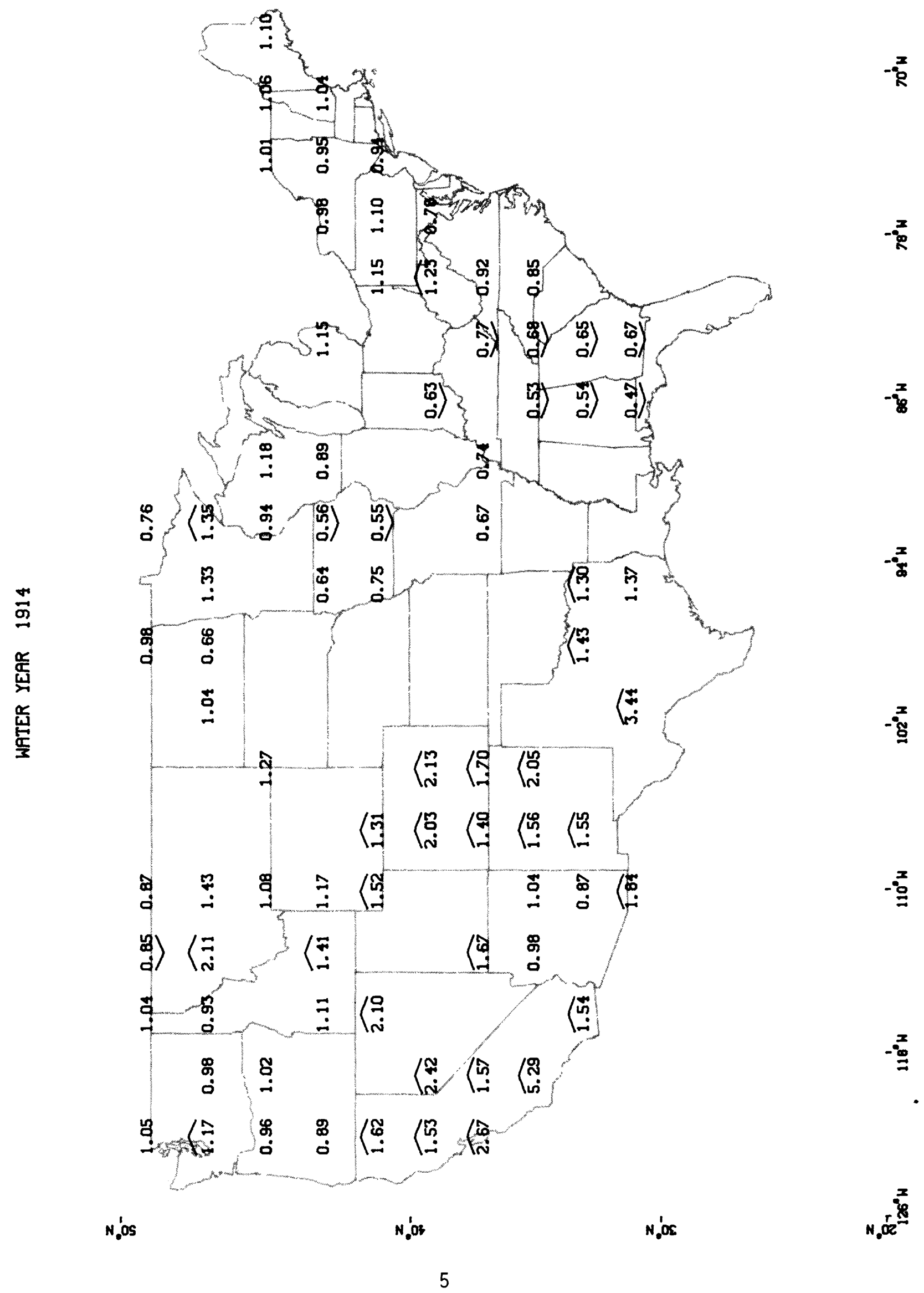




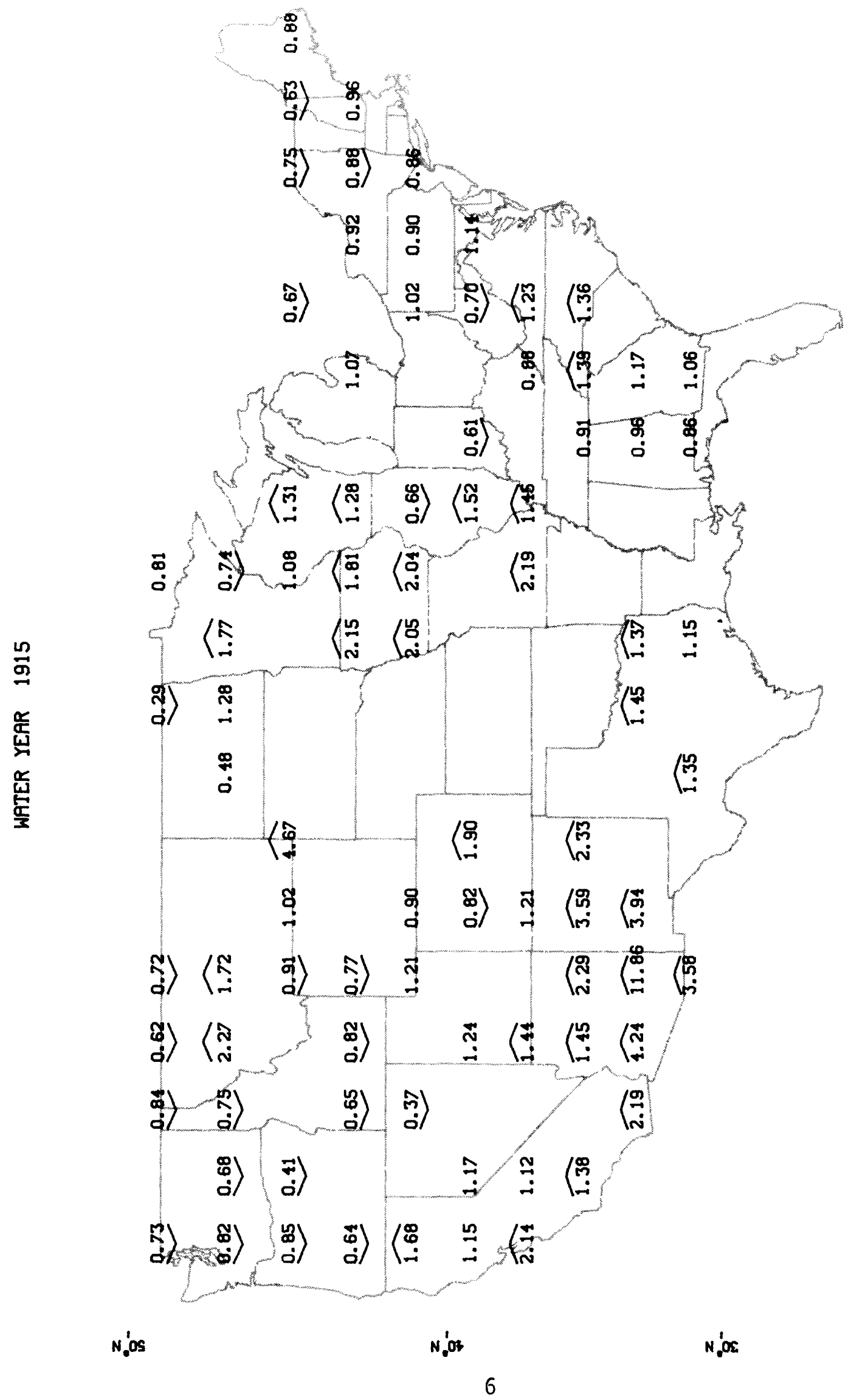




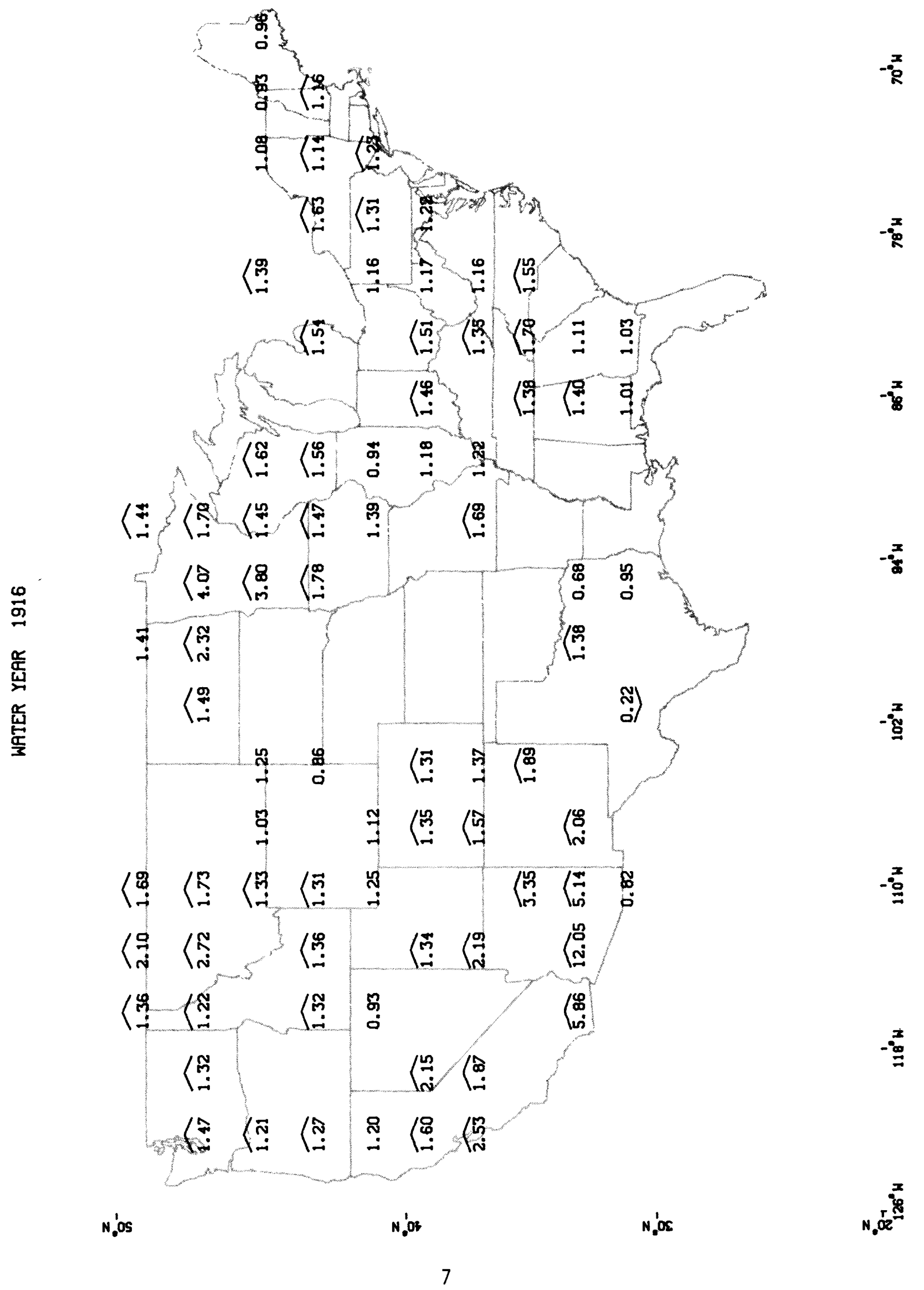




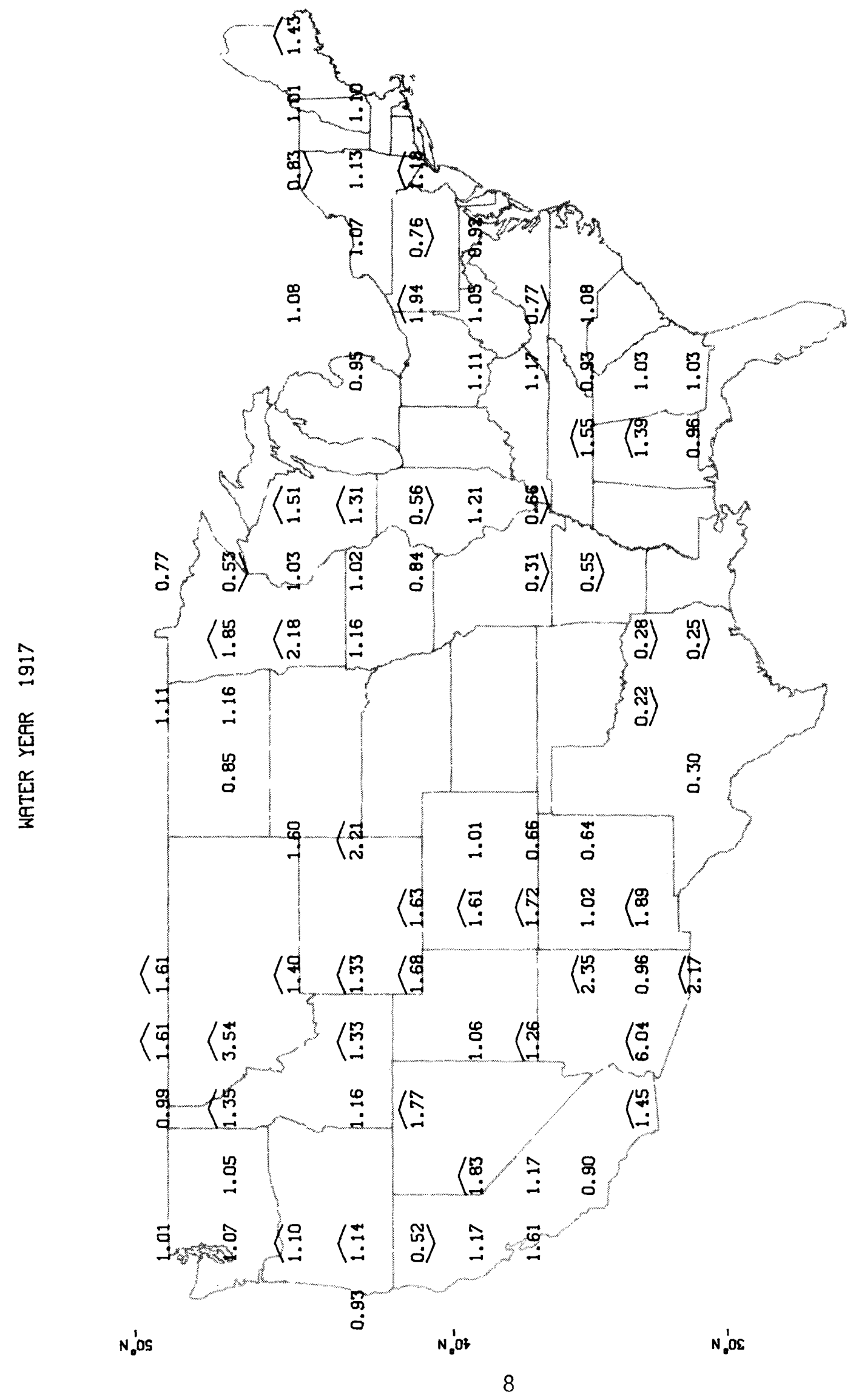




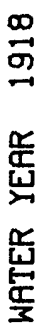

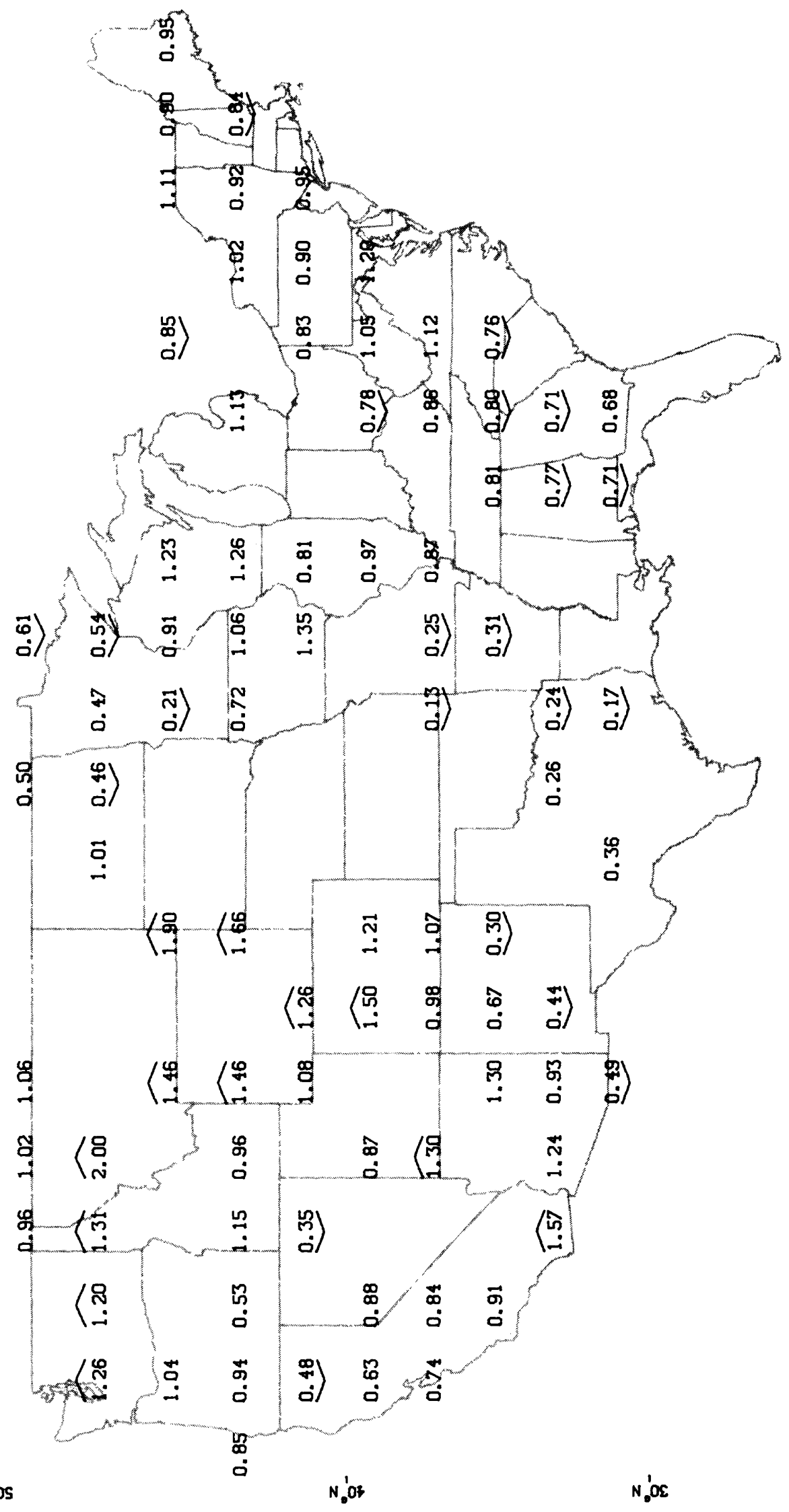

$-\frac{7}{8}$

$-\frac{7}{5}$

- ำ

$-\frac{x}{-0}$

- 


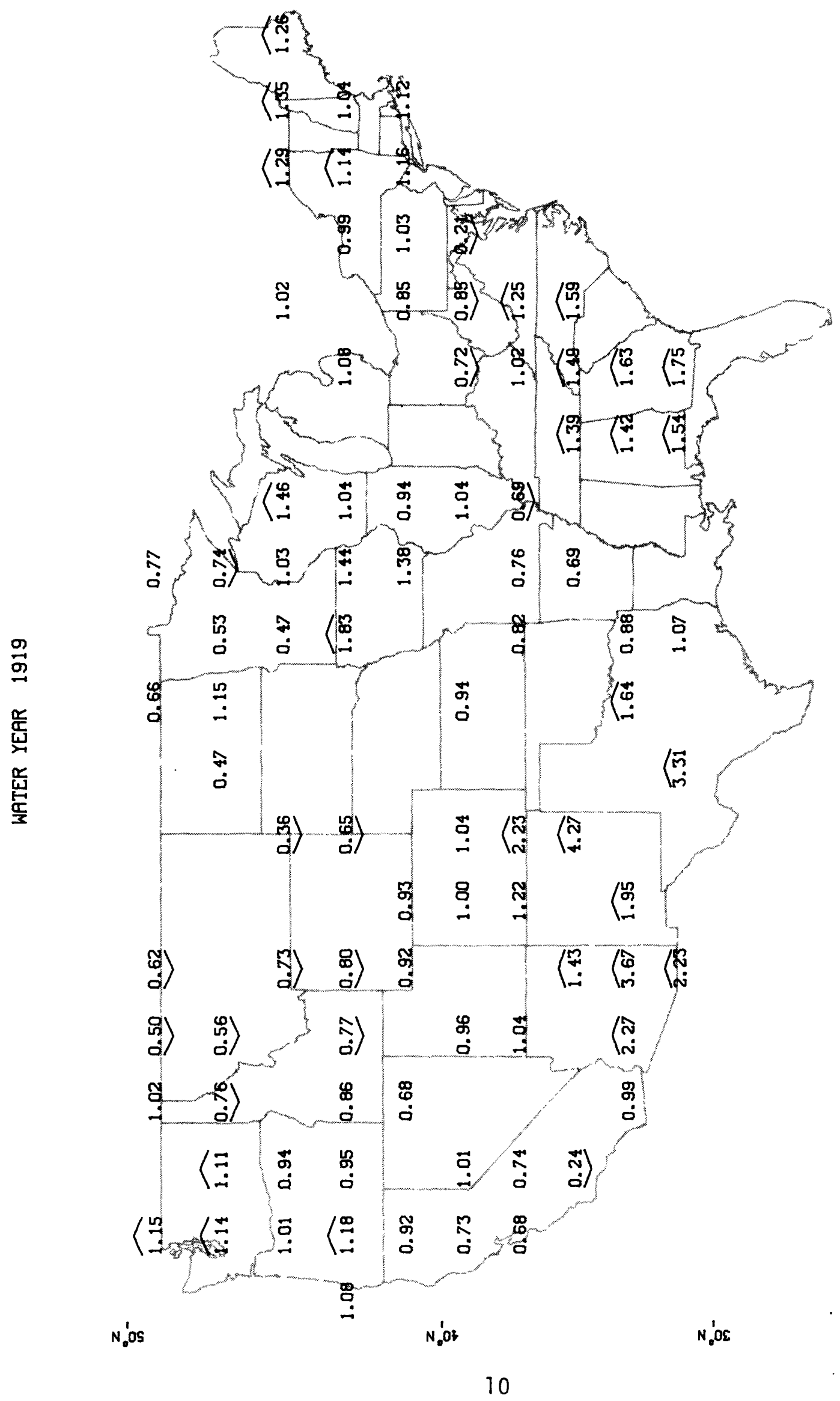




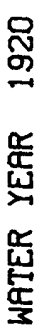

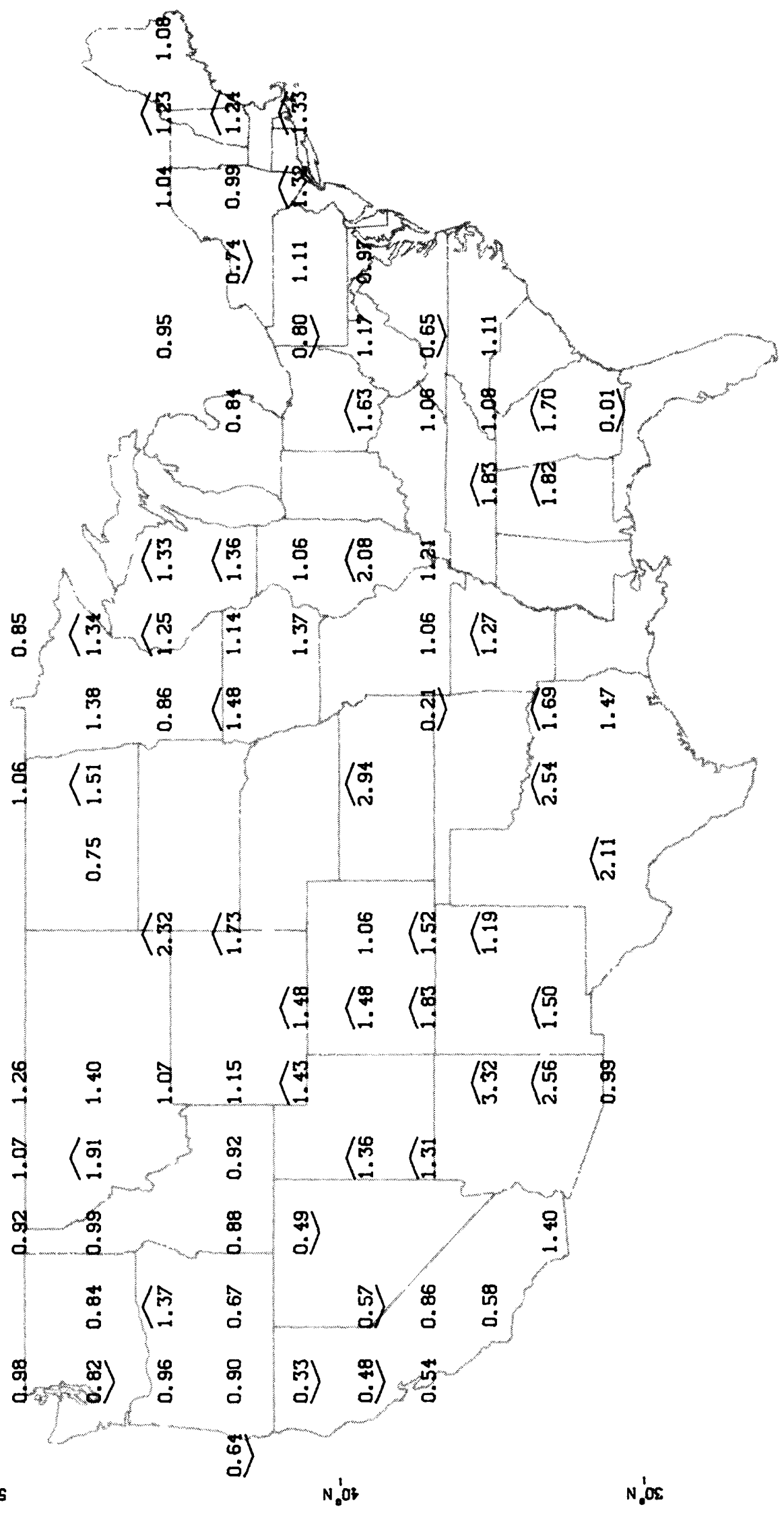

$-\frac{2}{8}$

$-\frac{7}{\infty}$

$-\frac{7}{8}$

$-\frac{7}{5}$

-

$-\stackrel{7}{0}$

$-\stackrel{7}{-\infty}$ 


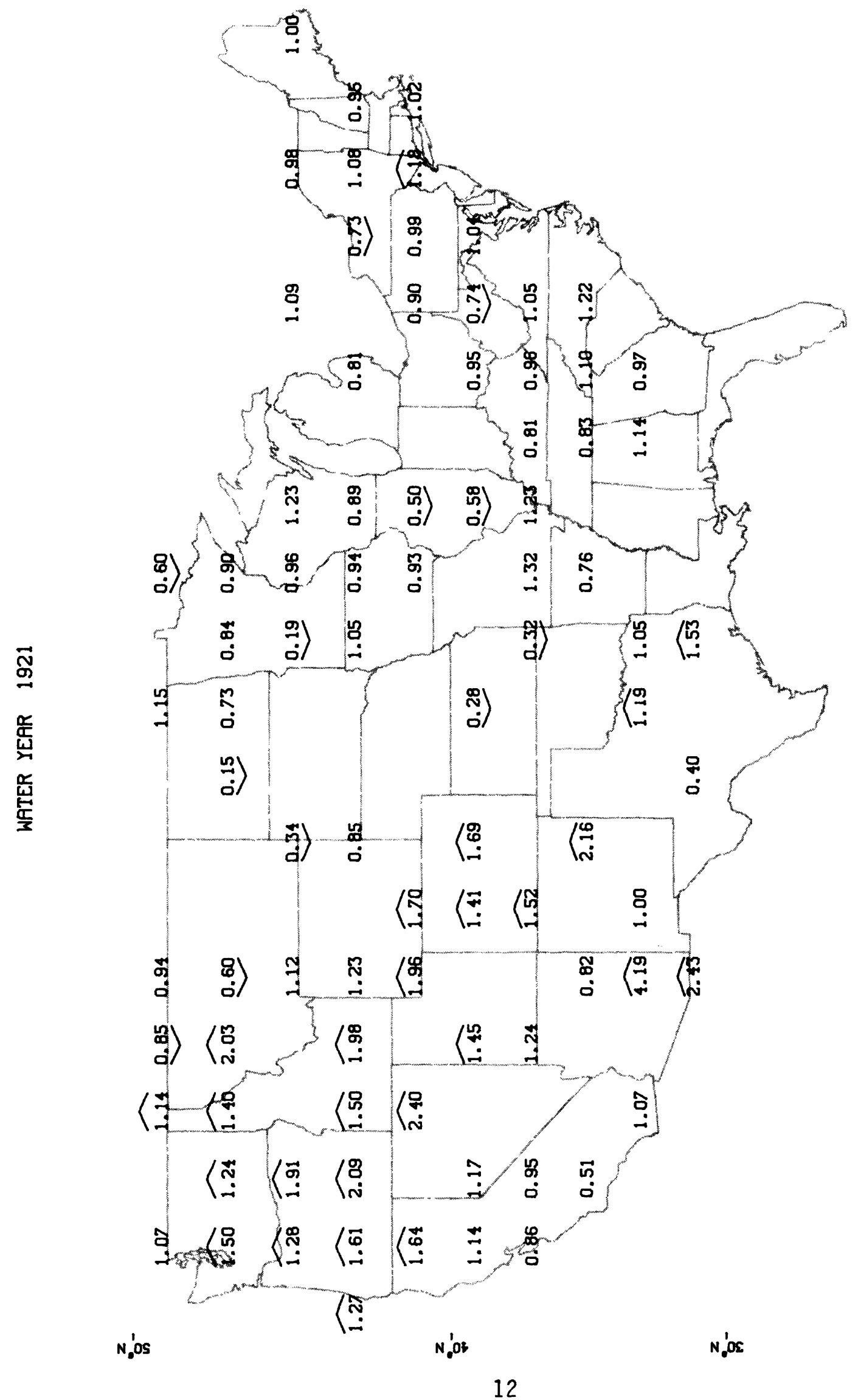




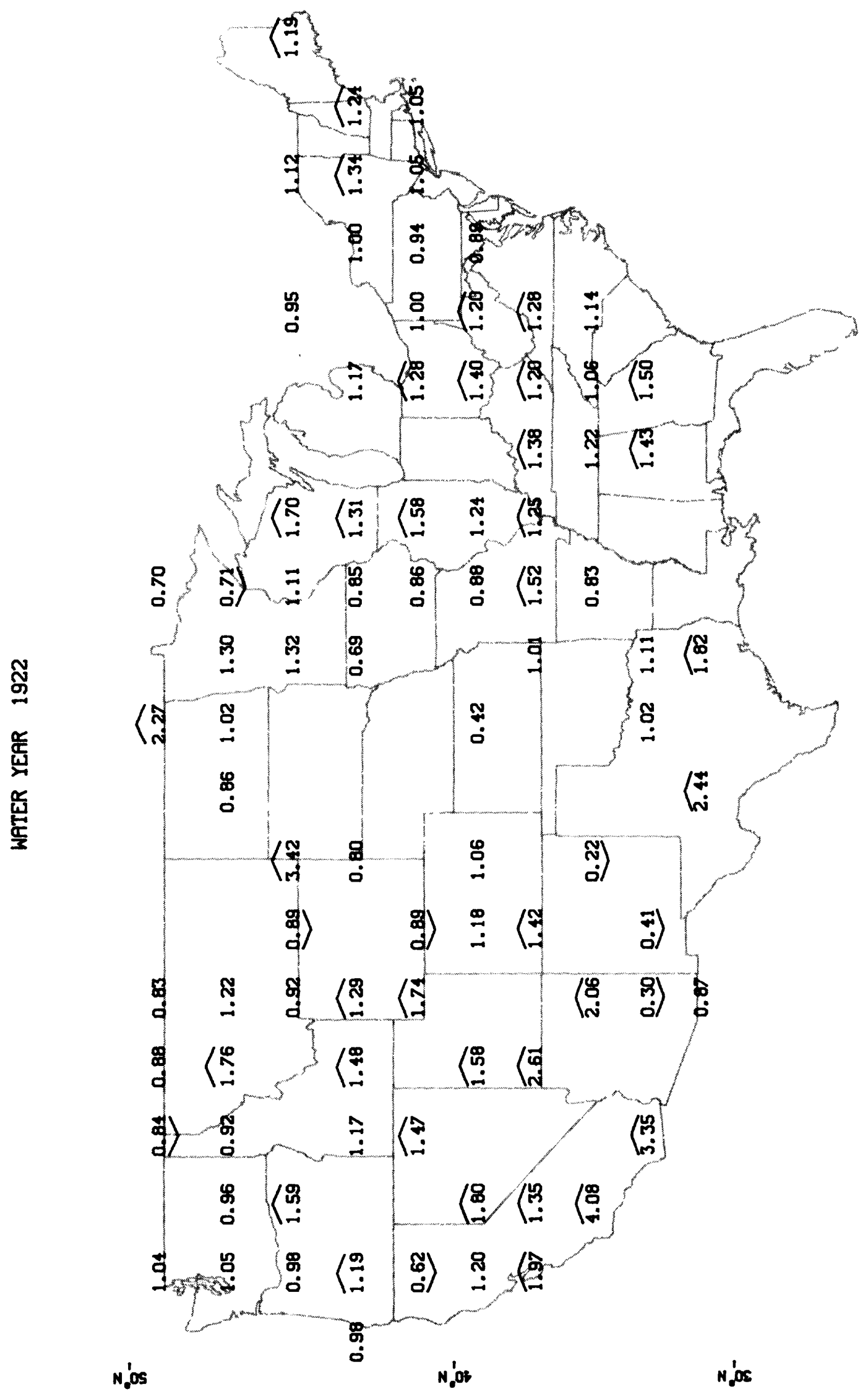

$-\frac{x}{2}$

$-\frac{x}{8}$

$-\frac{x}{8}$

$-\frac{x}{11}$

$-\frac{x}{8}$

-

$\underset{-\infty}{-\infty}$ 


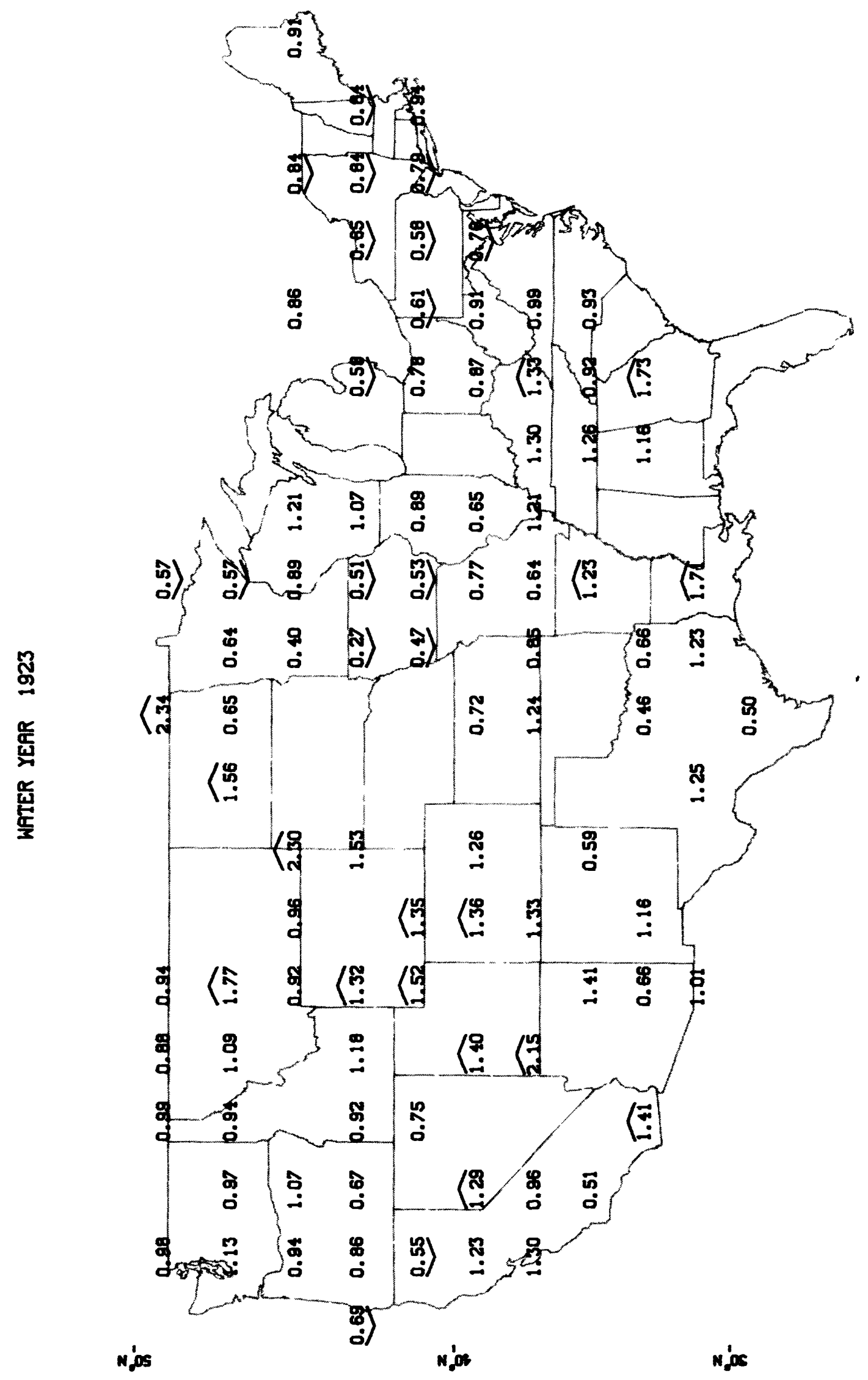




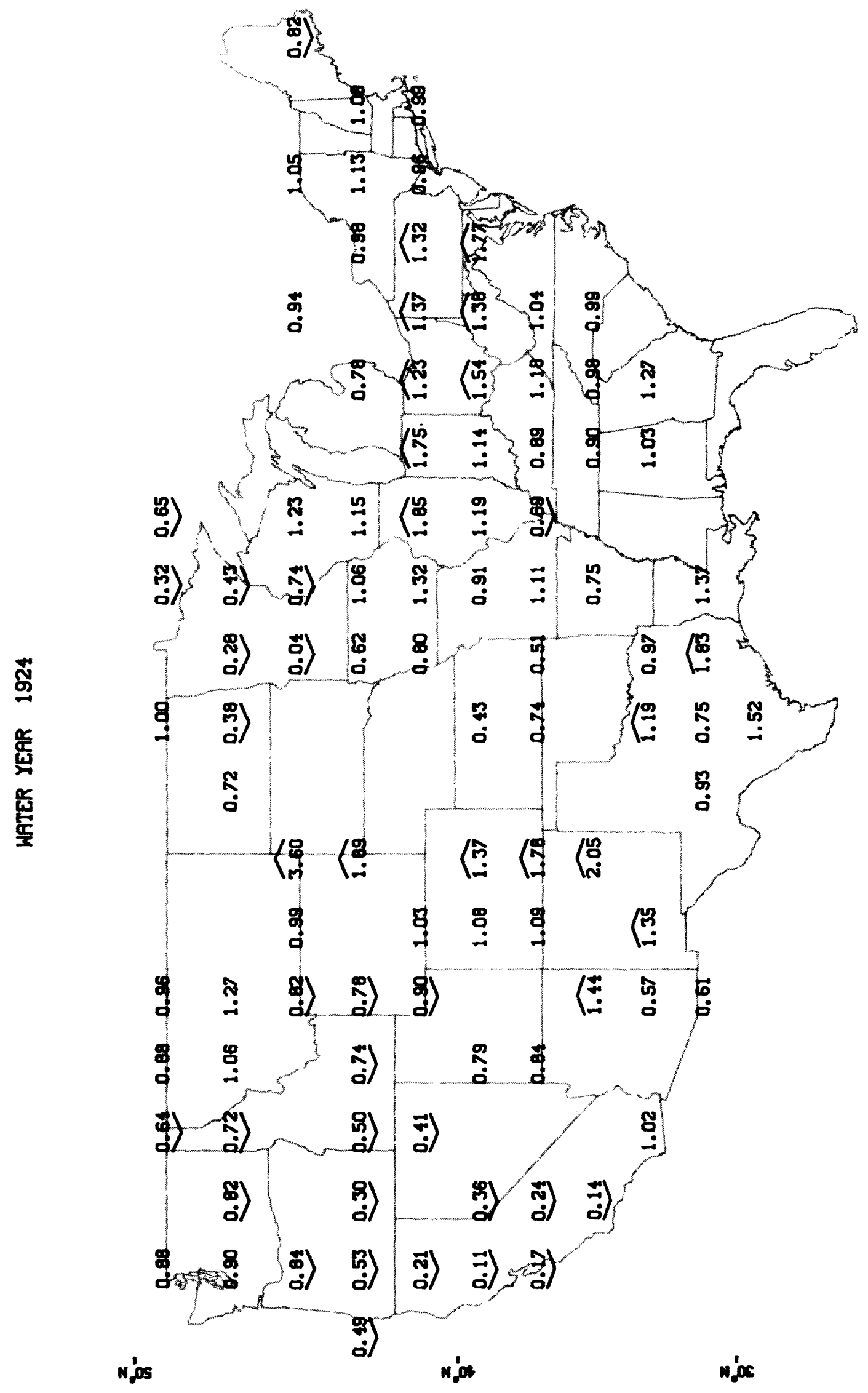




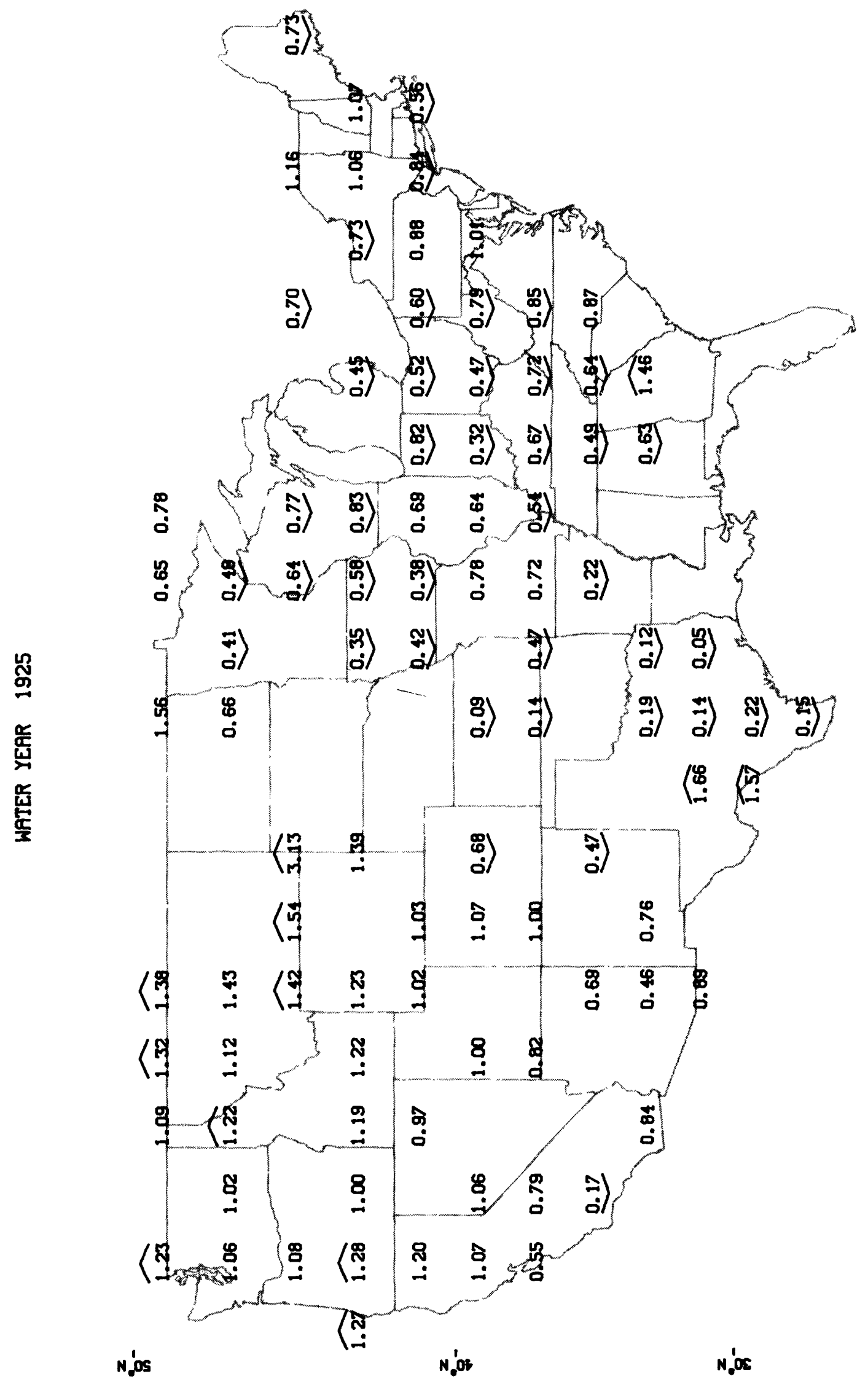

$-\frac{x}{R}$

$-2$

$-2$

$-5$

$-\frac{2}{8}$

$-\stackrel{x}{-2}$

$-\underset{0}{-2}$ 


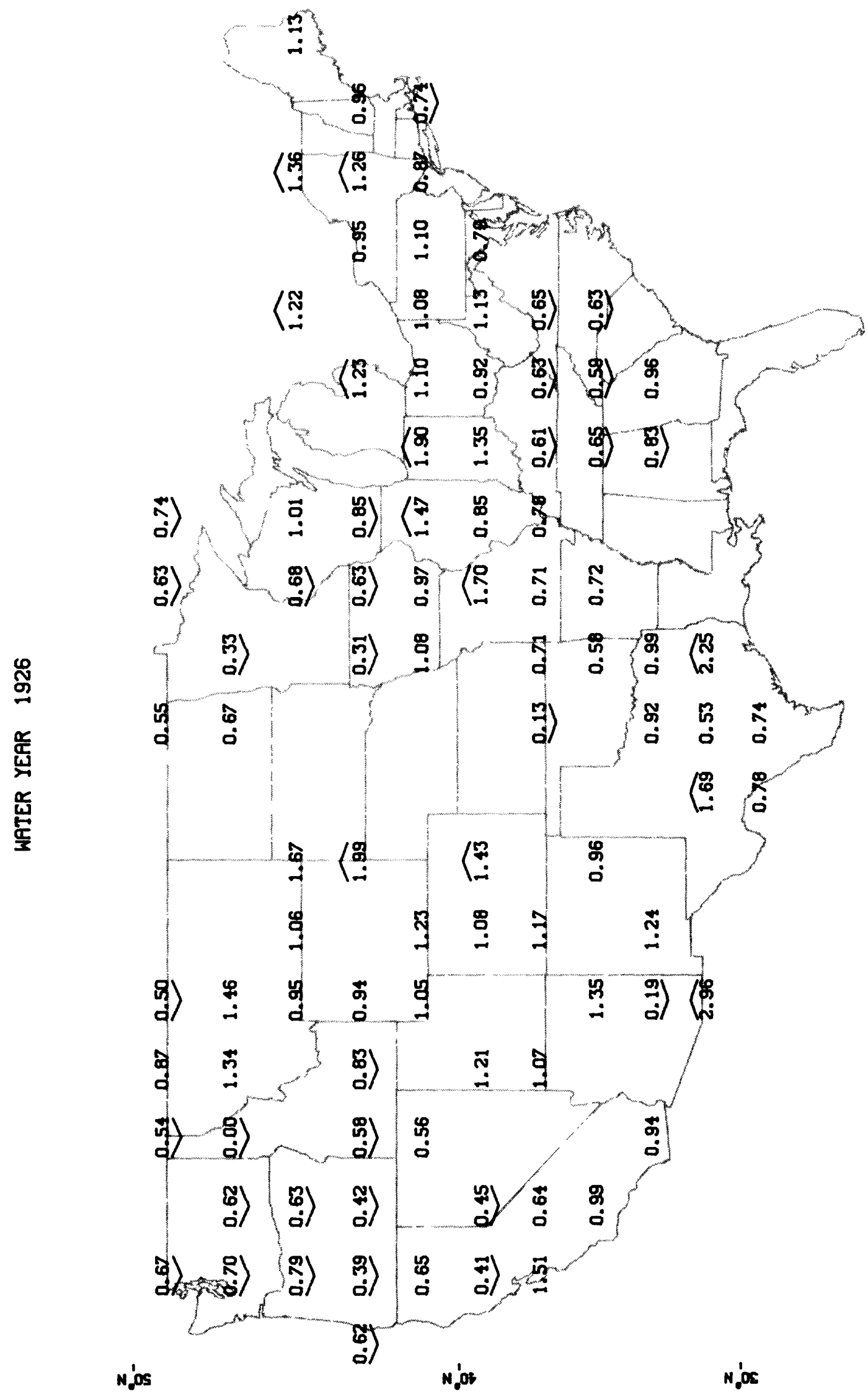

$-\frac{x}{2}$

$-\frac{x}{2}$

$-\frac{x}{8}$

$-\frac{x}{5}$

$-8$

$-\frac{x}{0}$

$-\frac{9}{7}$ 


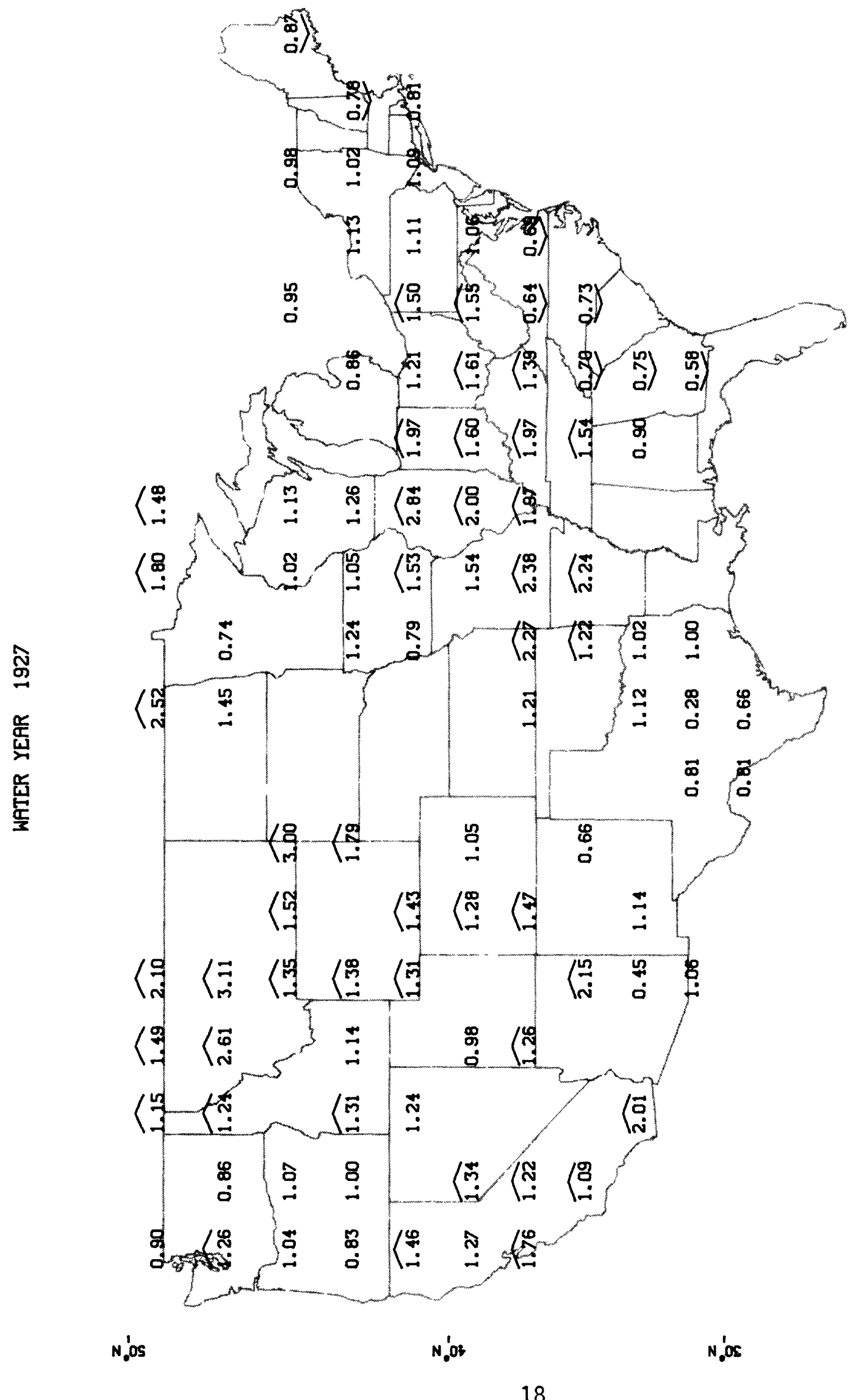




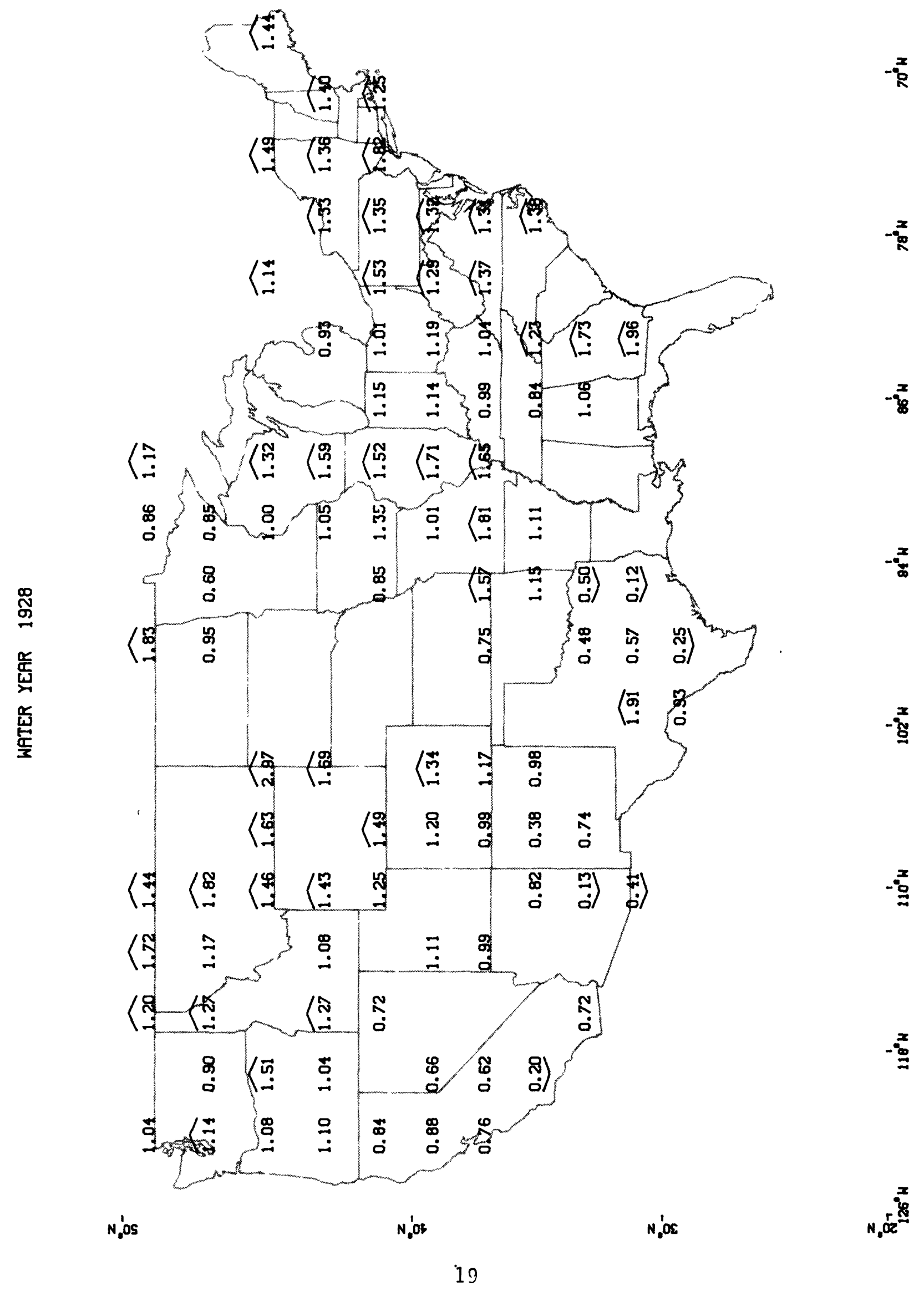




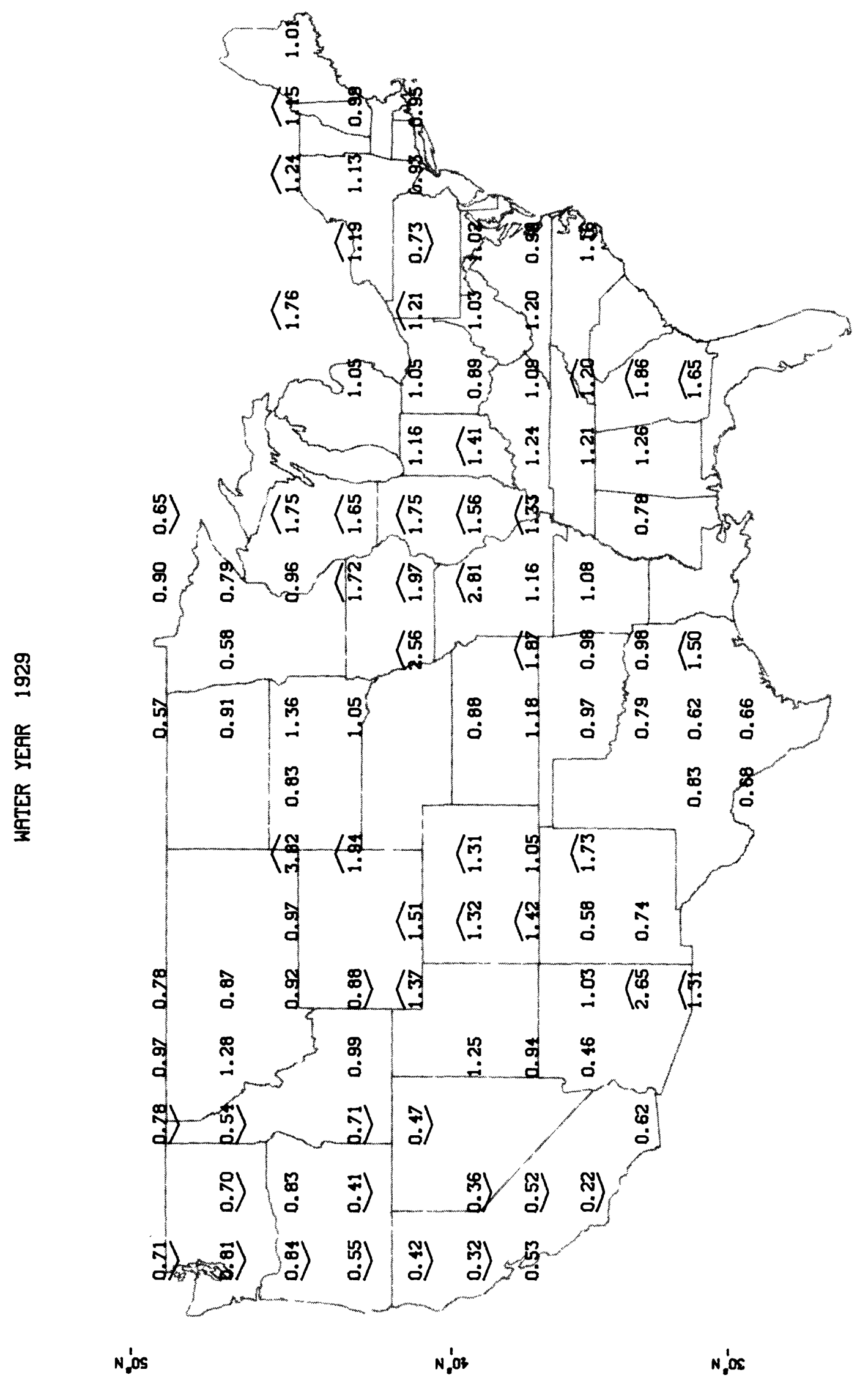

$-\frac{x}{2}$

$-\frac{x}{8}$

$-\frac{7}{8}$

$-\frac{7}{15}$

$-\frac{x}{9}$

$-\frac{x}{2}$

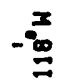




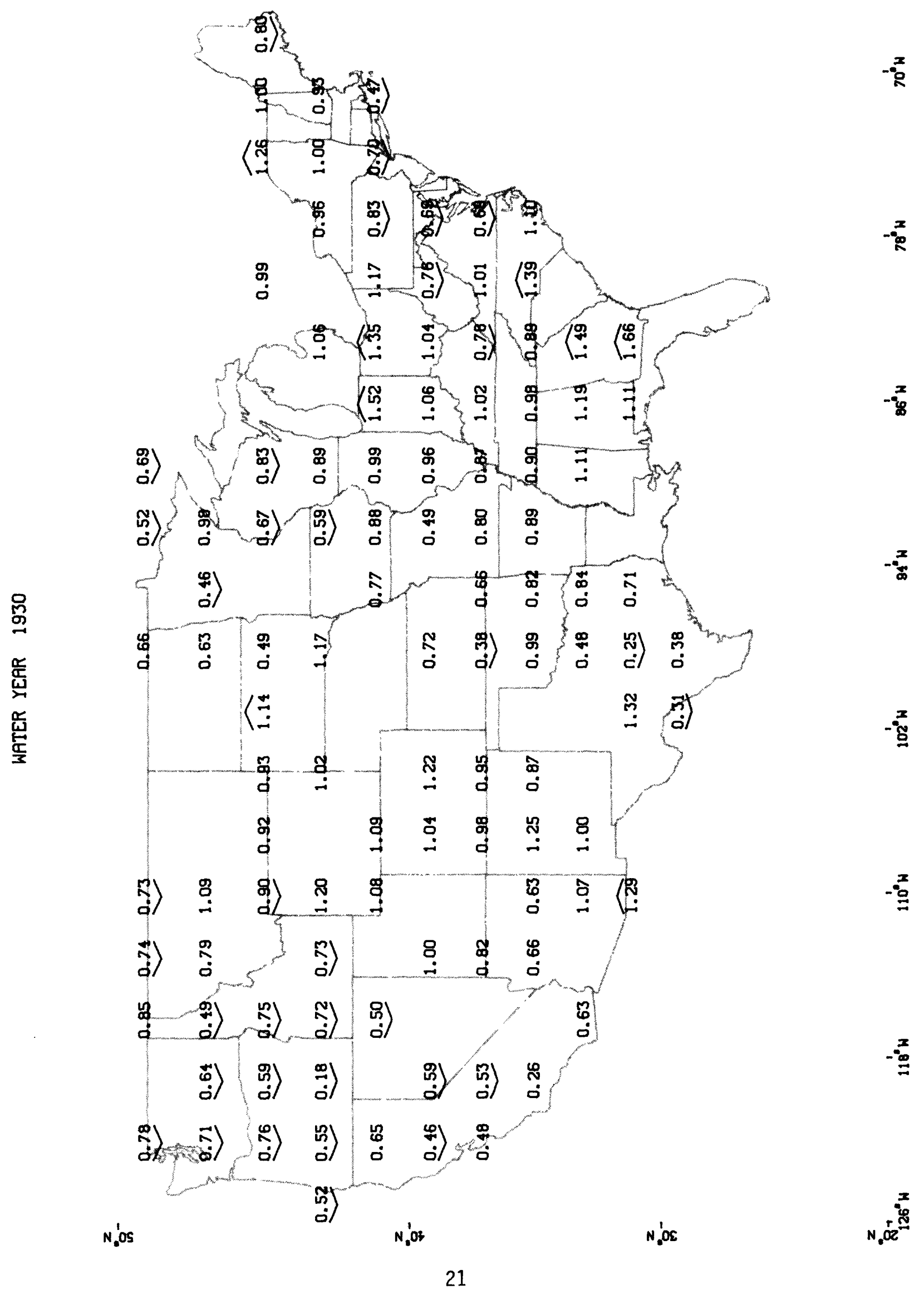




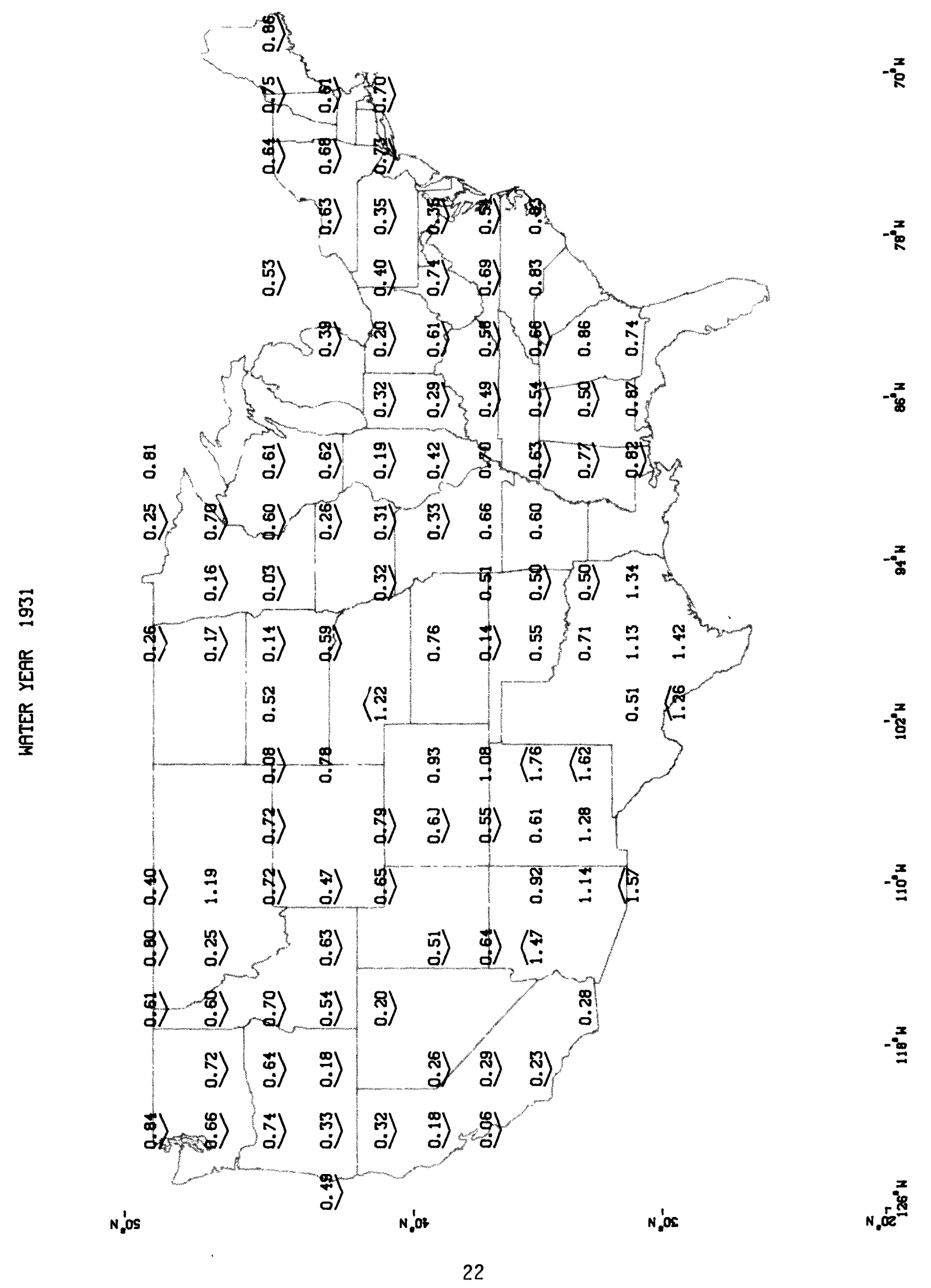




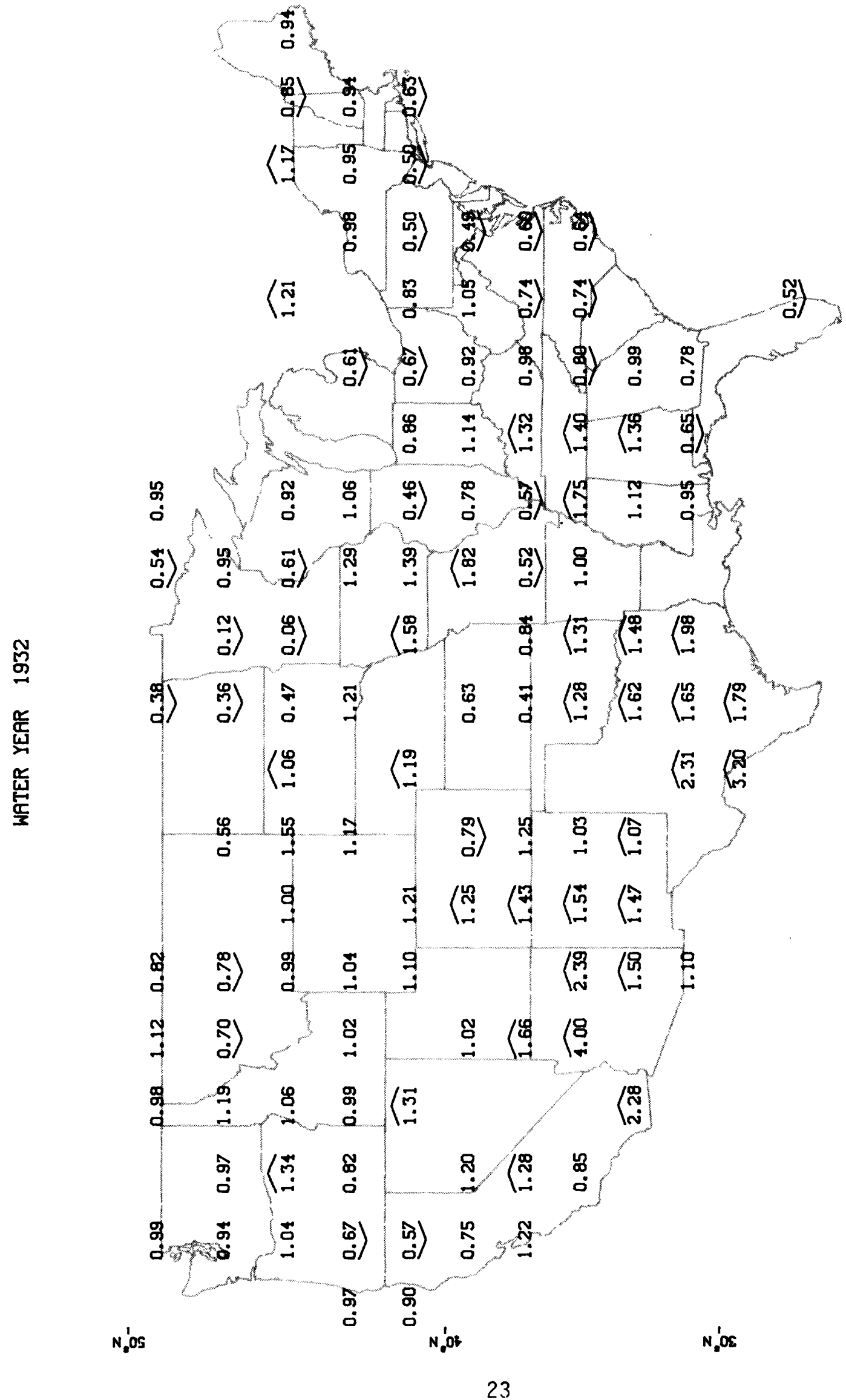

$-\frac{7}{2}$

$-\frac{7}{\infty}$

$-\frac{1}{8}$

$-\frac{7}{\infty}$

-

$-\underset{0}{0}$

$-\frac{1}{ \pm}$ 


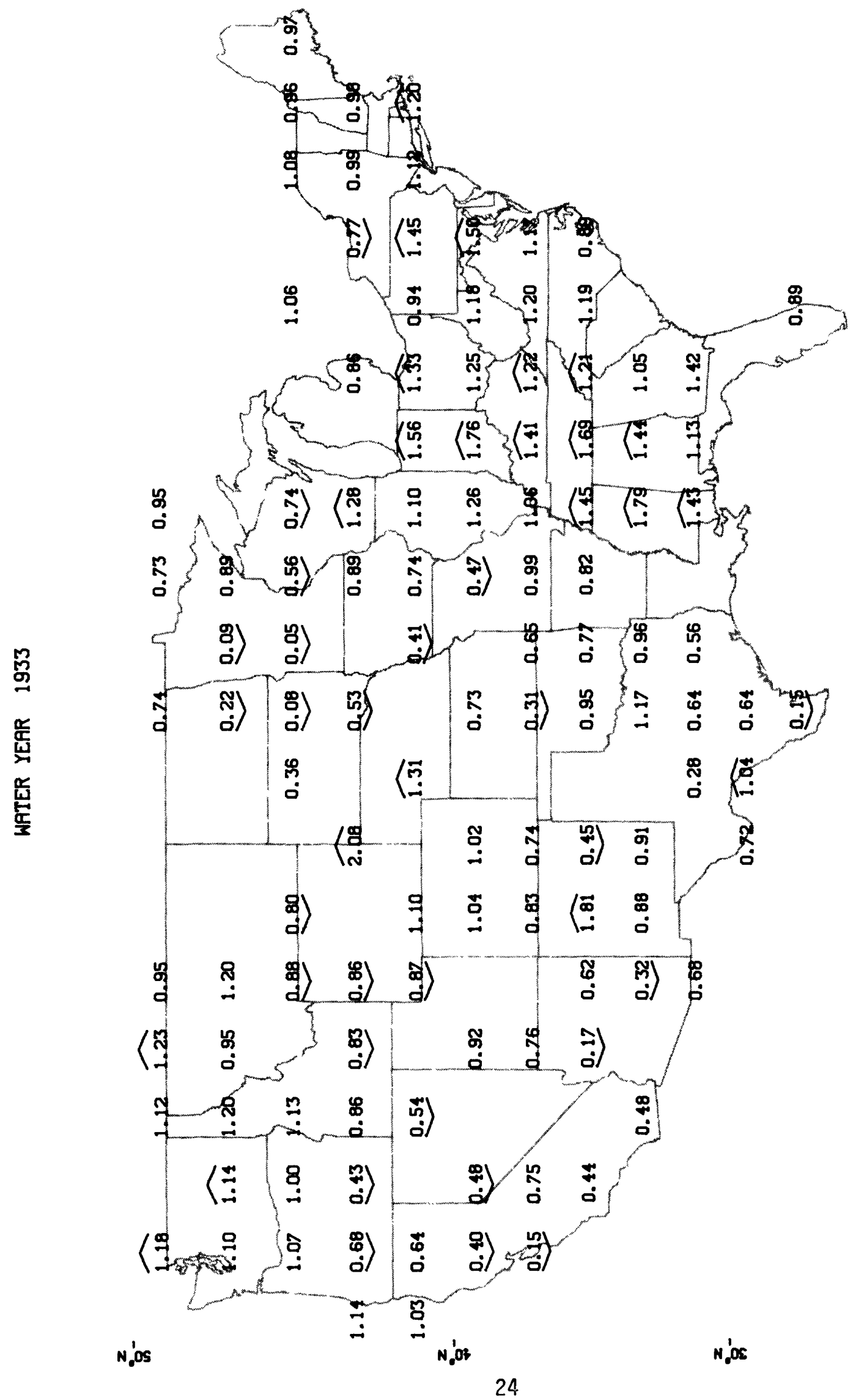

$-\frac{x}{2}$

$-\frac{2}{2}$

$-\frac{x}{8}$

$-\frac{7}{5}$

$-\frac{7}{8}$

$-2$

$\stackrel{-20}{7}$

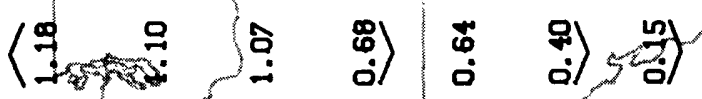




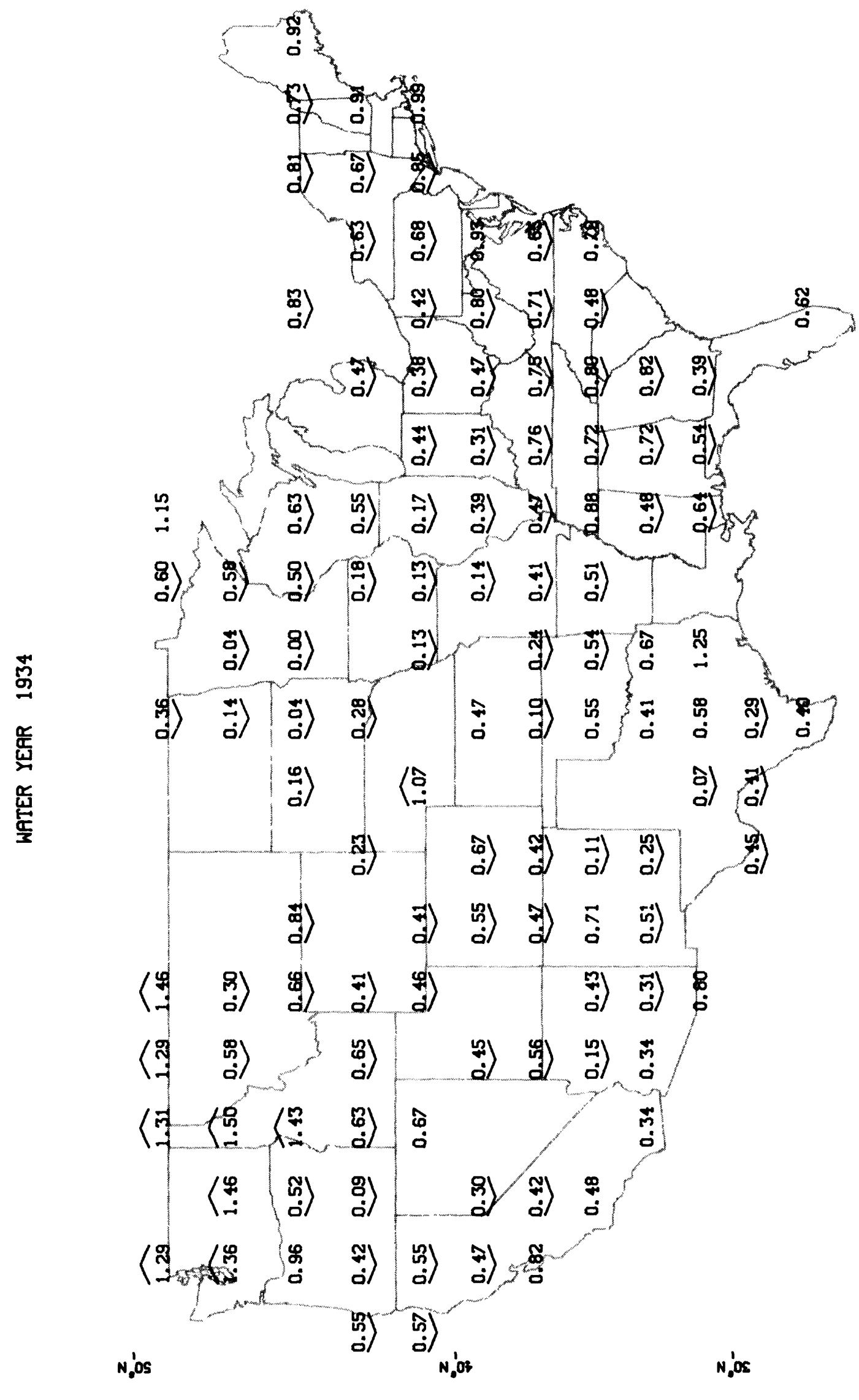




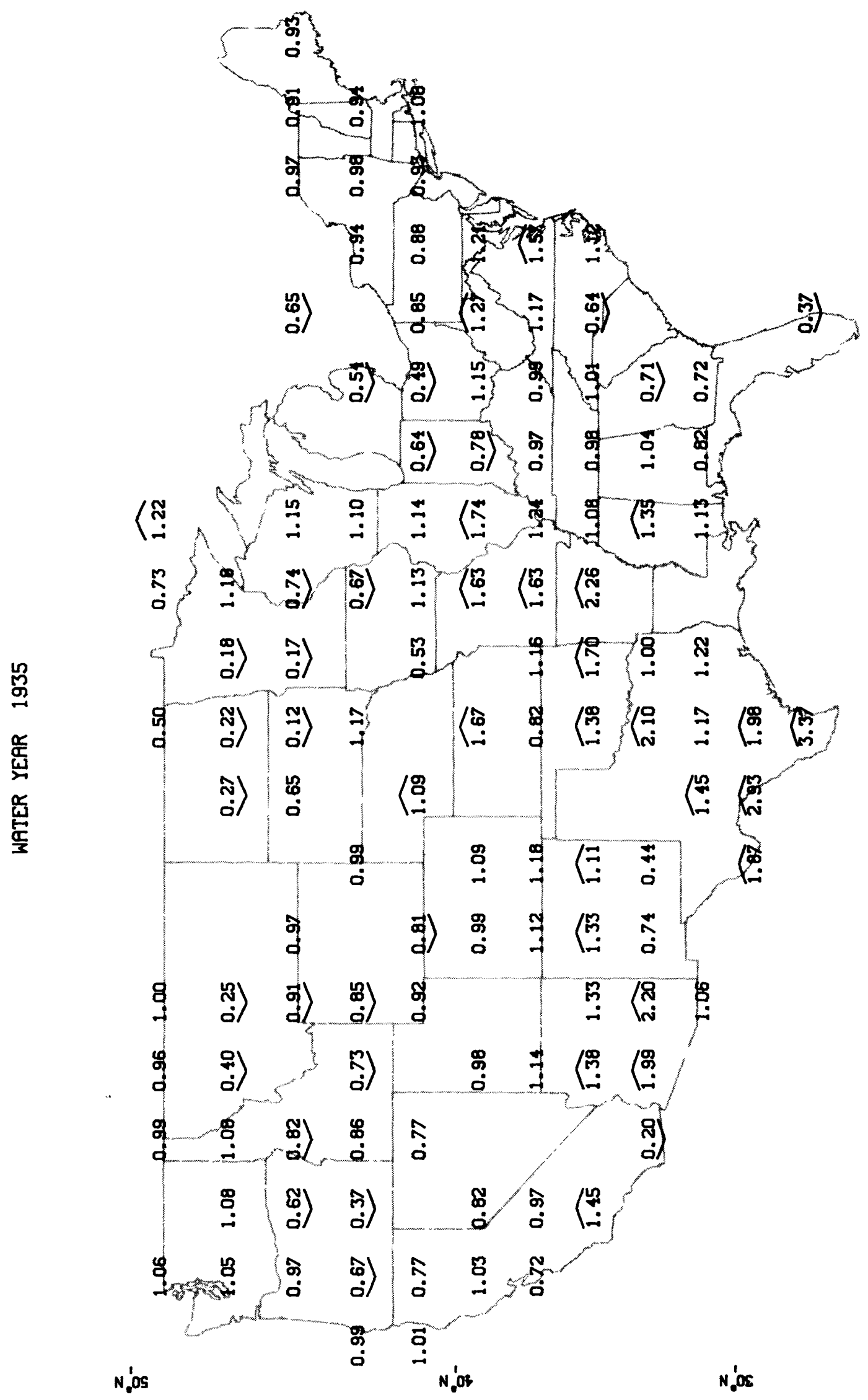

$-\frac{x}{\alpha}$

$-\frac{2}{k}$

$-\frac{7}{8}$

$\frac{7}{8}$

$-\frac{7}{8}$

$-\frac{7}{0}$

$-\frac{3}{2}$ 


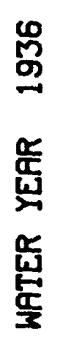

S

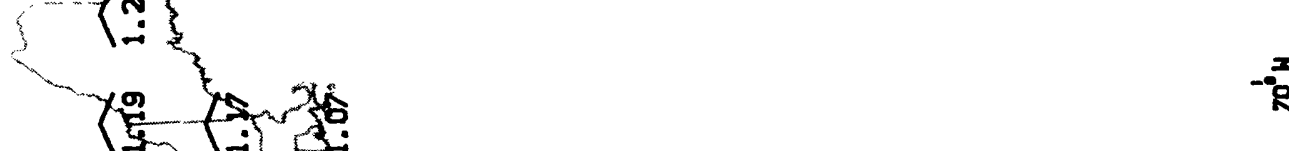

임)

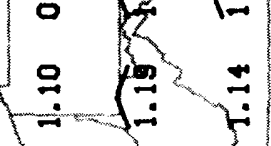

3 .

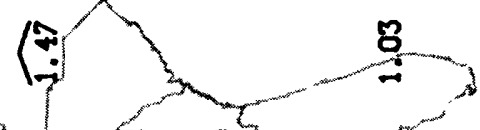

$-\frac{x}{8}$

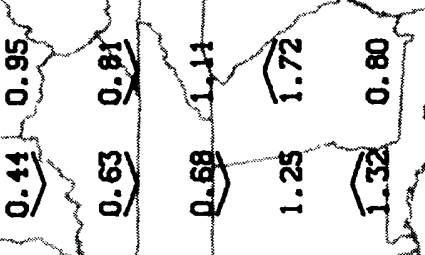

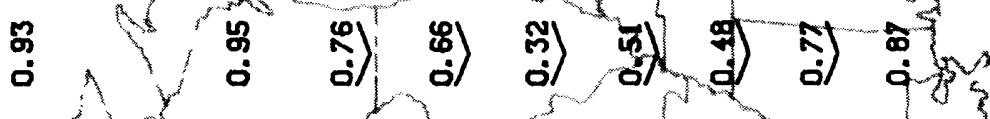

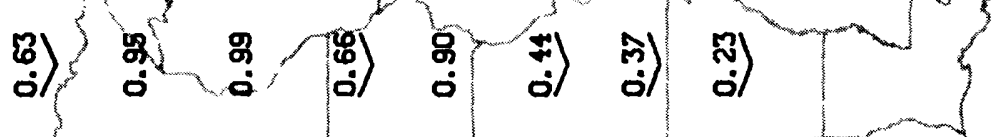

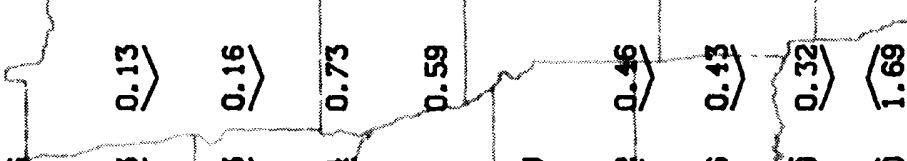

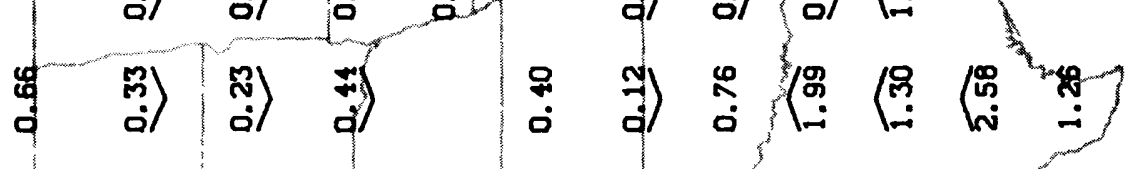

$\frac{x}{2}$

$\frac{7}{8}$

$-\frac{x}{5}$

$-\frac{7}{8}$

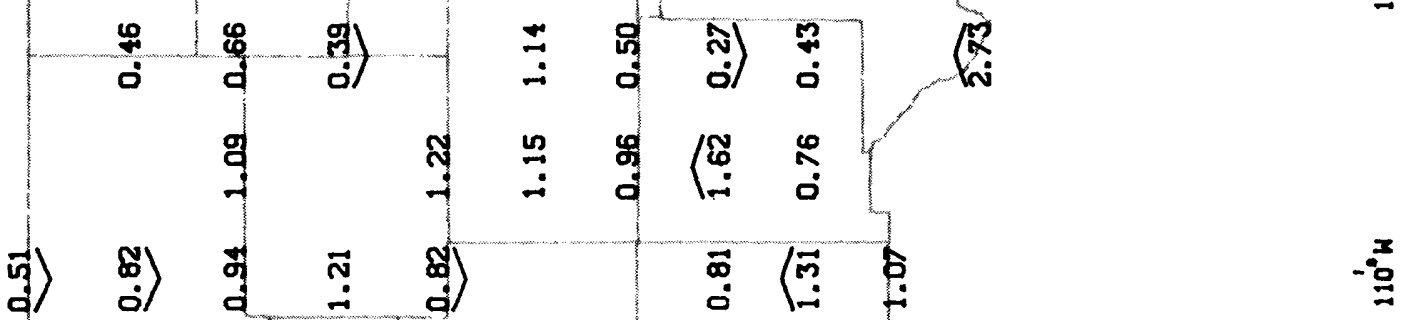

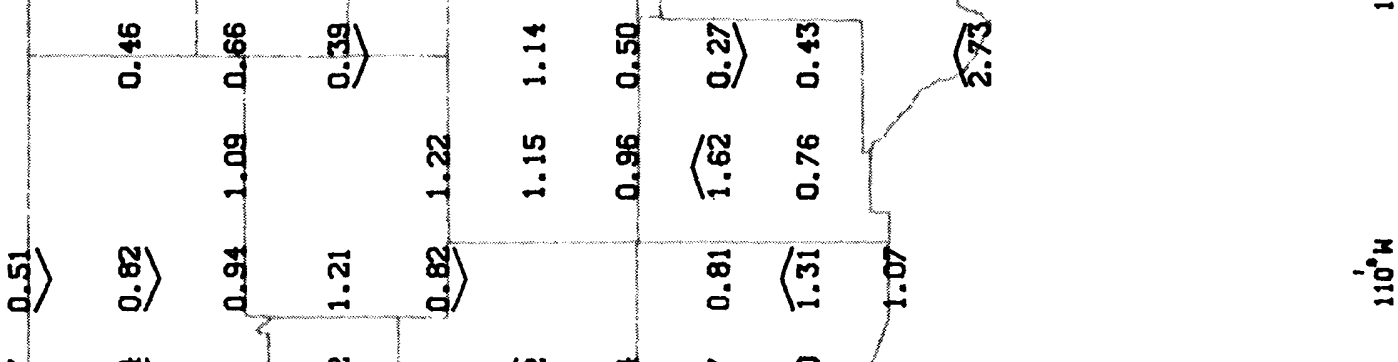

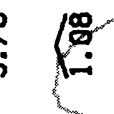

N,OS

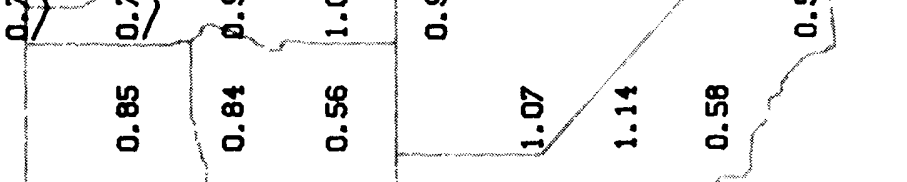

$-\frac{x}{-\infty}$

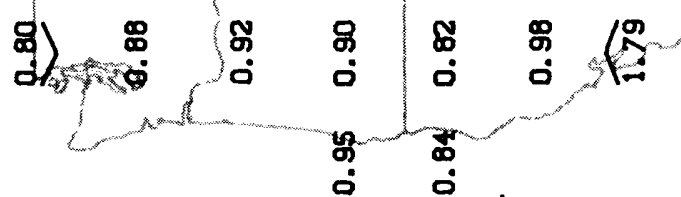

N.08 


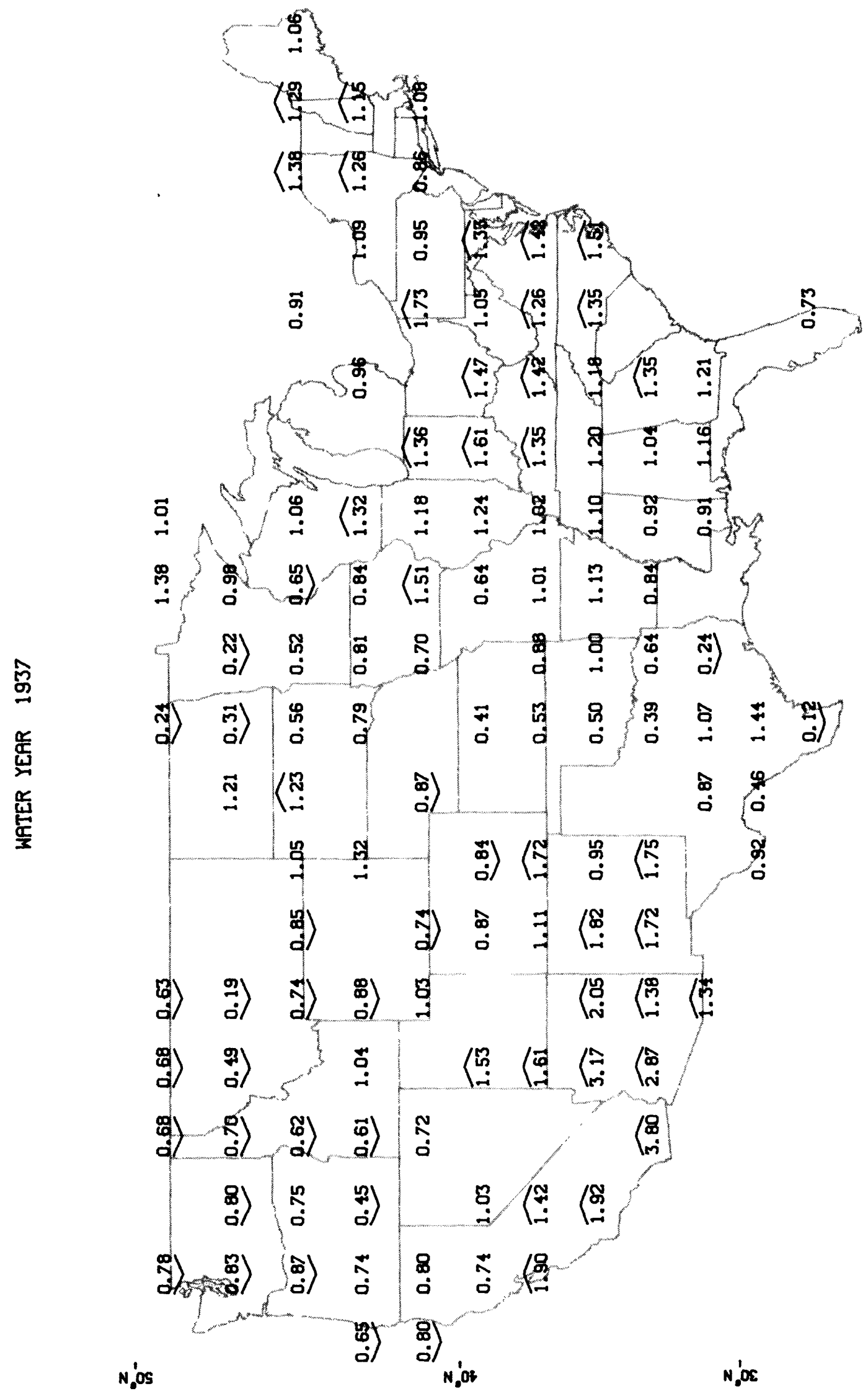




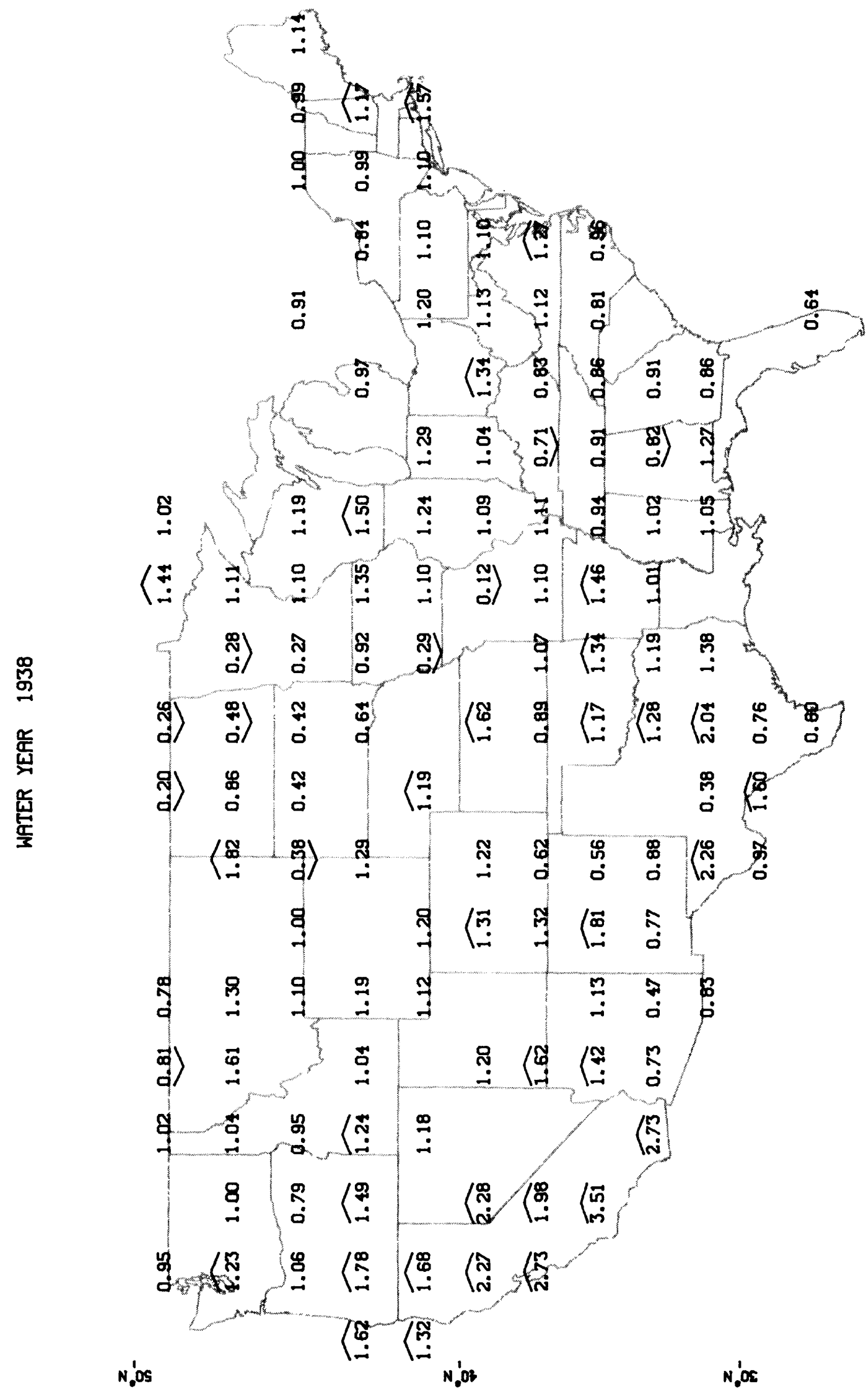

$-\frac{7}{8}$

$-\frac{x}{\infty}$

$-\frac{x}{8}$ 


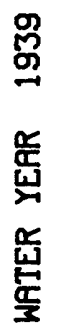

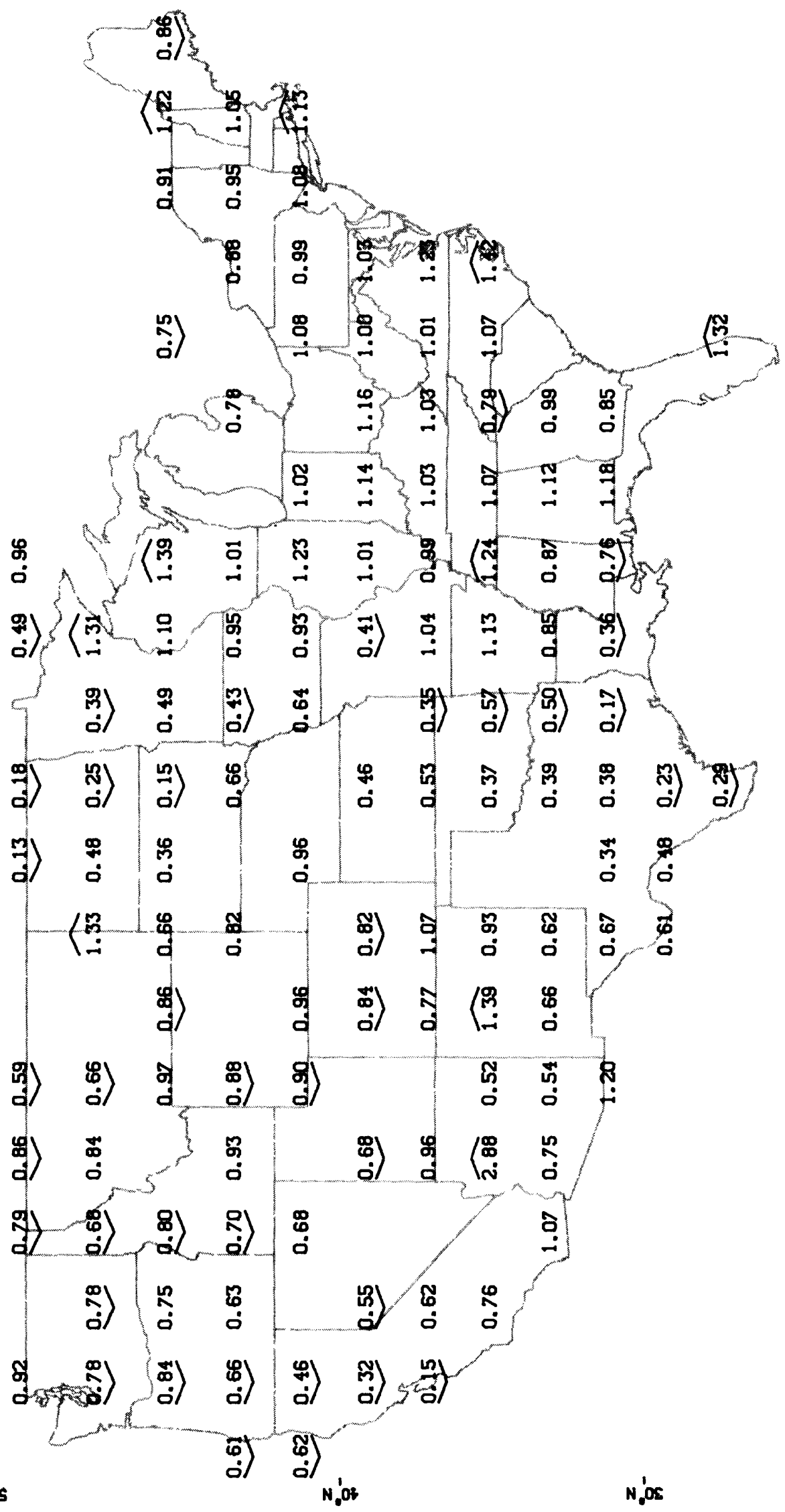




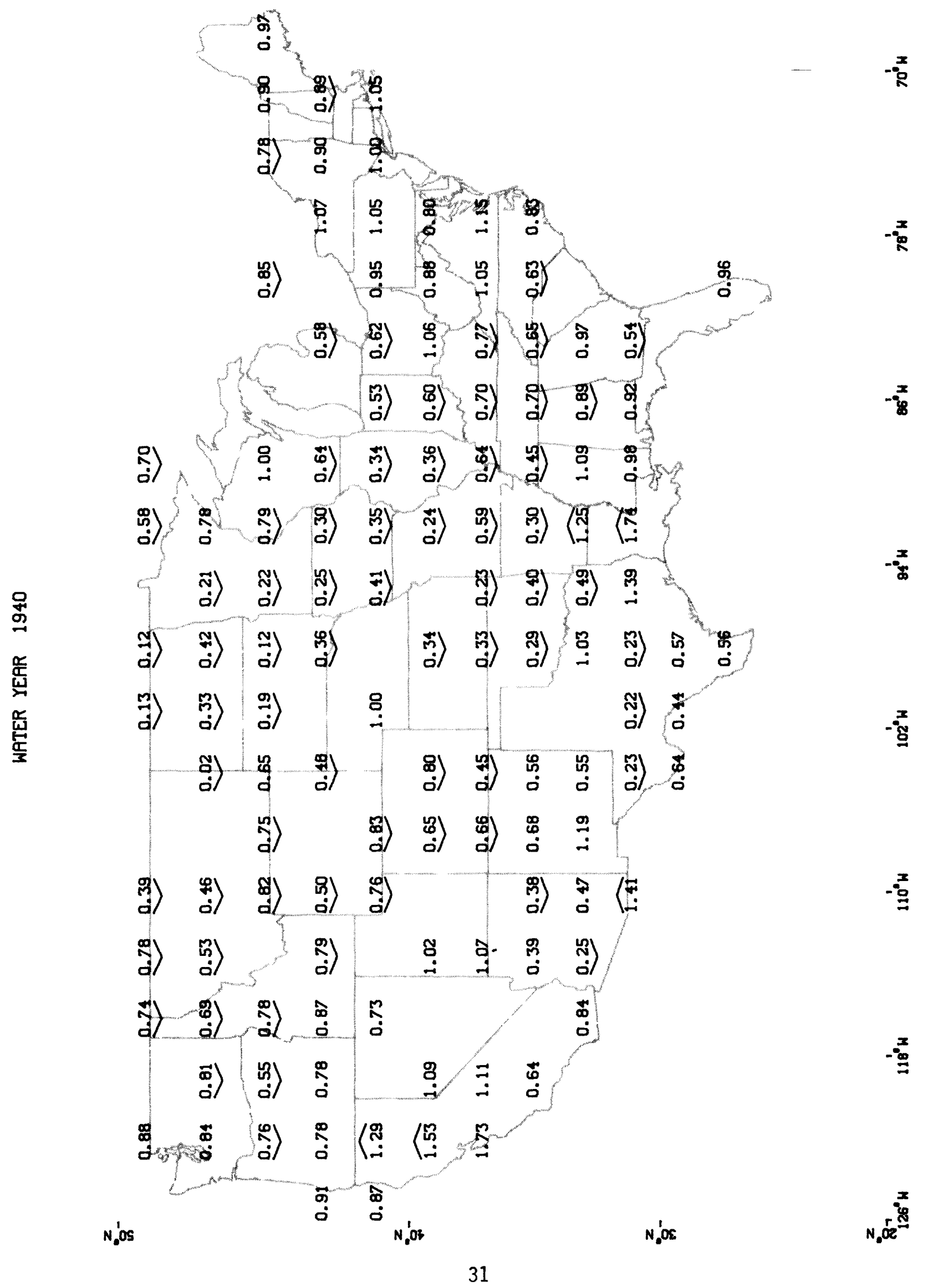




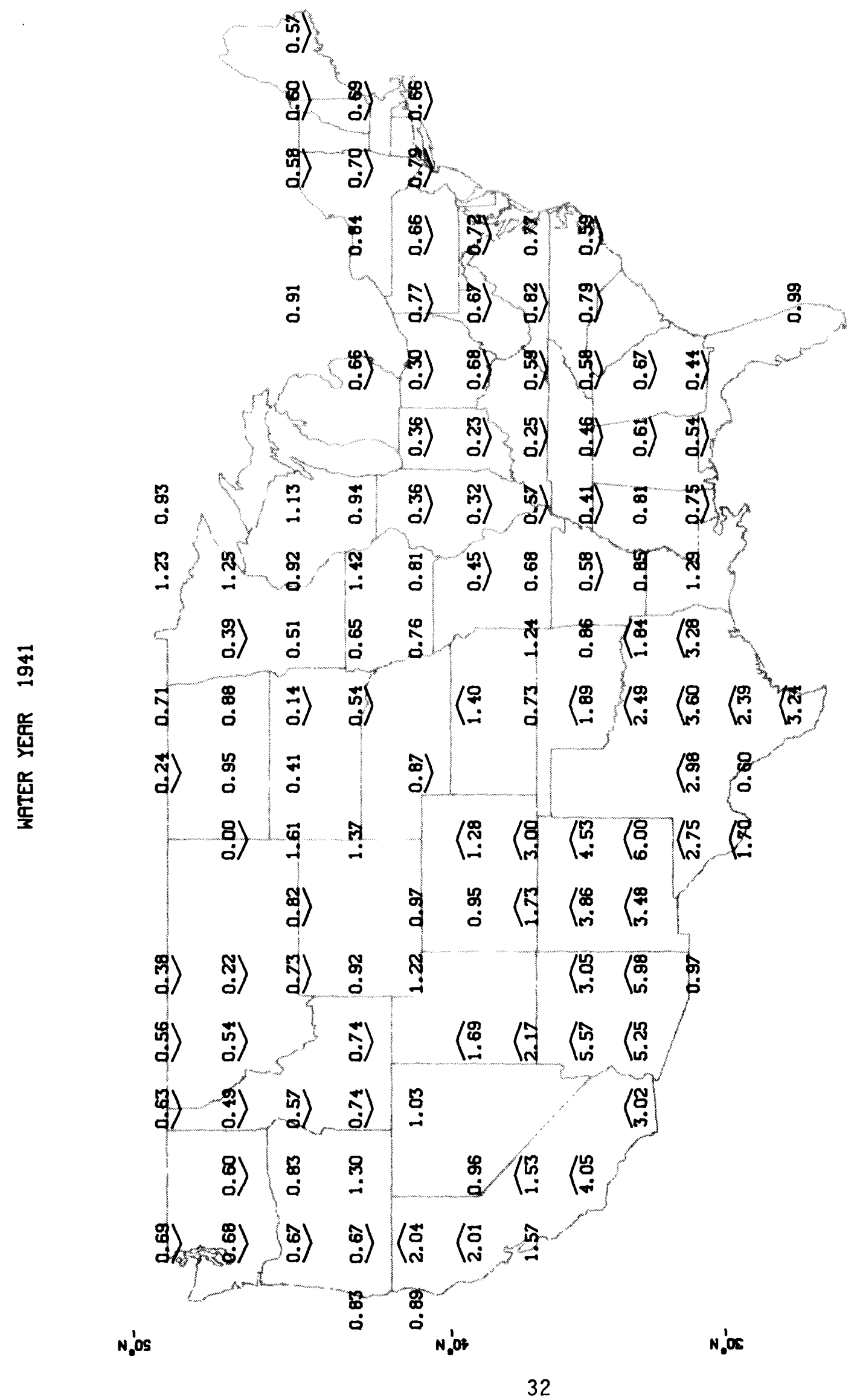




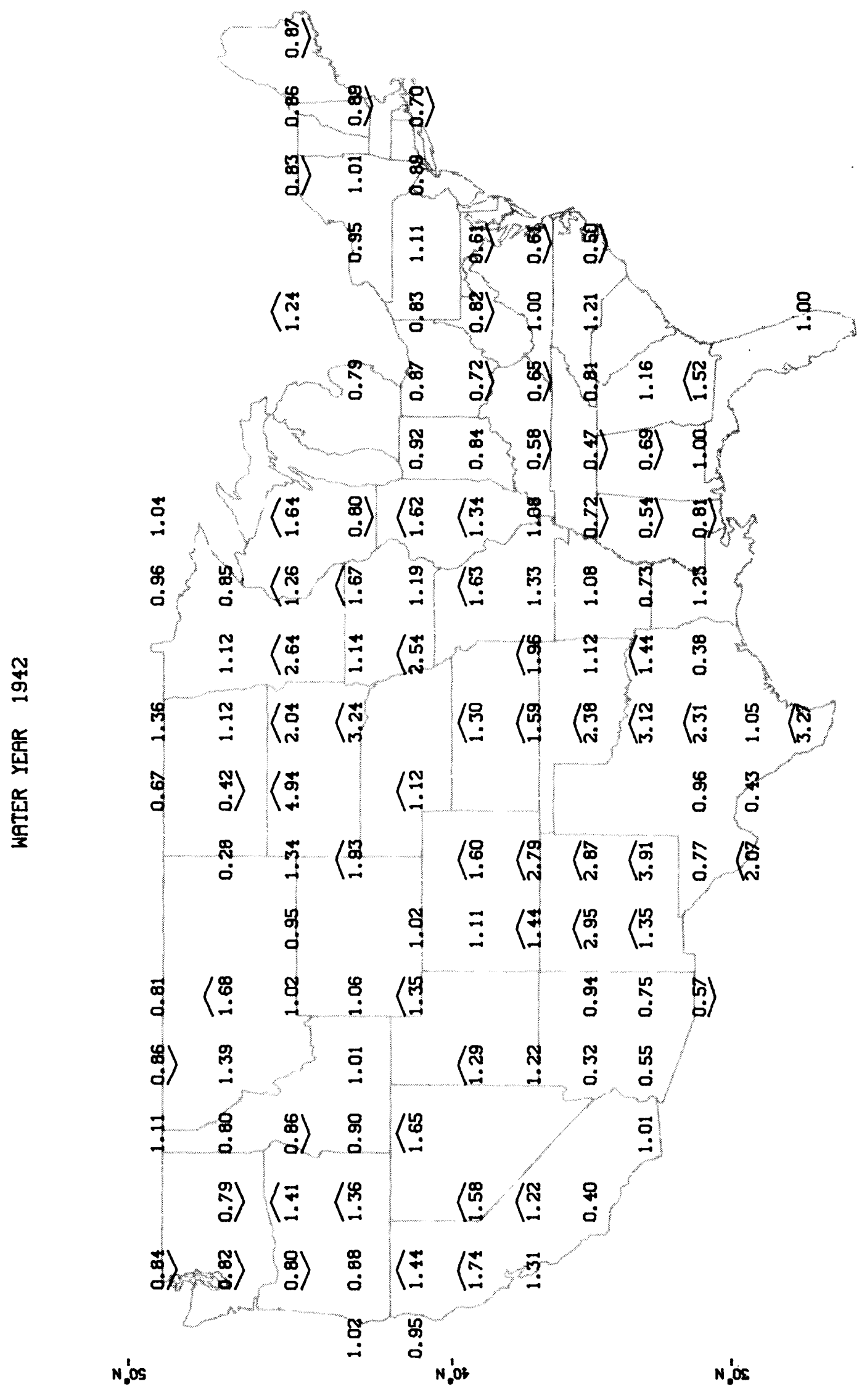




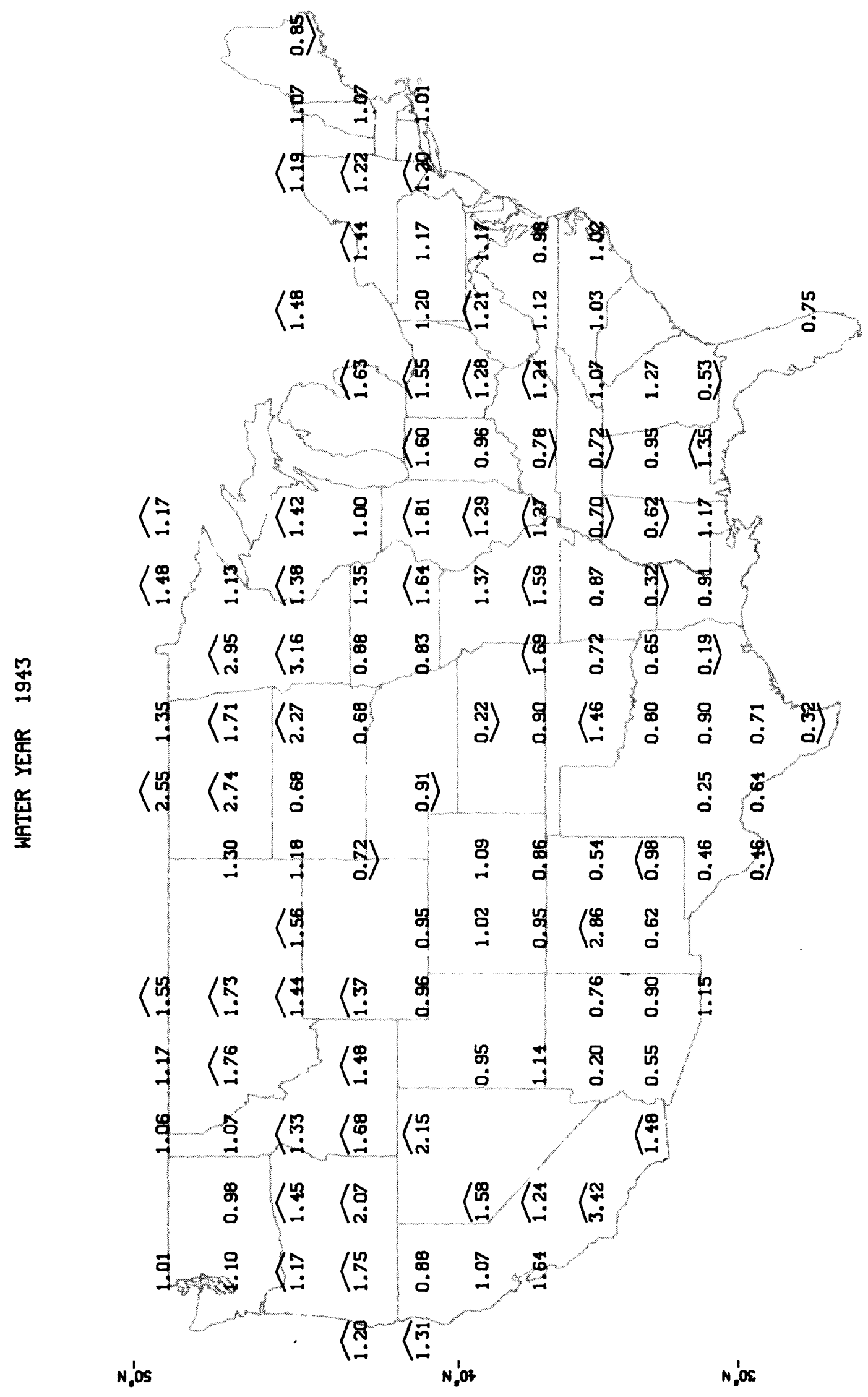

$-\frac{x}{2}$

$-8^{x}$

$-\frac{7}{8}$

$-\frac{2}{8}$

$-\frac{7}{8}$

$-\frac{3}{2}$

$-\stackrel{9}{\Xi}$

${ }^{2}$ 


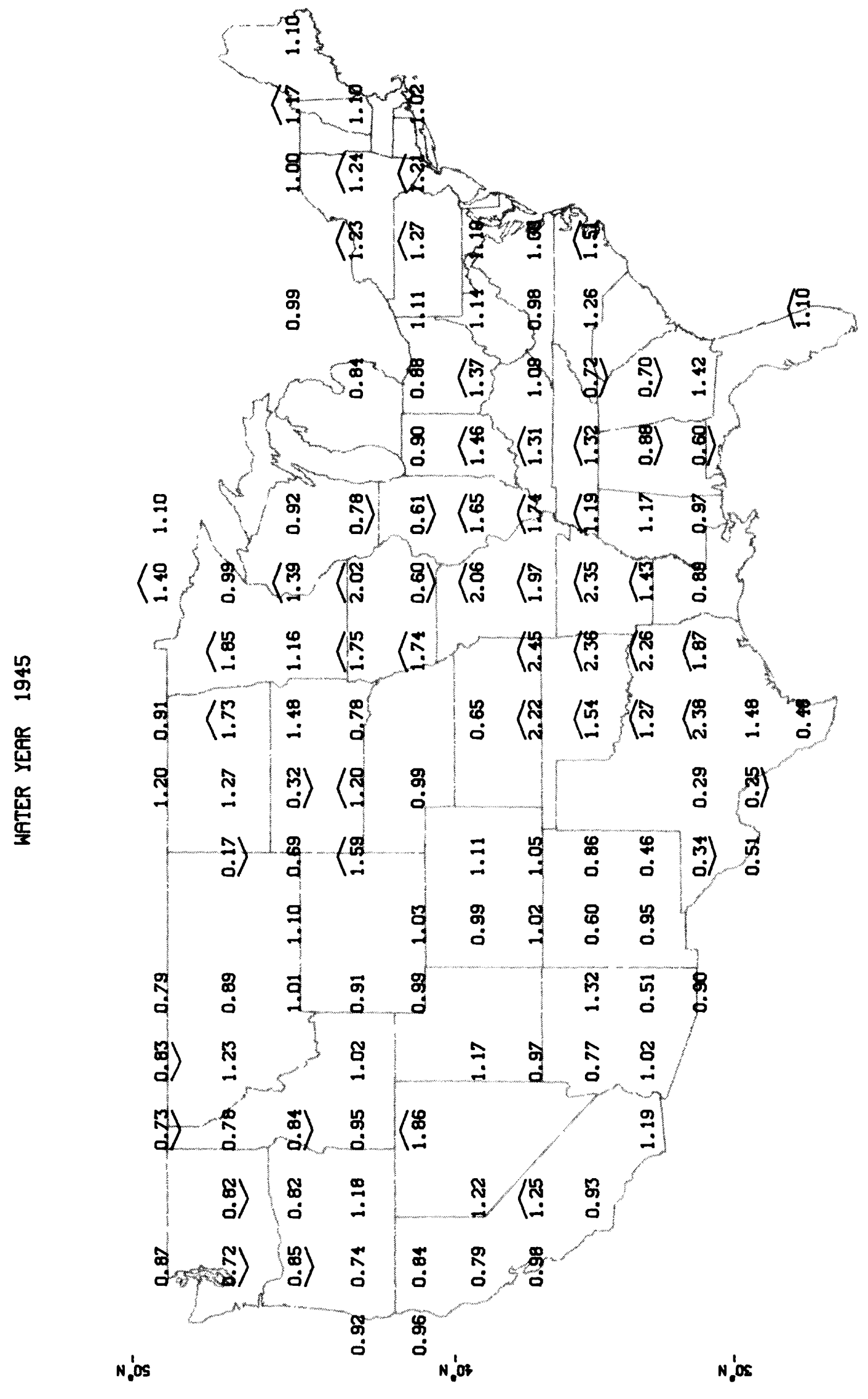

蛋

$-2$

$-\frac{x}{8}$

$-\frac{x}{8}$

$-\frac{x}{5}$

$-\frac{x}{0}$

$-\stackrel{x}{-0}$ 


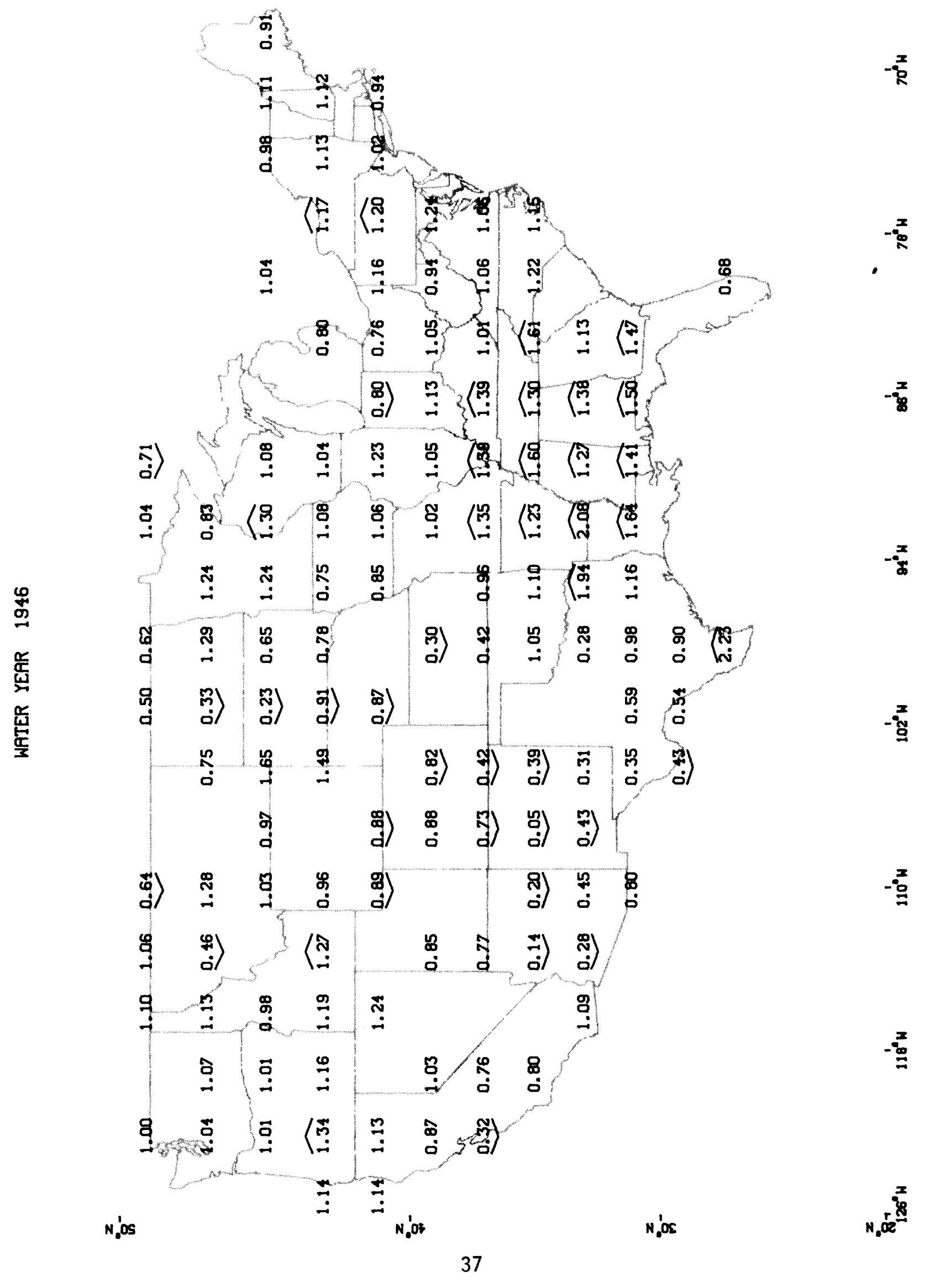




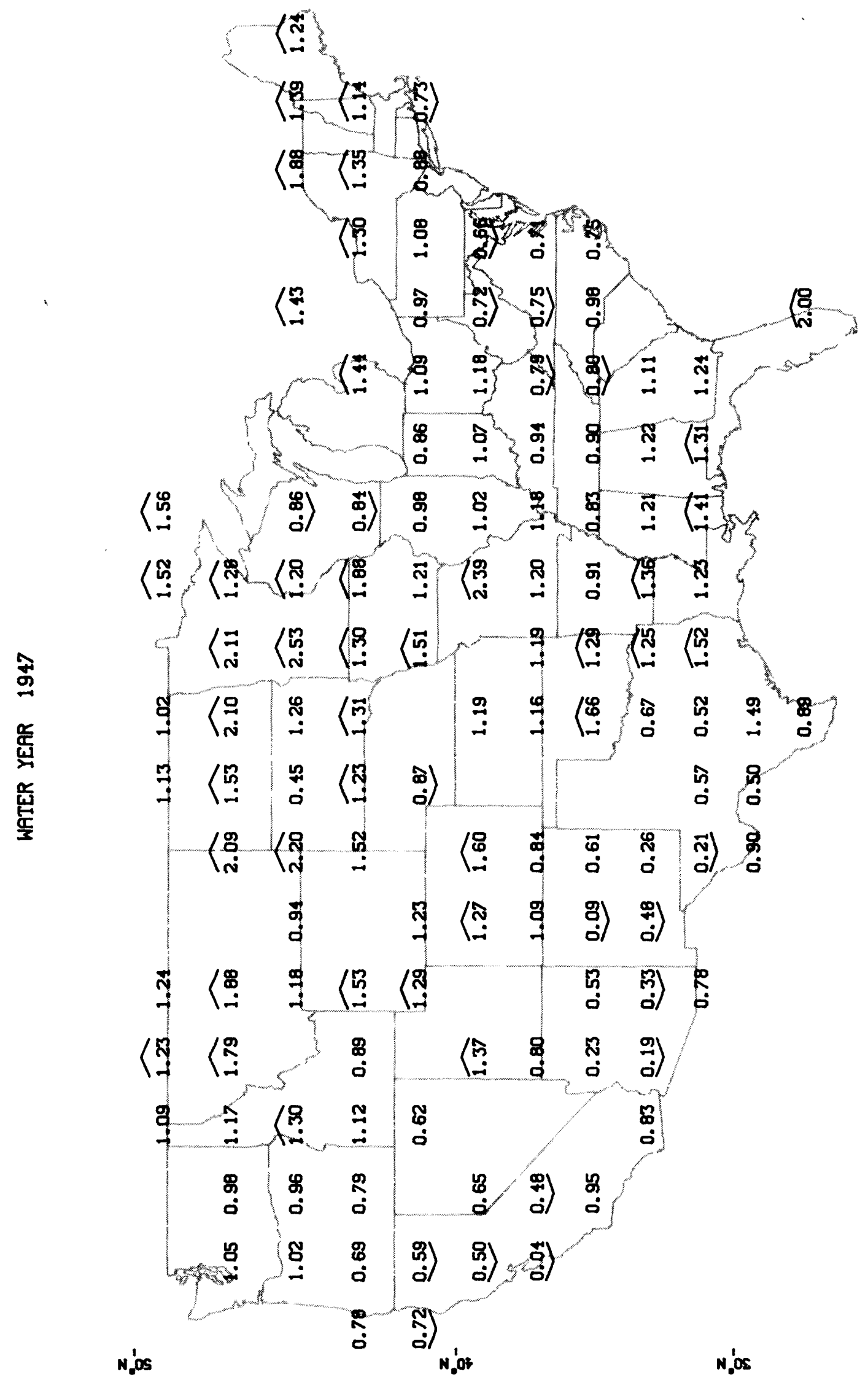

$-\frac{x}{2}$

$-\frac{x}{8}$

$-\frac{7}{8}$

$-\frac{7}{\infty}$

$x$
$-\frac{3}{g}$

$-\stackrel{3}{2}$

$-\underset{7}{ \pm}$ 


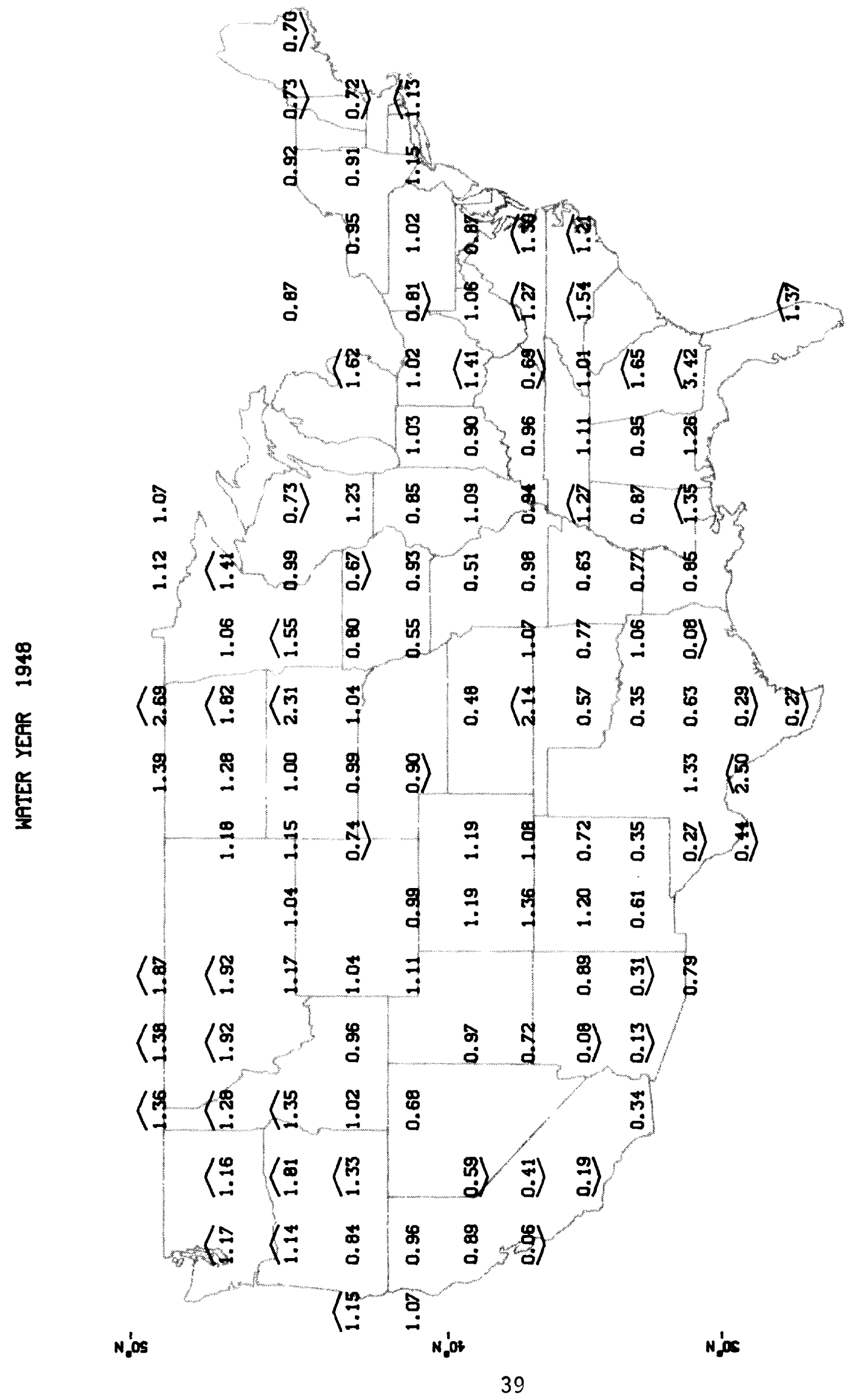

$-\frac{2}{2}$

$-\frac{x}{2}$

$-\frac{7}{8}$

$-\frac{2}{a}$

$-\frac{7}{8}$

$-\frac{x}{0}$

$-2$ 


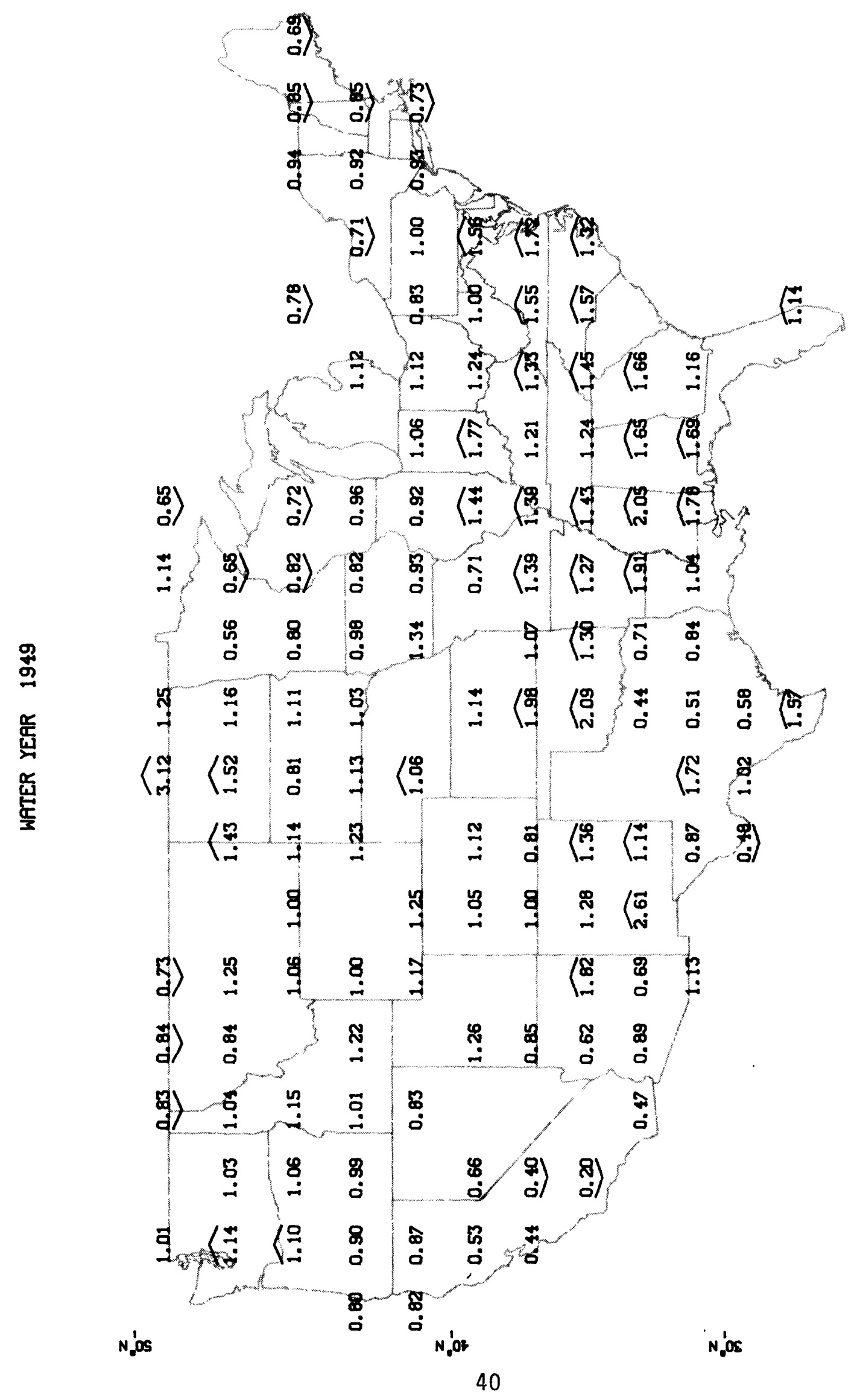




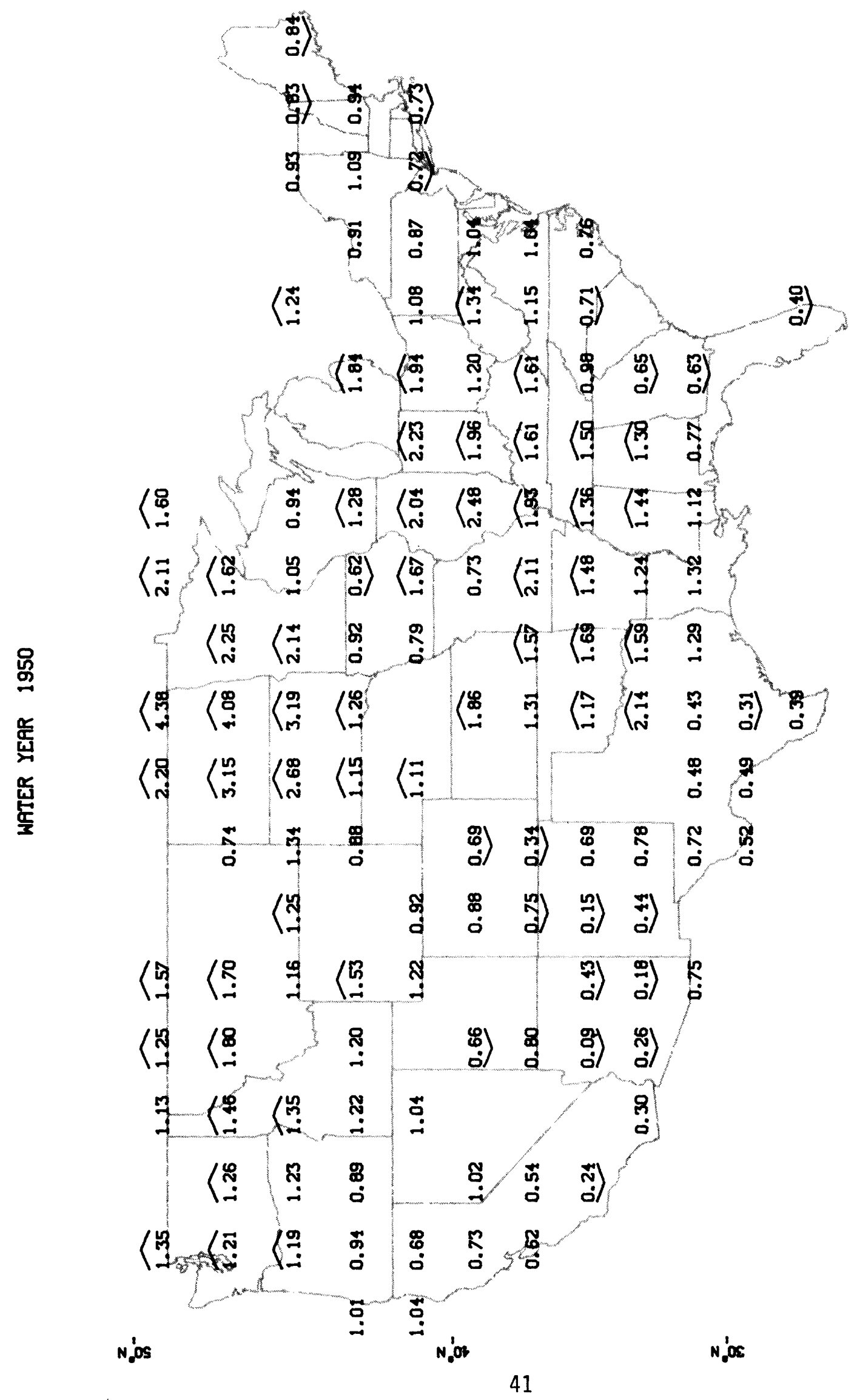

$-\frac{x}{2}$

$-\frac{x}{8}$

$-\frac{7}{8}$

$-\frac{x}{8}$

$-\frac{x}{5}$

$-\stackrel{3}{3}$

$-2$

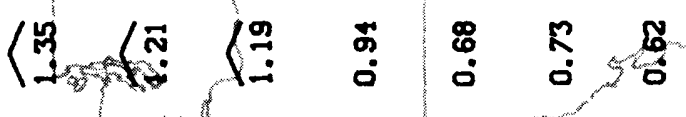

Nos 


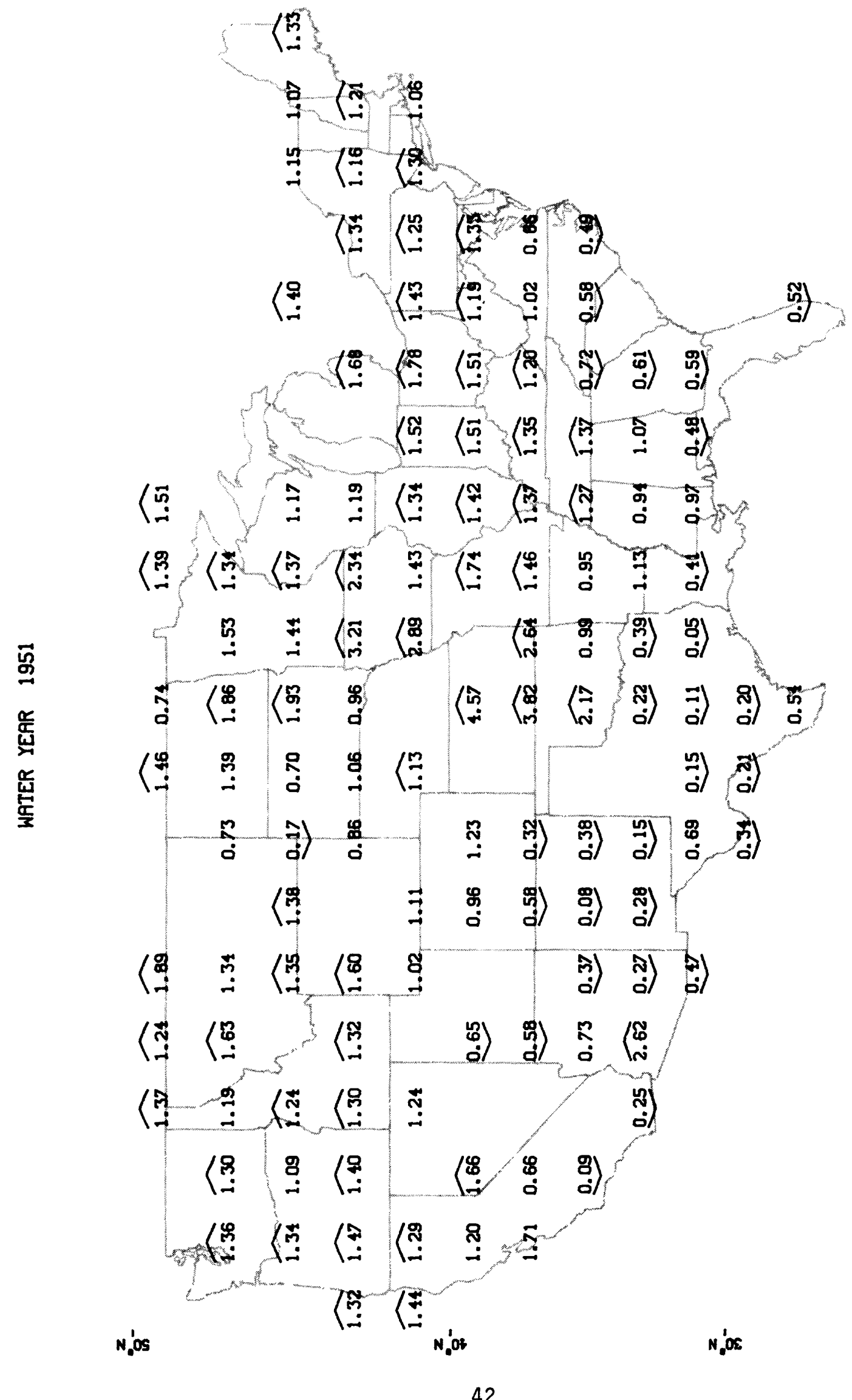

$-\frac{7}{2}$

$-8$

$-8$

$\frac{x}{2}$

-

$-\stackrel{x}{-0}$

$-\stackrel{x}{=}$ 


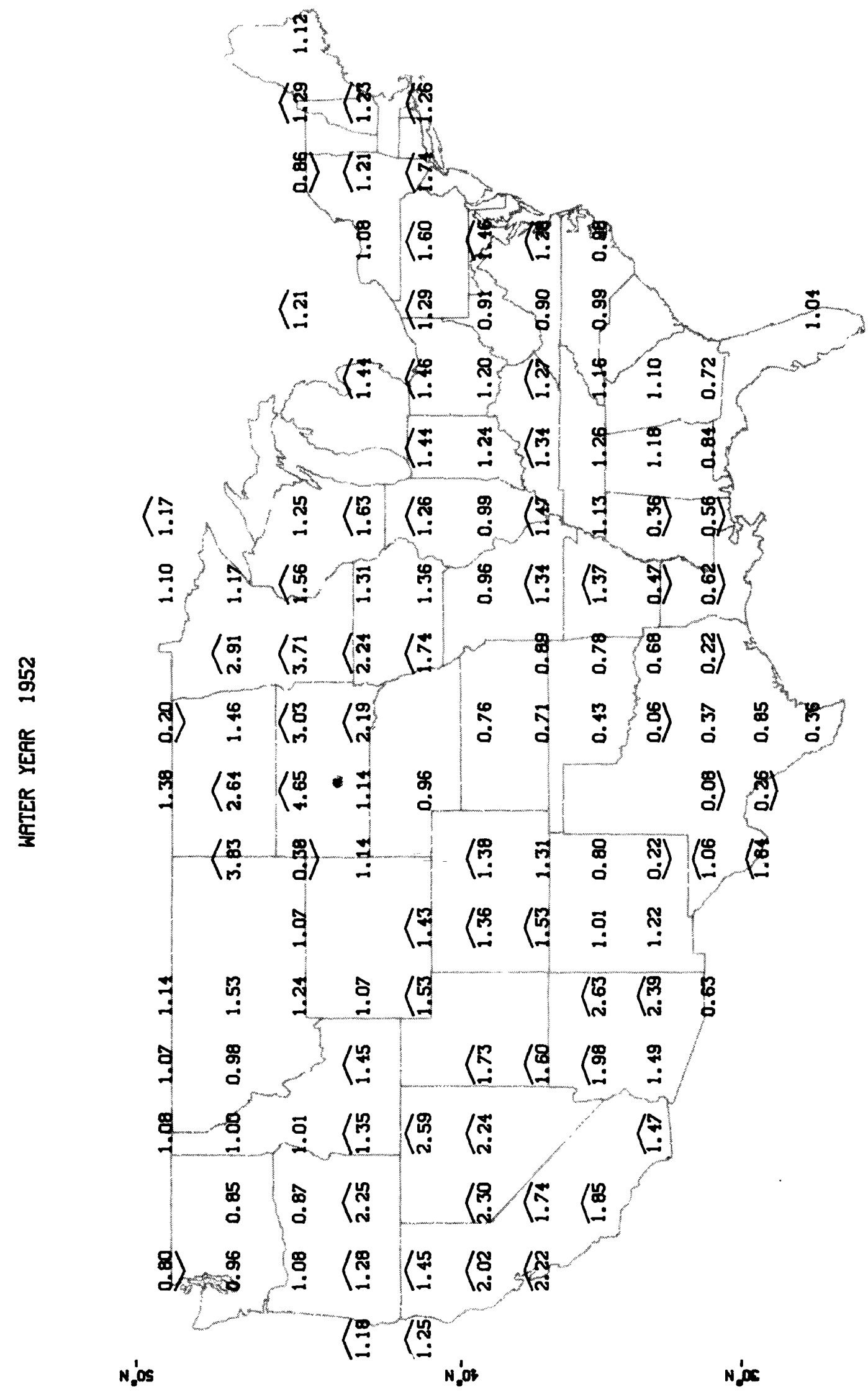

$-\frac{x}{2}$

$-\frac{x}{2}$

$-\frac{x}{8}$

$-\frac{x}{5}$

$-\frac{7}{8}$

$-\frac{7}{2}$

$-2$ 


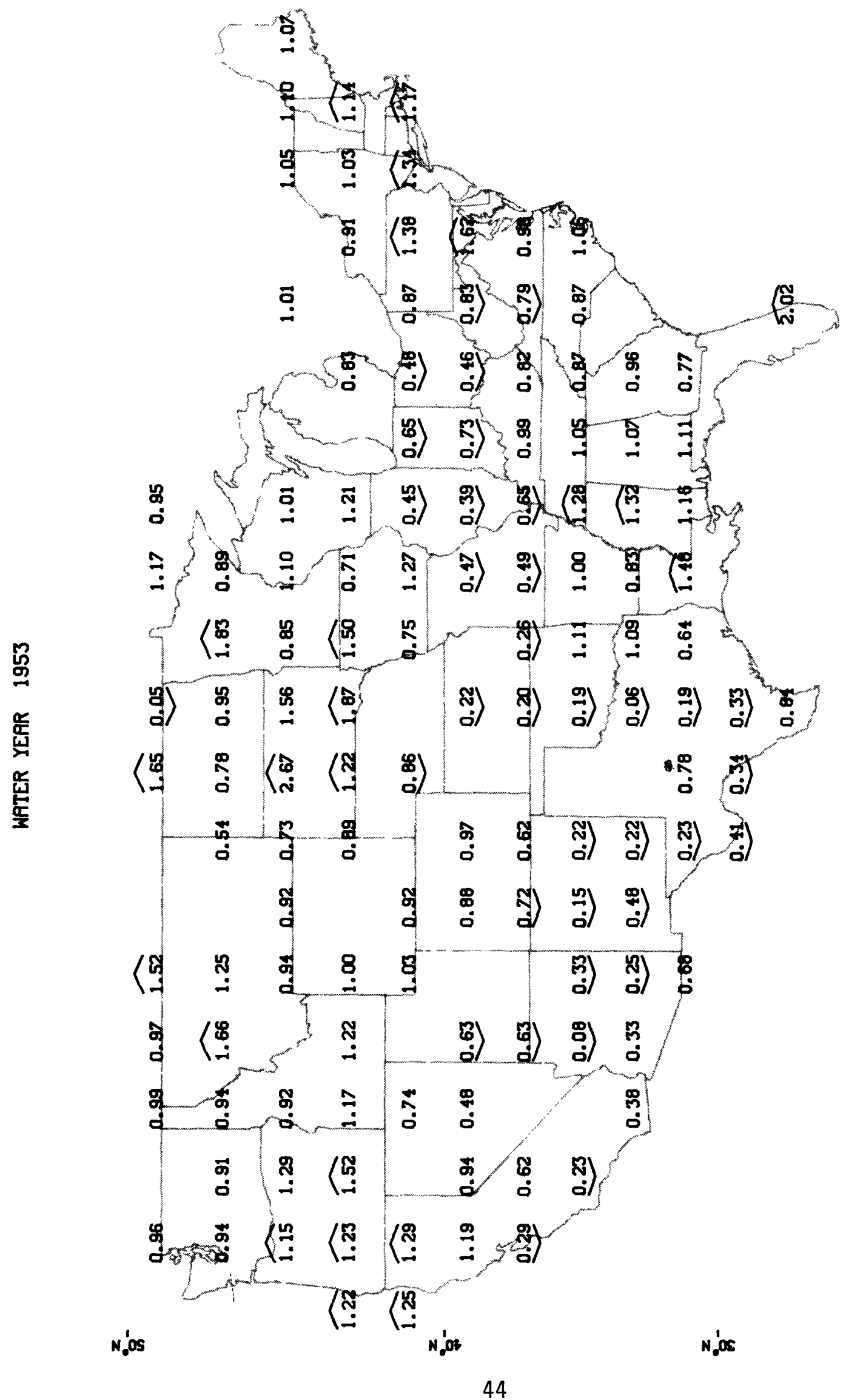




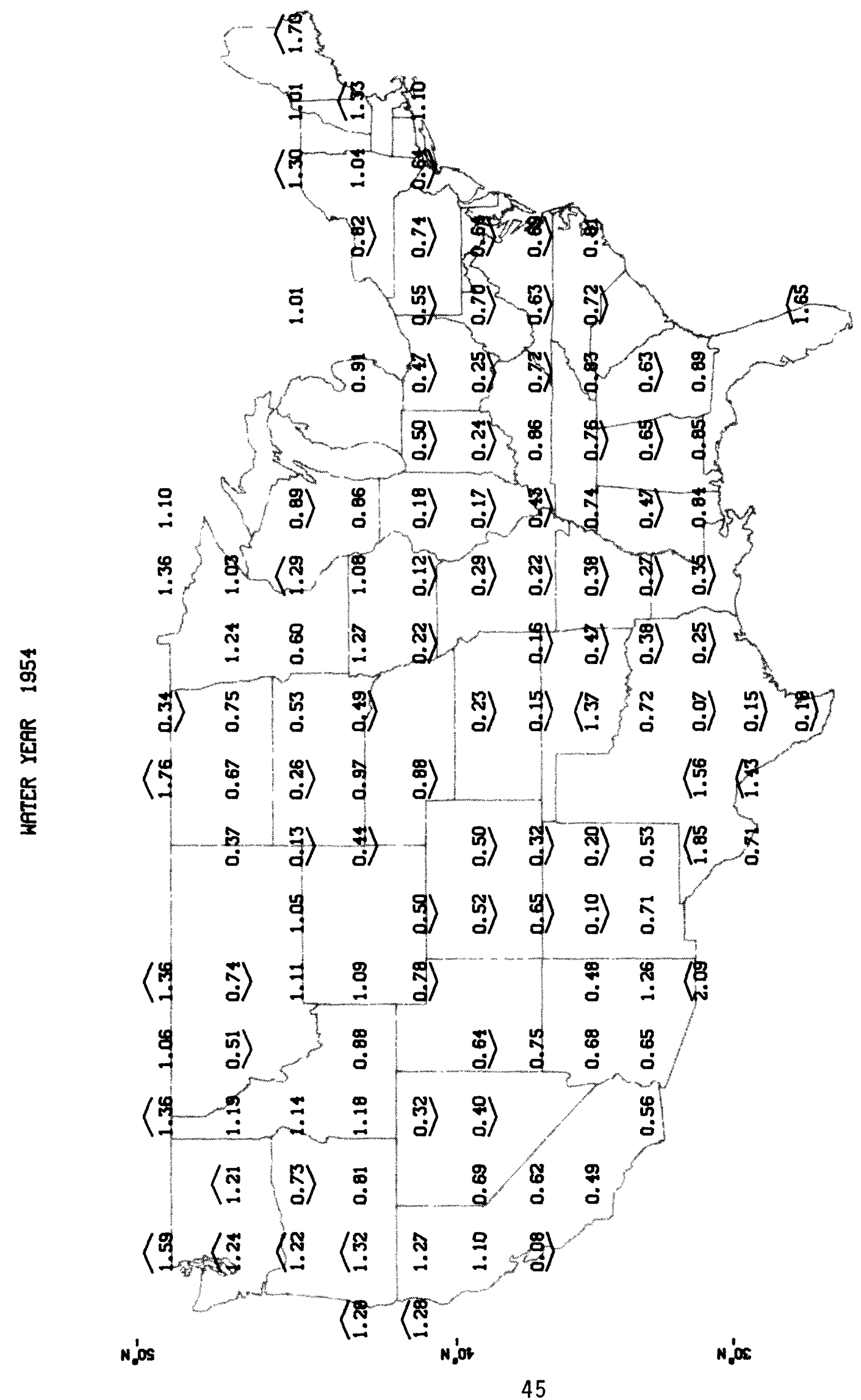

$-\frac{7}{8}$

$-8$

$-\frac{x}{d}$

$-\frac{7}{8}$

$-\underline{x}$

$-\frac{x}{=}$ 


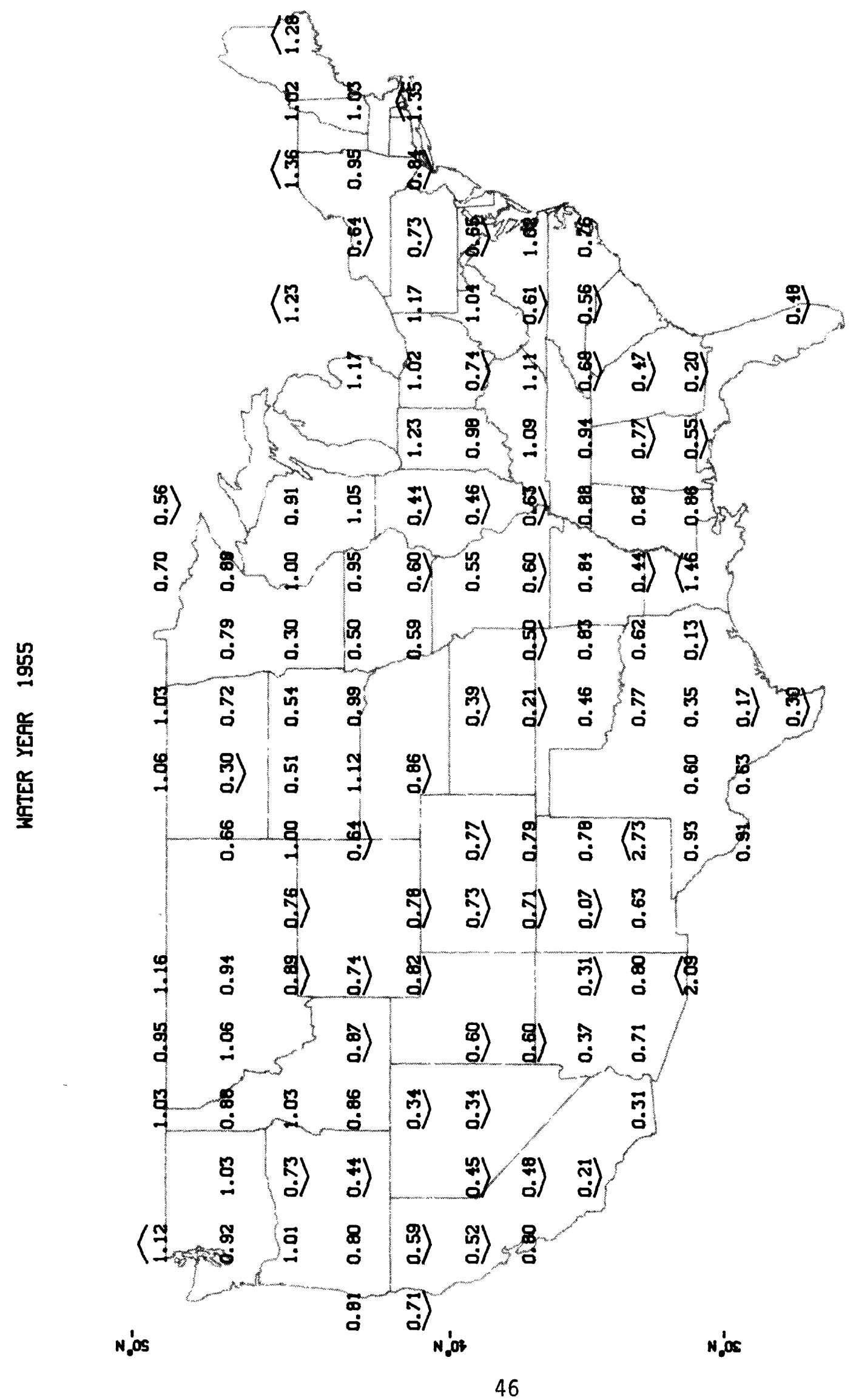

$-\frac{x}{R}$

$-\frac{x}{8}$

$-\frac{x}{8}$

$-\frac{x}{2}$

$-\frac{x}{8}$

$-\frac{7}{2}$

$-\stackrel{x}{=}$ 


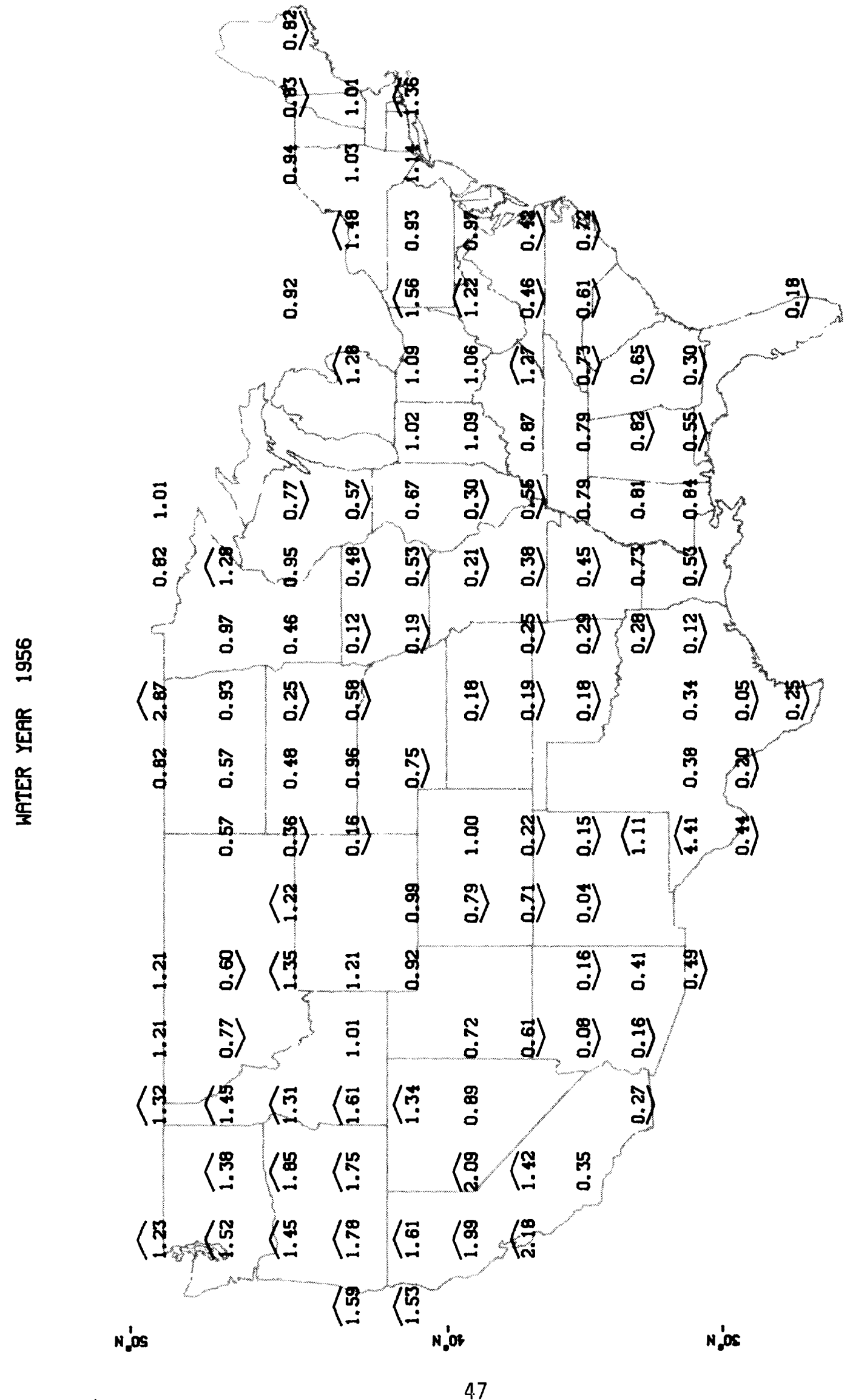

$-\frac{7}{R}$

$-\frac{7}{8}$

$-\frac{7}{8}$

$-\frac{7}{\infty}$

$-\frac{7}{8}$

$-\frac{2}{0}$

$-\stackrel{x}{\varrho}$ 


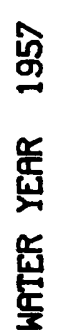

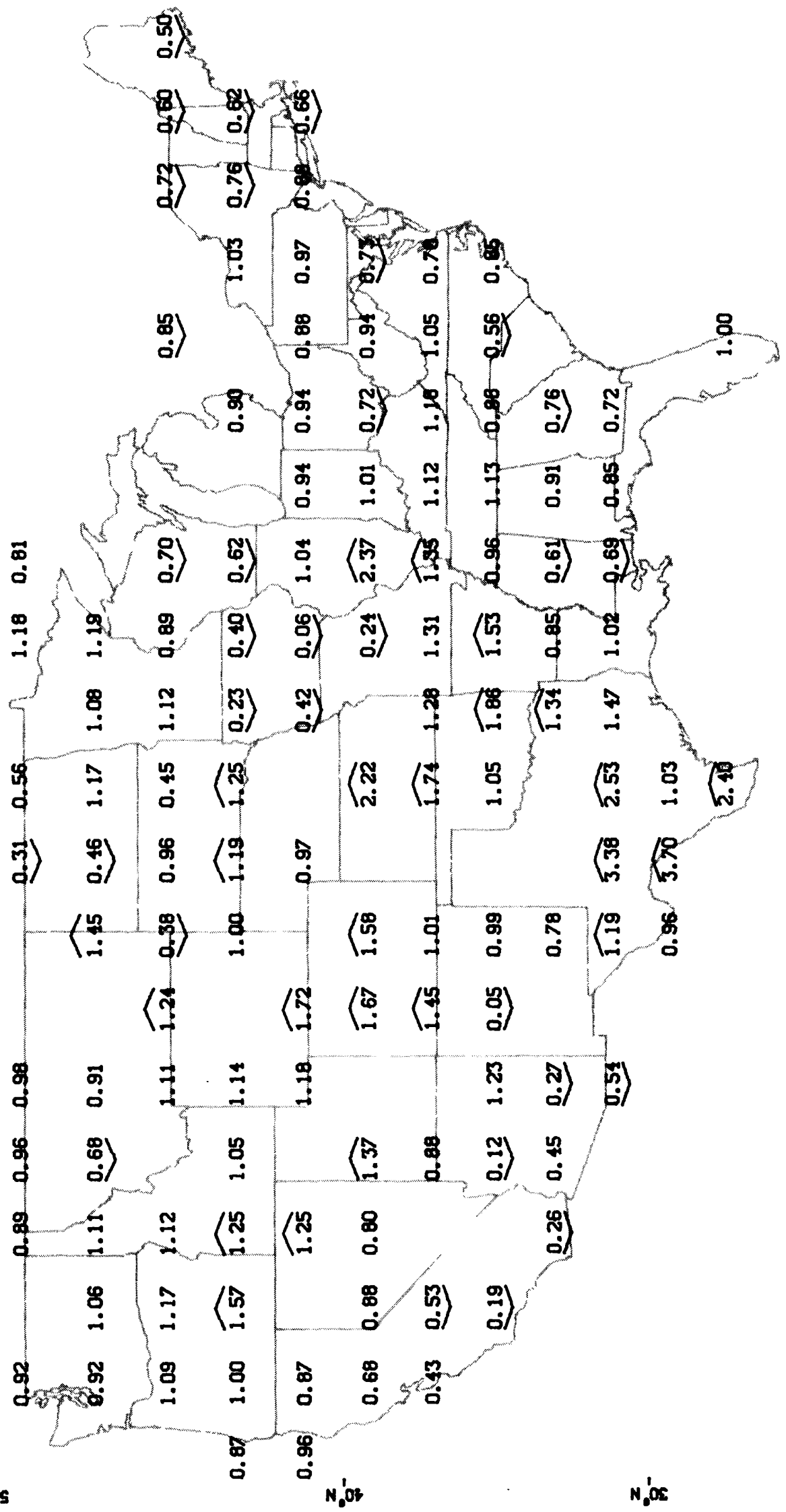




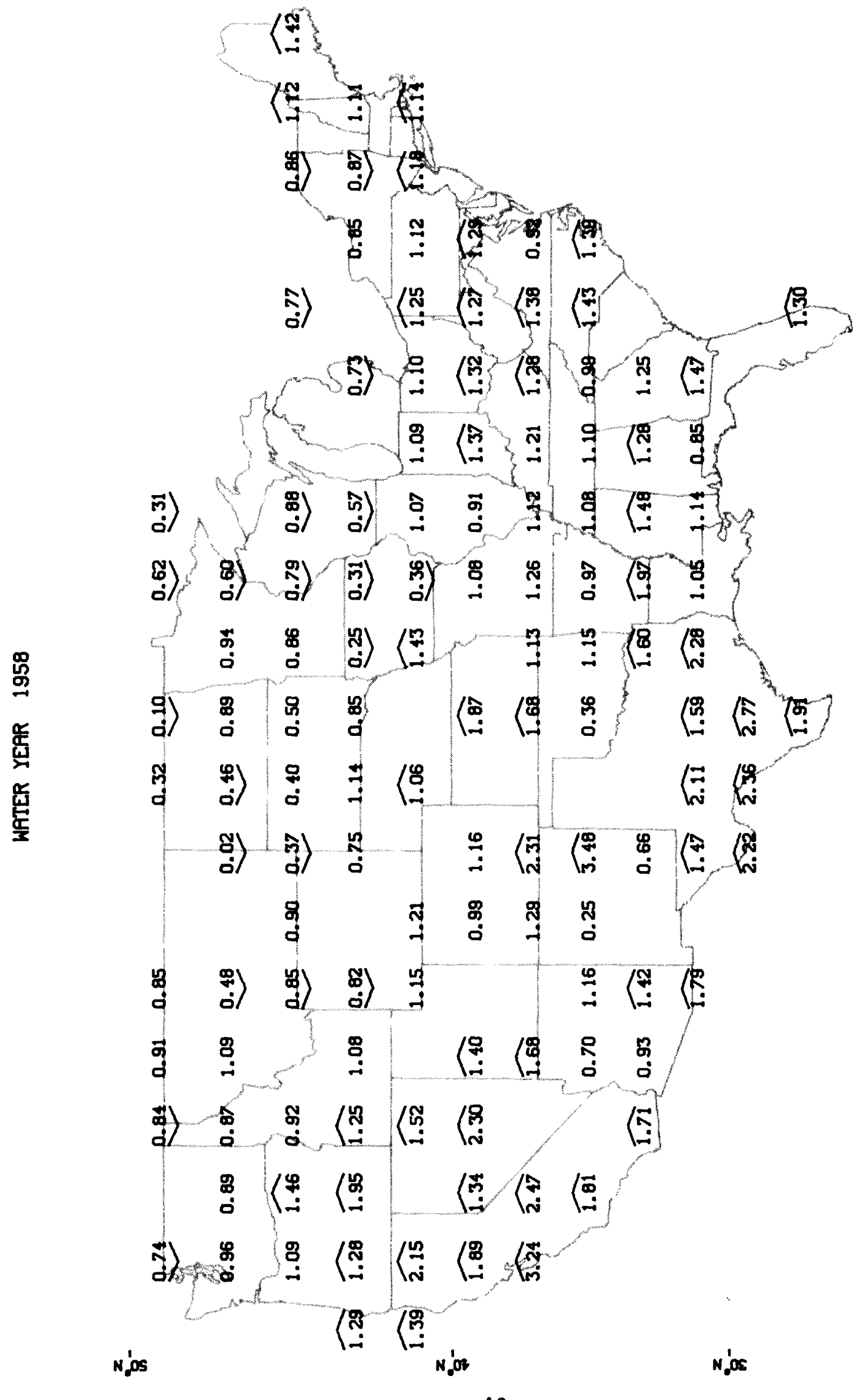

$-\frac{x}{2}$

$-\frac{7}{R}$

$-\frac{3}{8}$

$-\frac{x}{6}$

$-\frac{7}{8}$

$-\frac{7}{0}$

$-2$ 


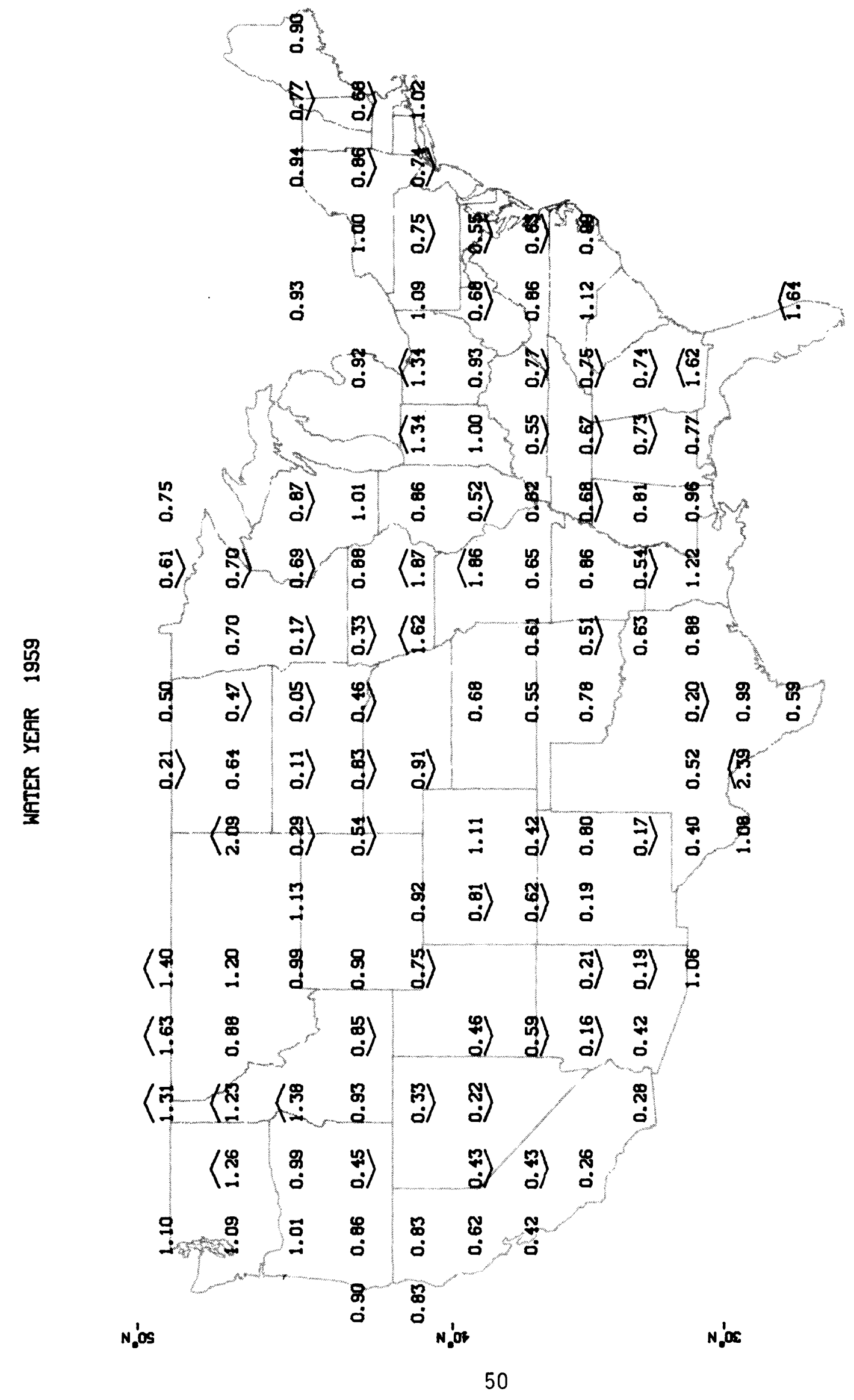

$-\frac{x}{2}$

$-\frac{2}{R}$

$-\frac{7}{8}$

$-\frac{7}{5}$

$-\frac{x}{8}$

$-\stackrel{7}{2}$

$-\underset{0}{2}$ 


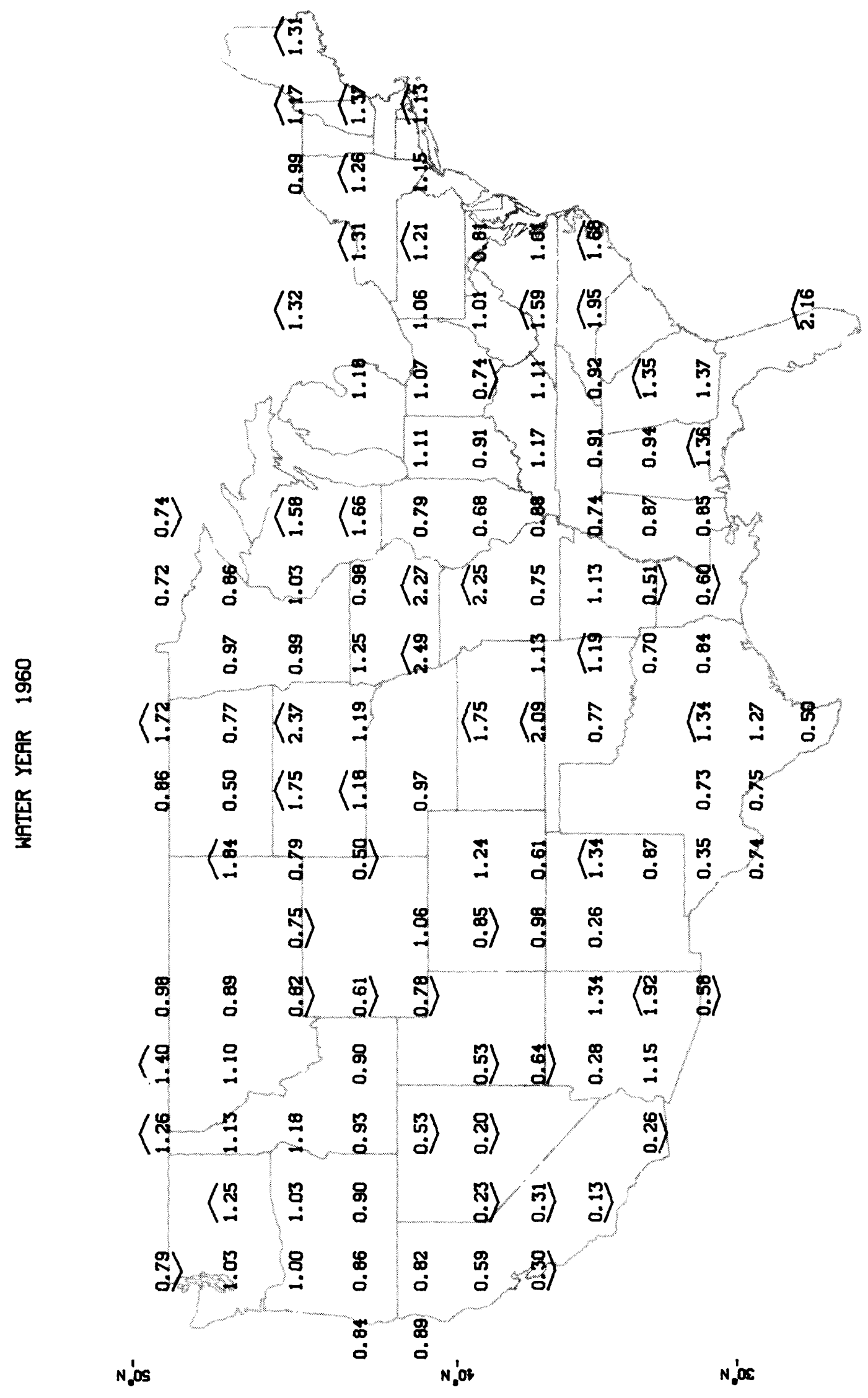




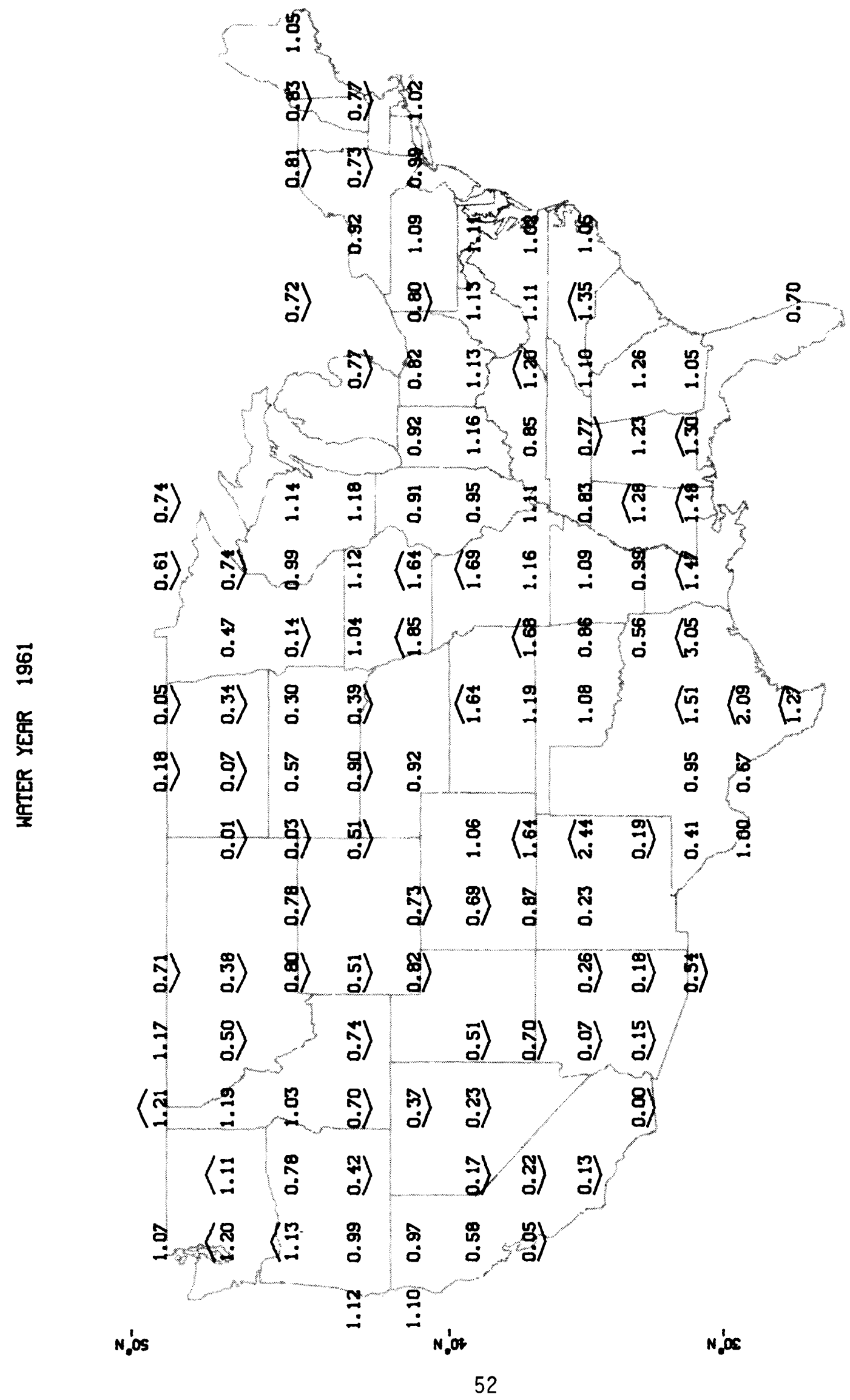




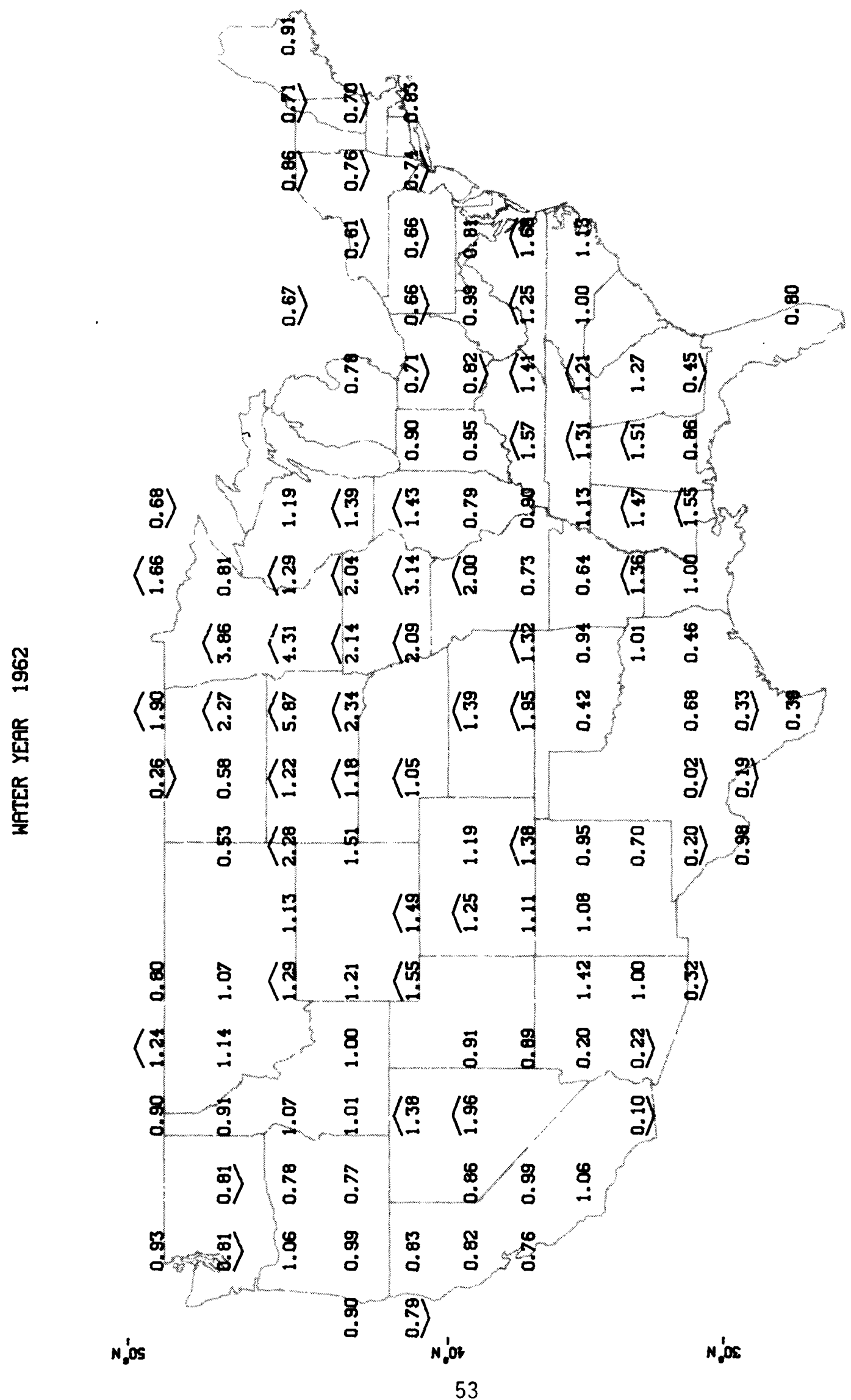




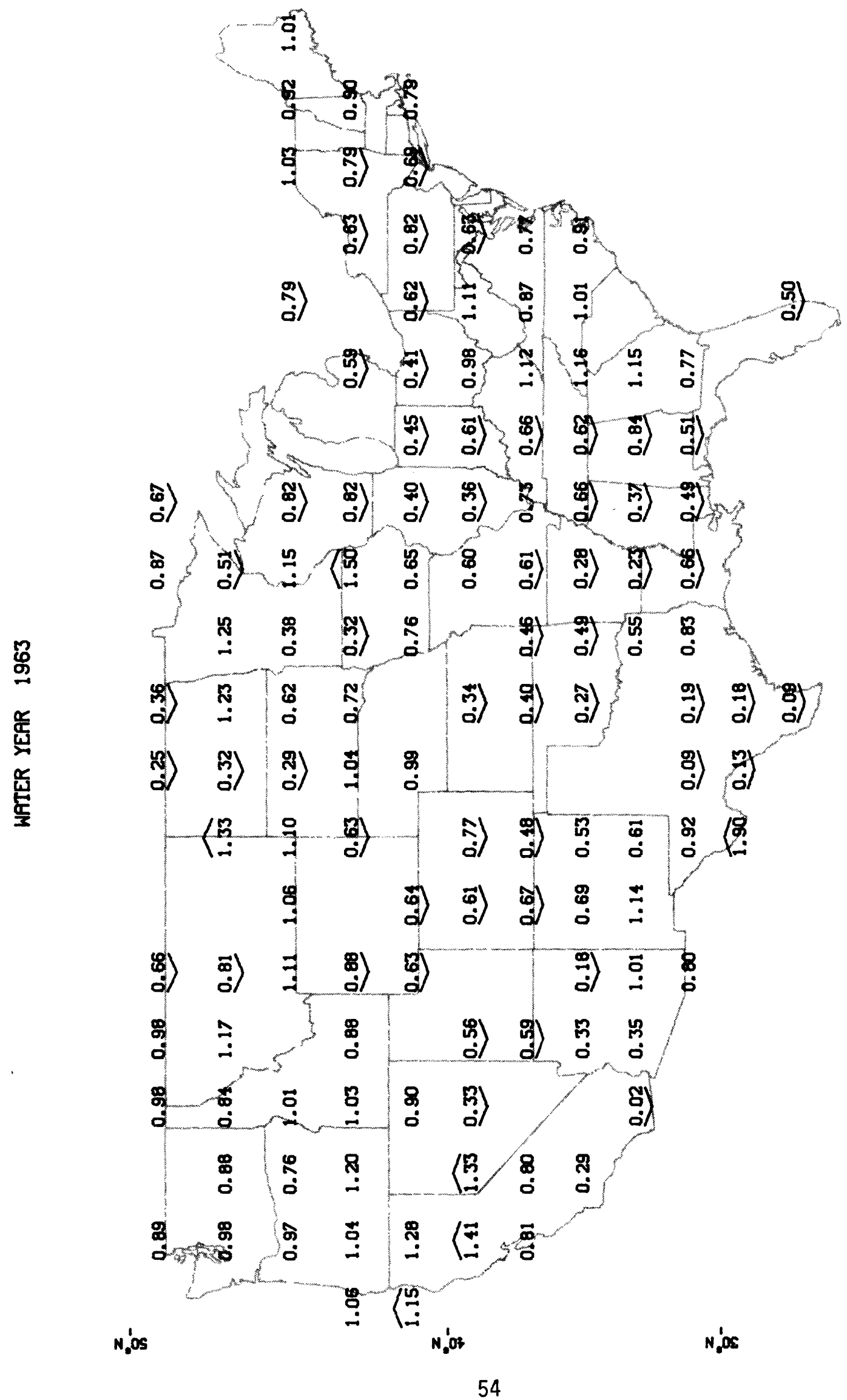




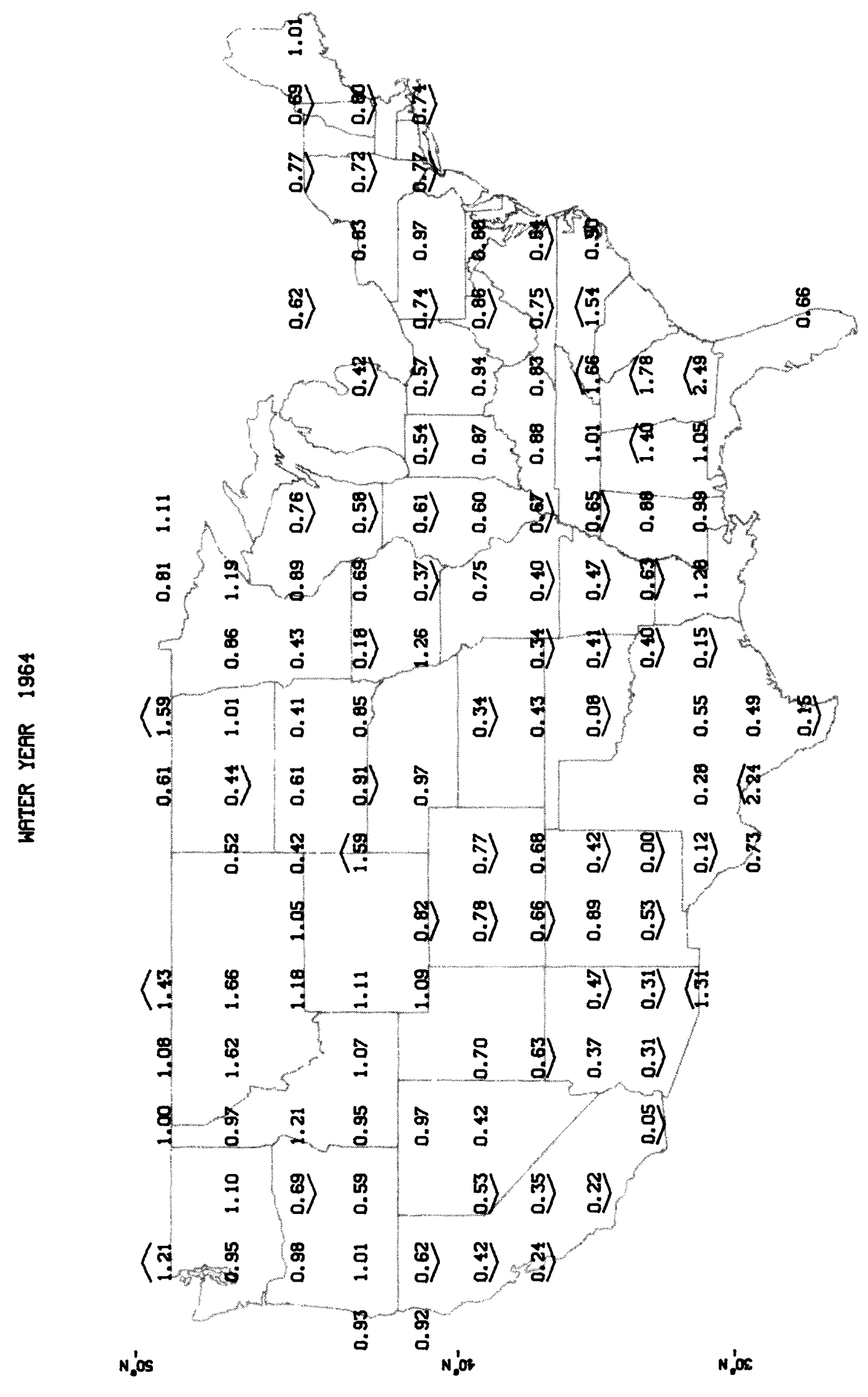

$-\frac{x}{2}$

$-\frac{x}{8}$

$-\frac{7}{8}$

$-\frac{1}{5}$

$-8$

$-2$

$-8$ 


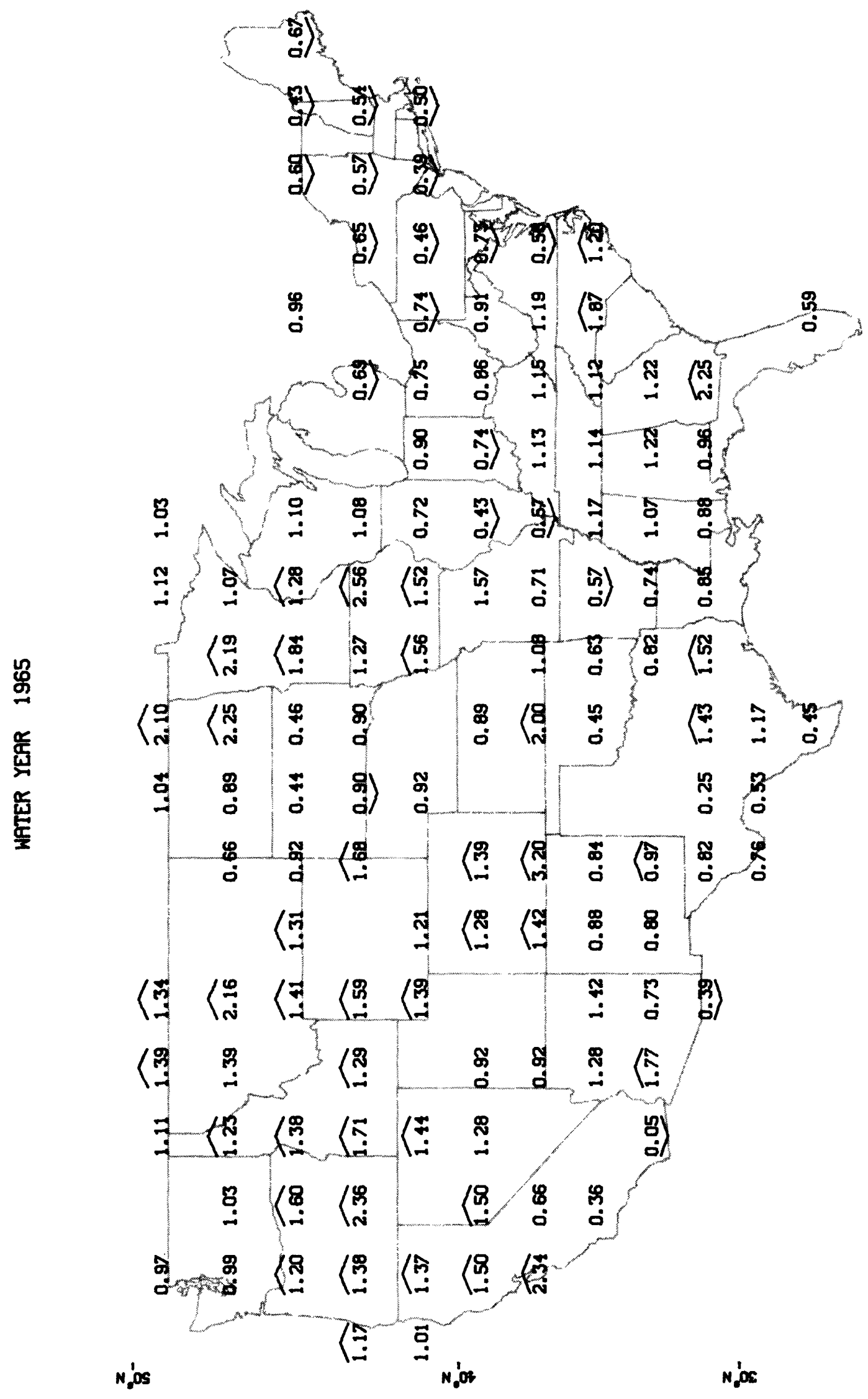

$-\frac{x}{2}$

$-7$

$-x^{2}$

$-\frac{2}{5}$

$-\frac{x}{5}$

-

$-\underset{z}{x}$ 


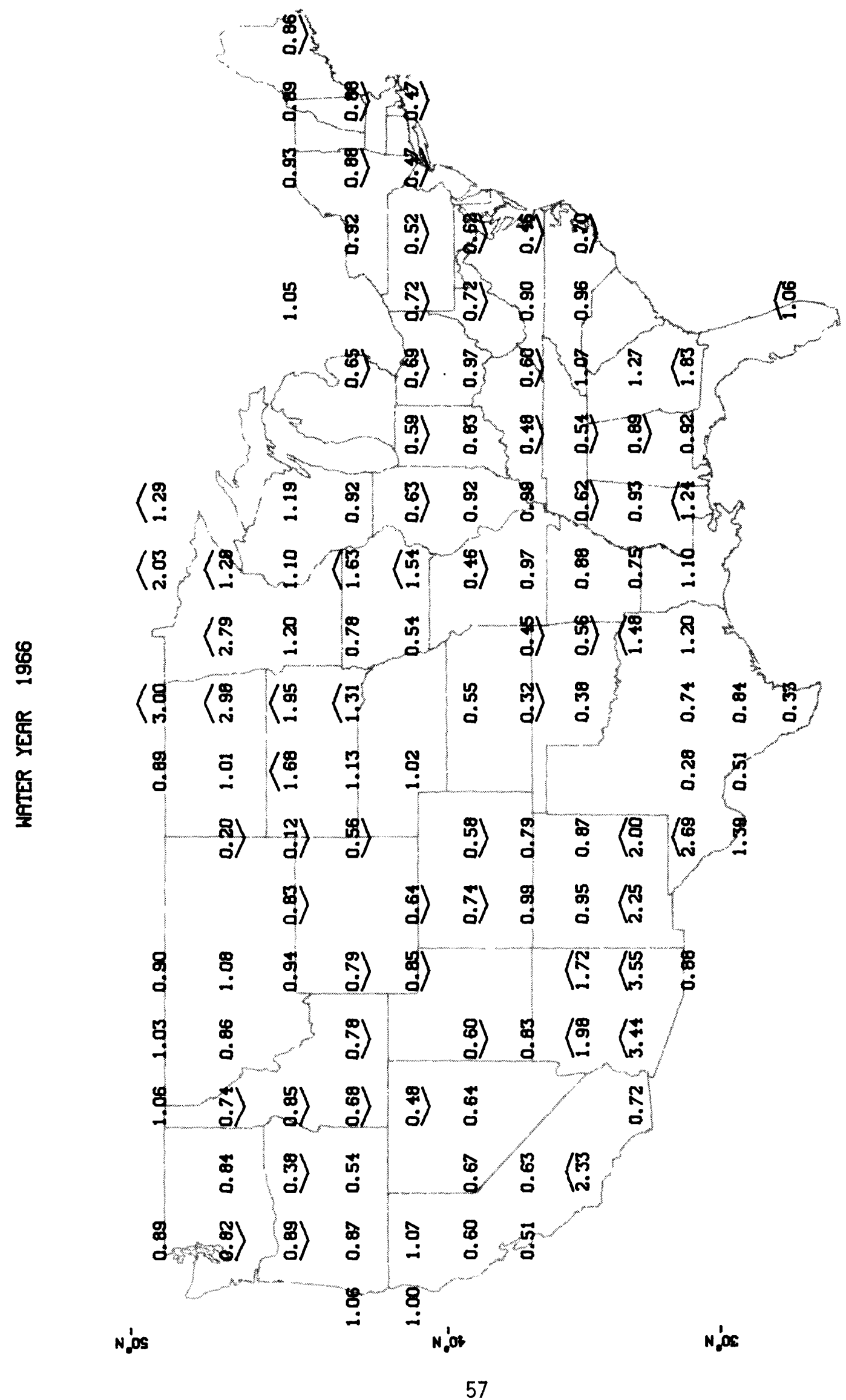




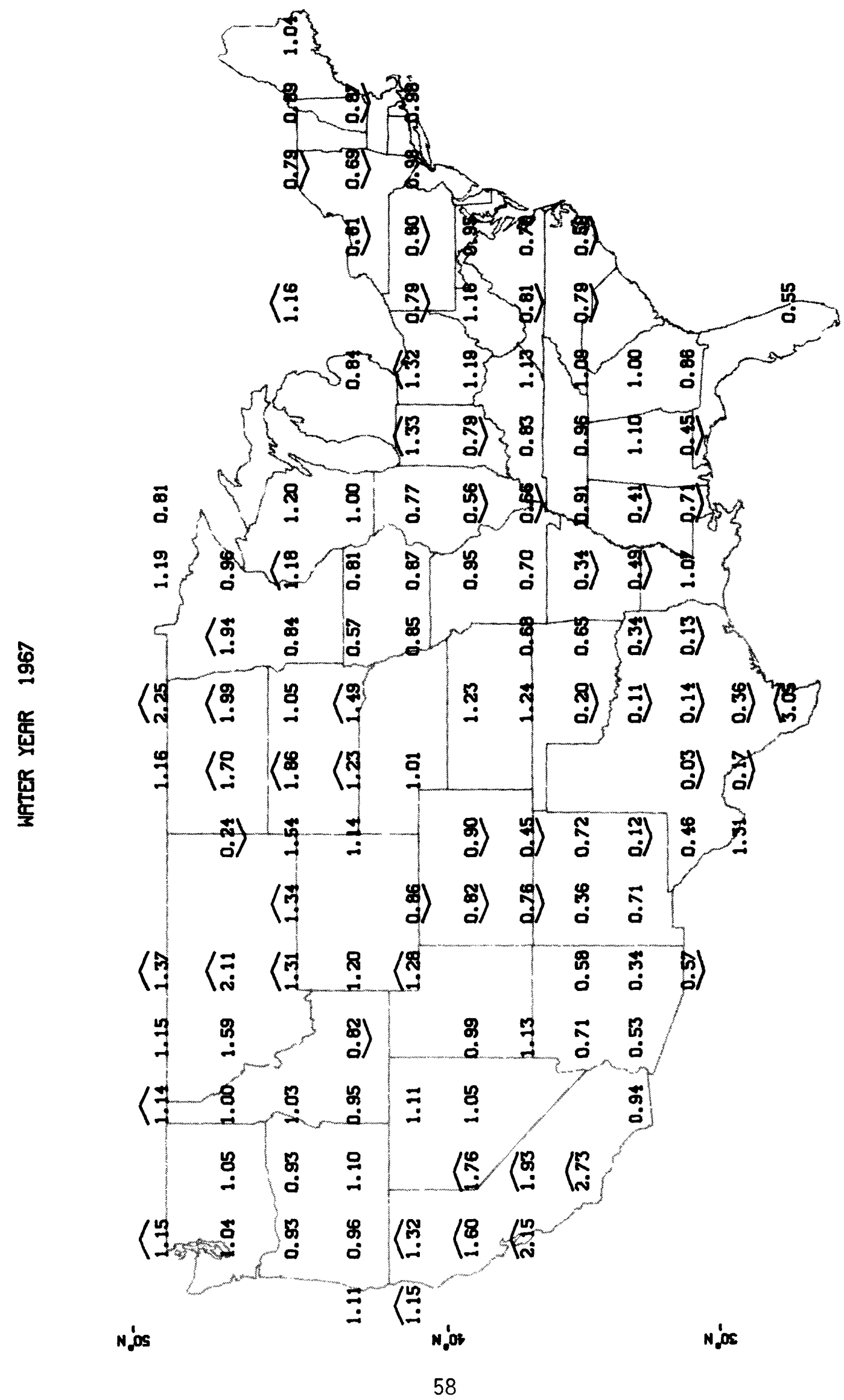

$-\frac{x}{R}$

$-\frac{x}{2}$

$-8$

$-2 x$

$-\frac{x}{8}$

$-2$

$x$
$-\infty$
0

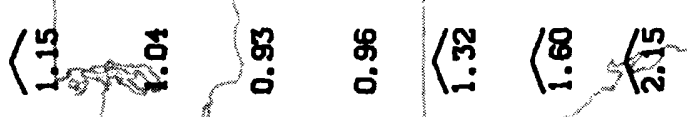

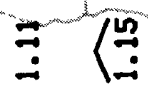




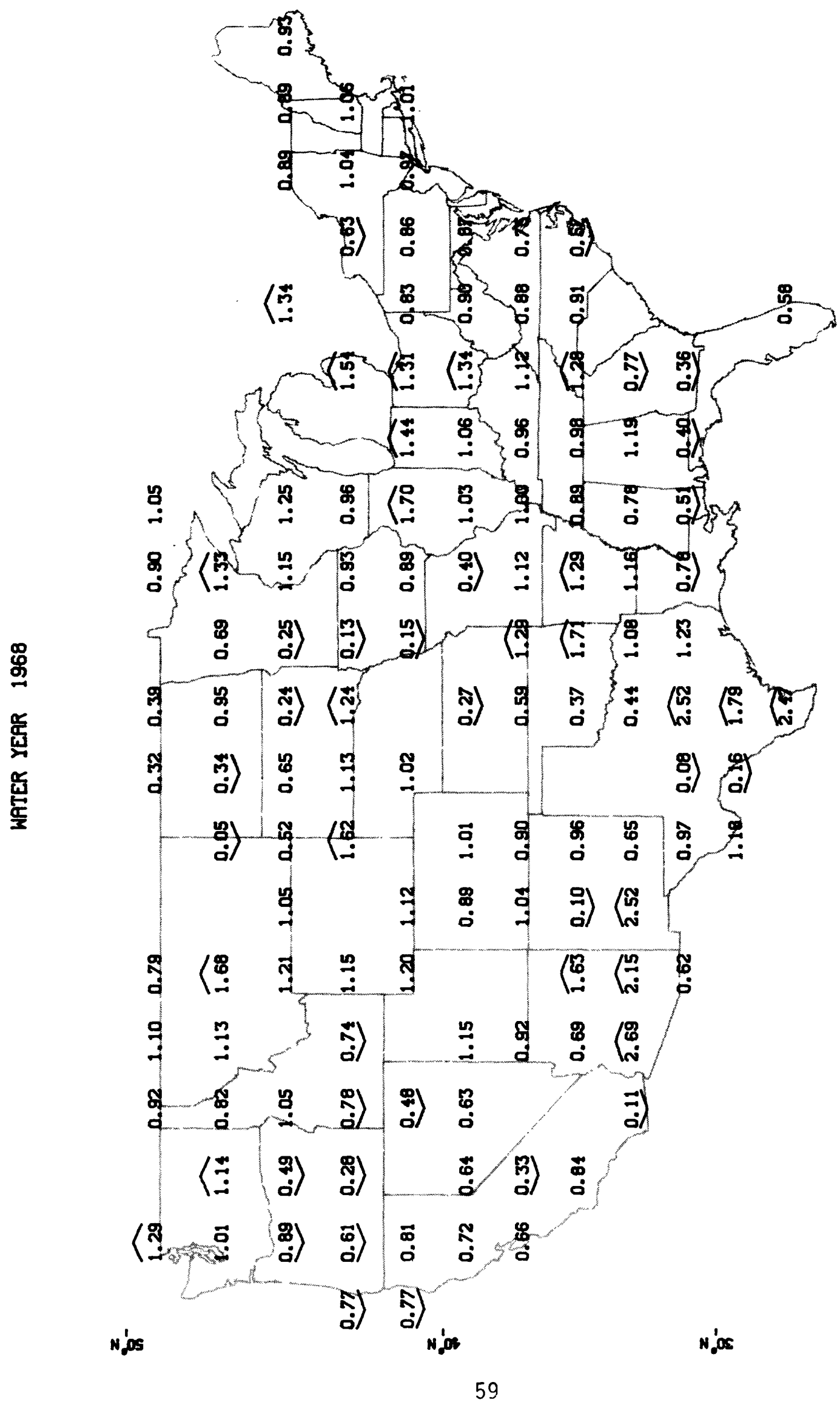

$-\frac{x}{2}$

$-\frac{2}{2}$

$-\frac{2}{8}$

$-\frac{7}{6}$

$-\frac{8}{8}$

$-\frac{2}{2}$

$-\underline{0}$ 


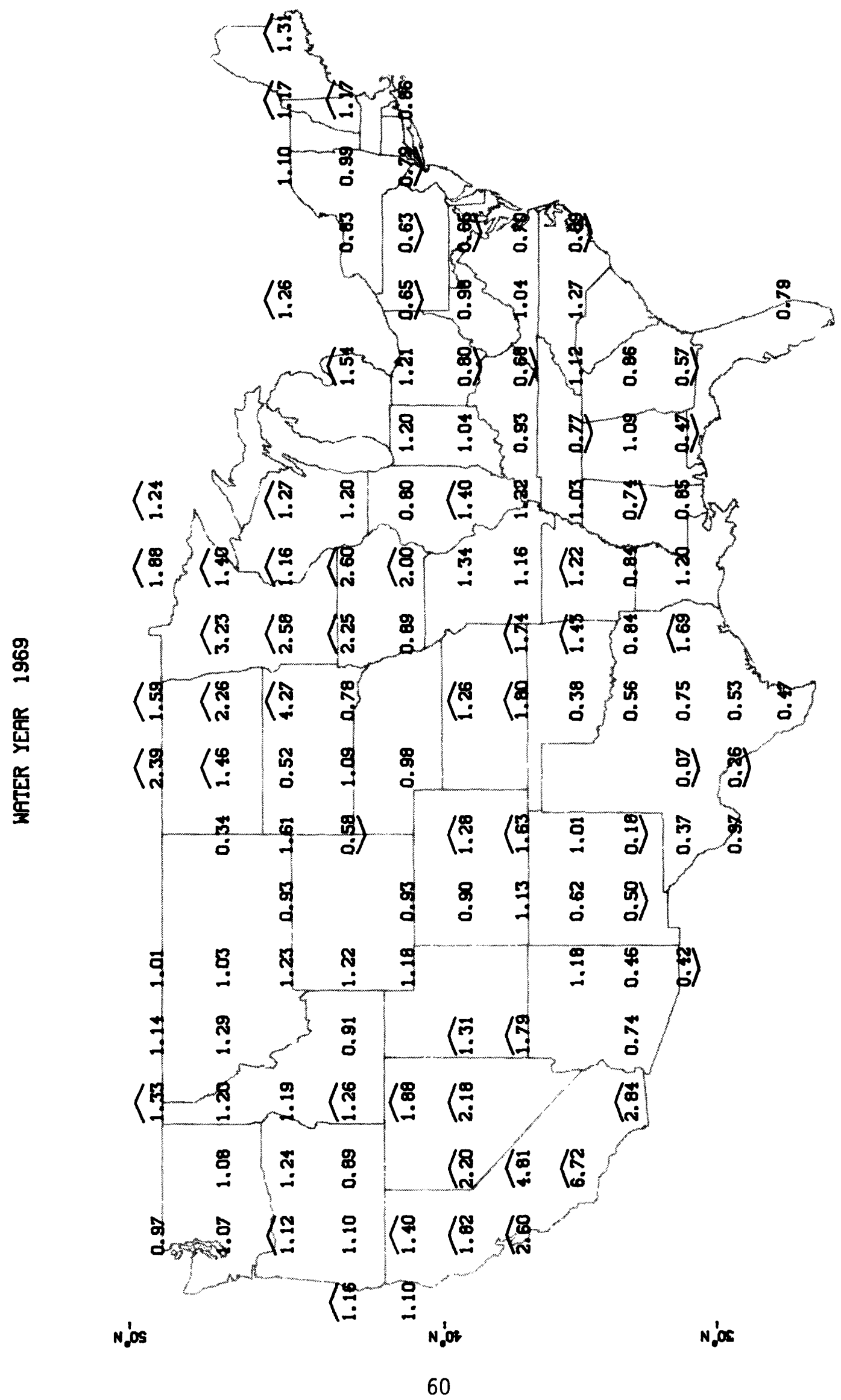

$-\frac{2}{2}$

$-\frac{x}{8}$

$-\frac{2}{8}$

$-\frac{2}{5}$

$-\frac{x}{8}$

$-\frac{x}{2}$

$-\stackrel{x}{=}$

$r^{28}$ 


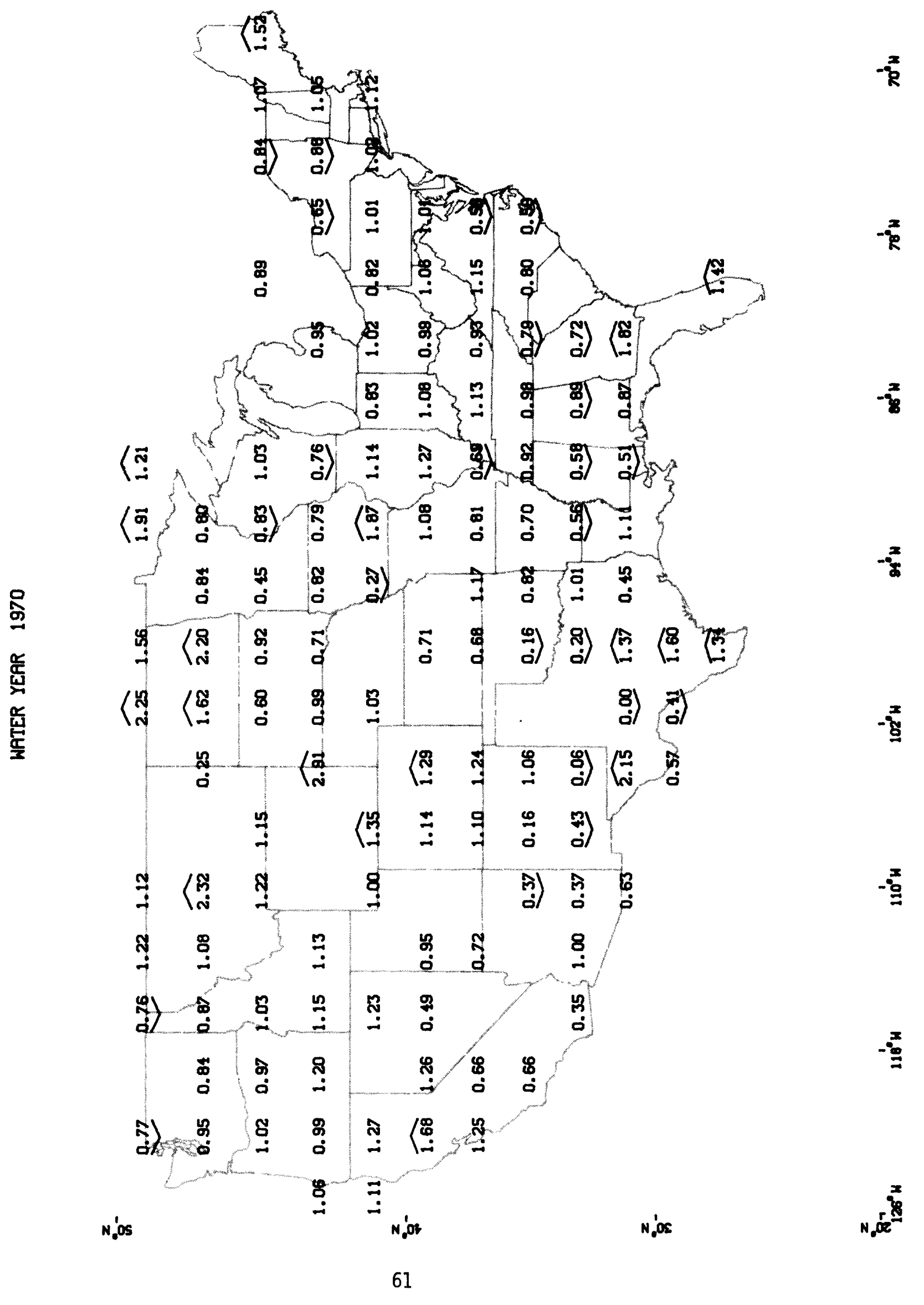




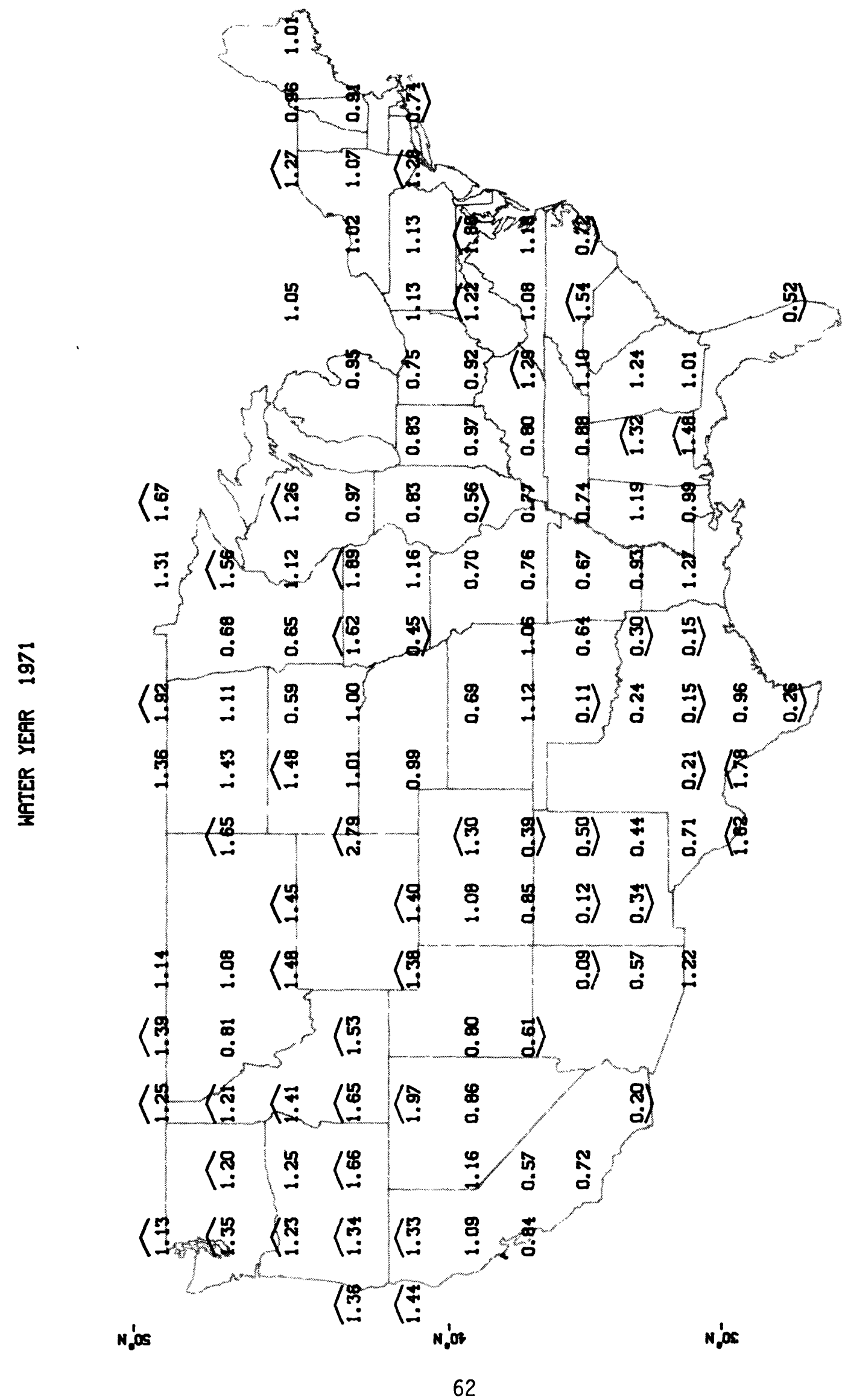




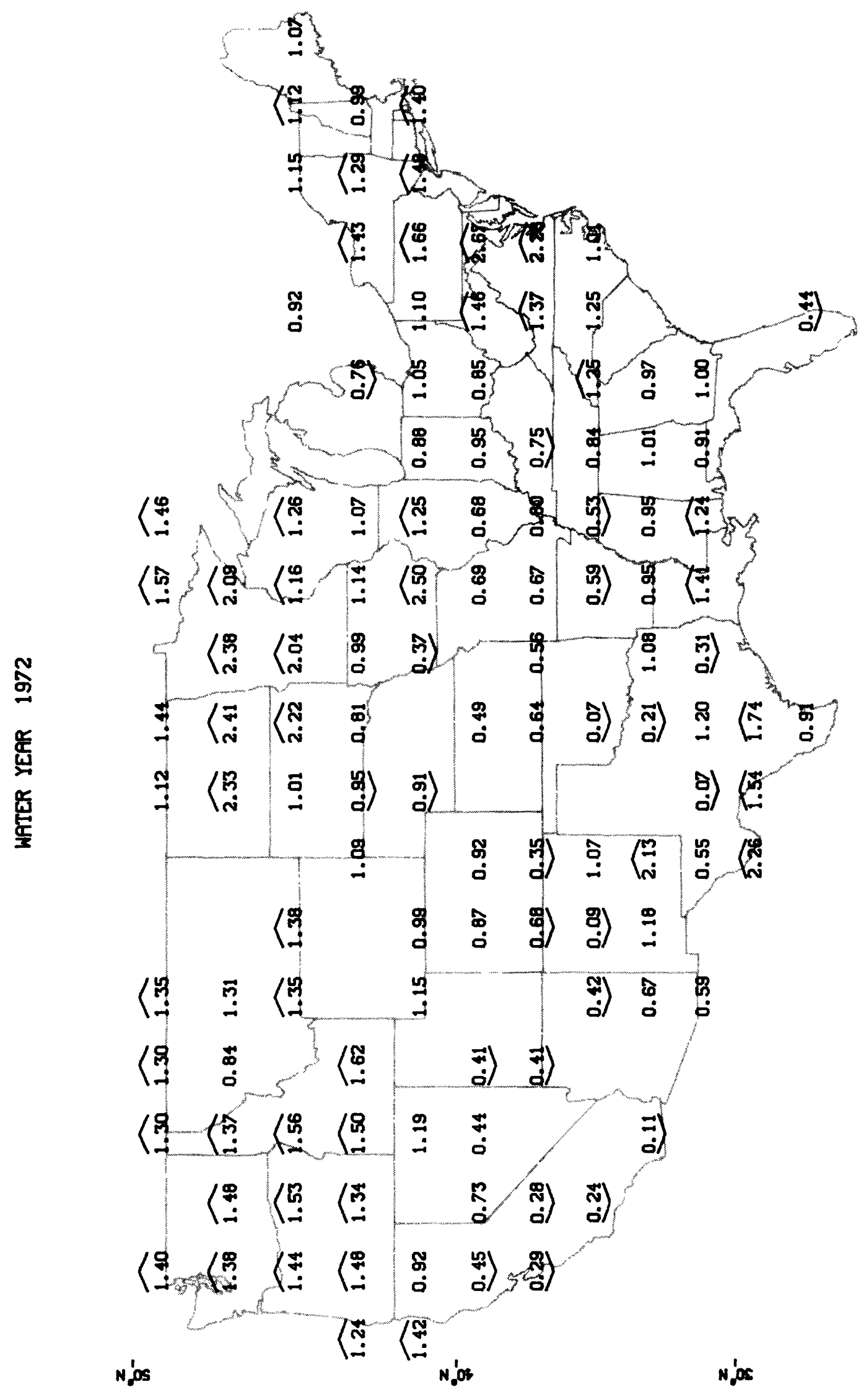

$-\frac{x}{R}$

$-\frac{x}{8}$

$-\frac{x}{8}$

$-\frac{x}{5}$

$\frac{x}{8}$

$-\frac{7}{9}$

- 


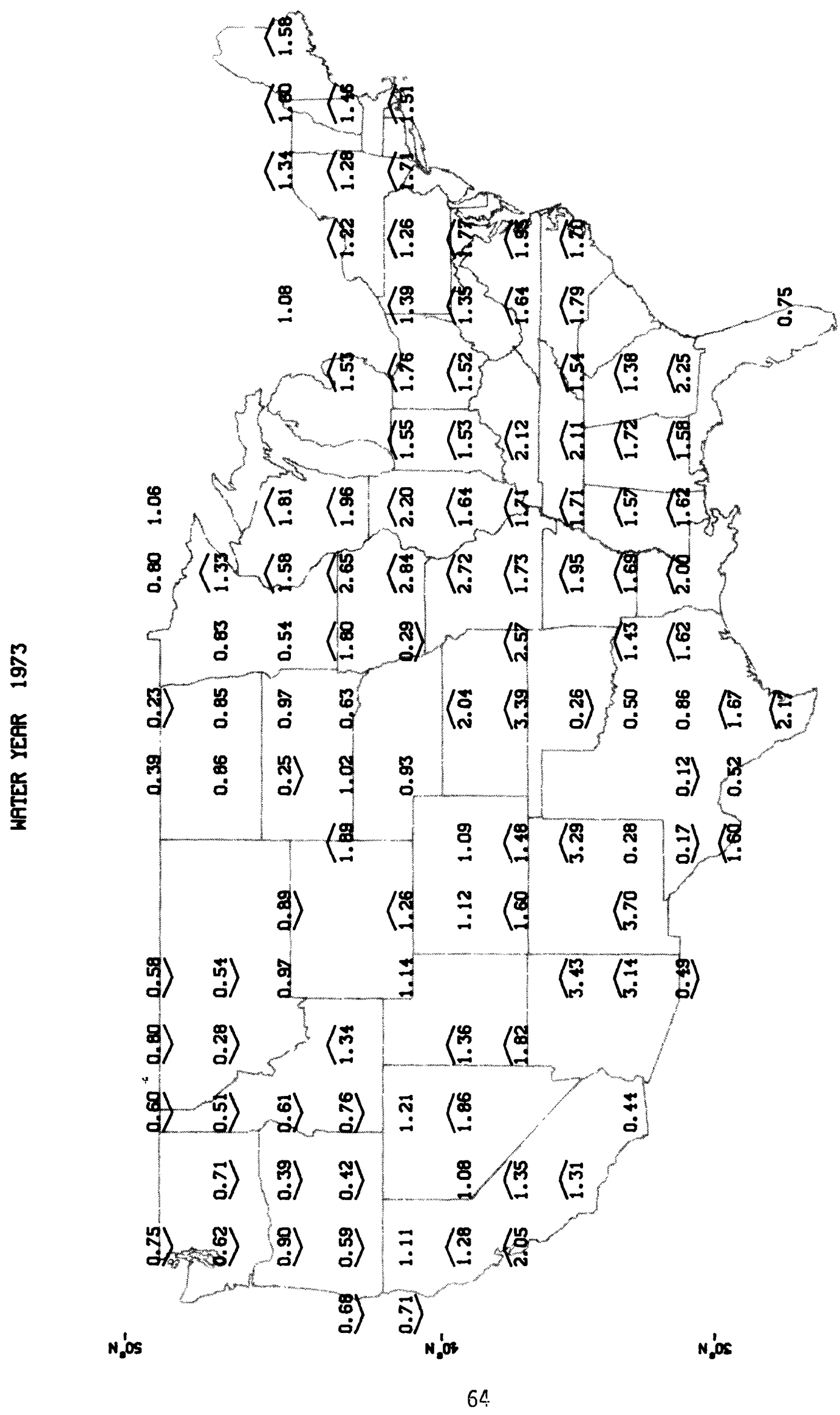

$-\frac{7}{R}$

$-\frac{x}{8}$

$-\frac{7}{8}$

$-\frac{2}{8}$

$-\frac{7}{8}$

$-\stackrel{7}{2}$

$-2$

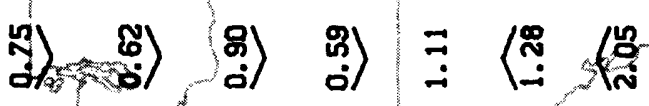

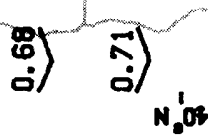




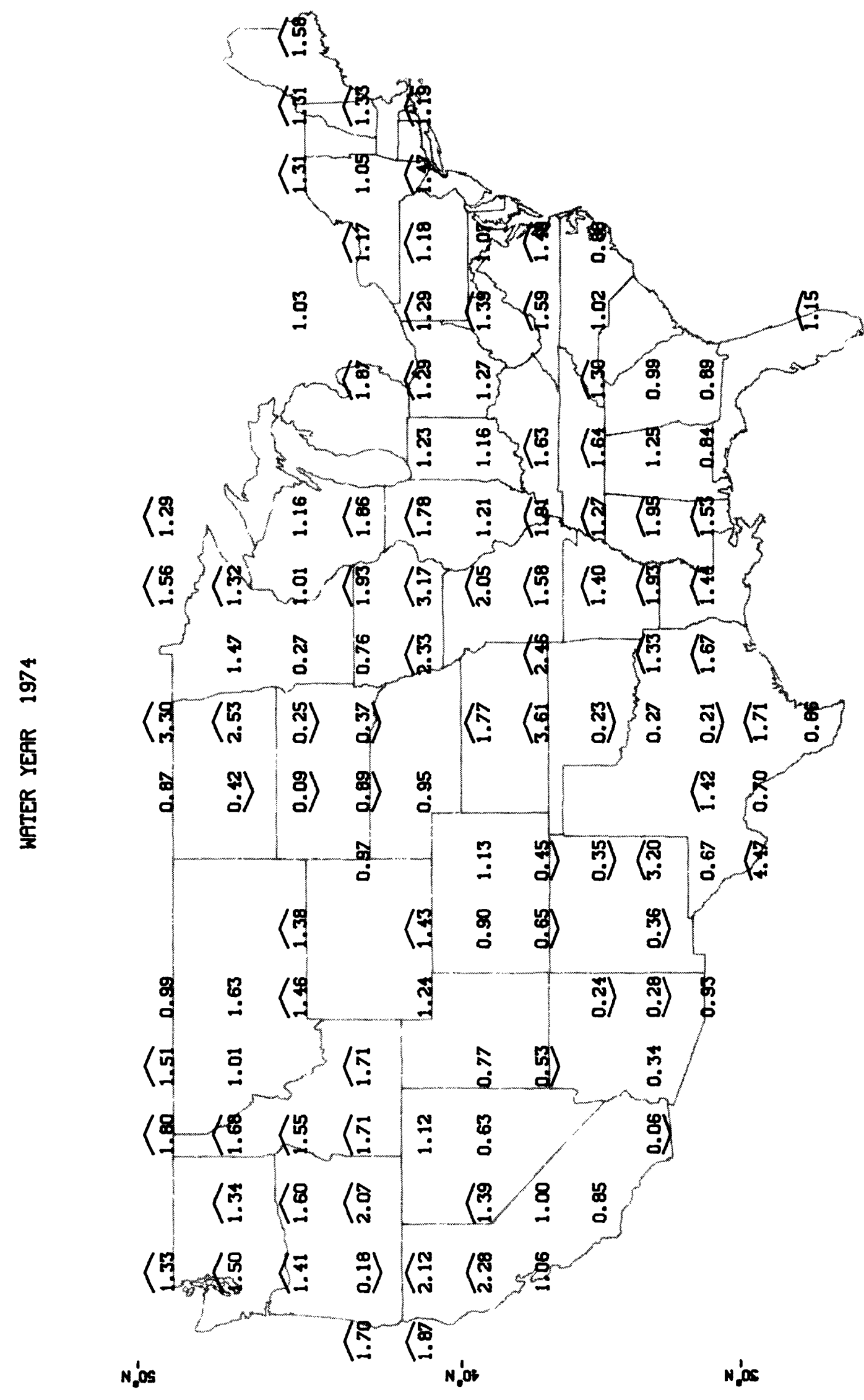

$-\frac{x}{R}$

$-\frac{x}{2}$

$-\frac{7}{8}$

$-2$

$-\frac{x}{8}$

$-\frac{x}{9}$

$-\frac{9}{9}$ 


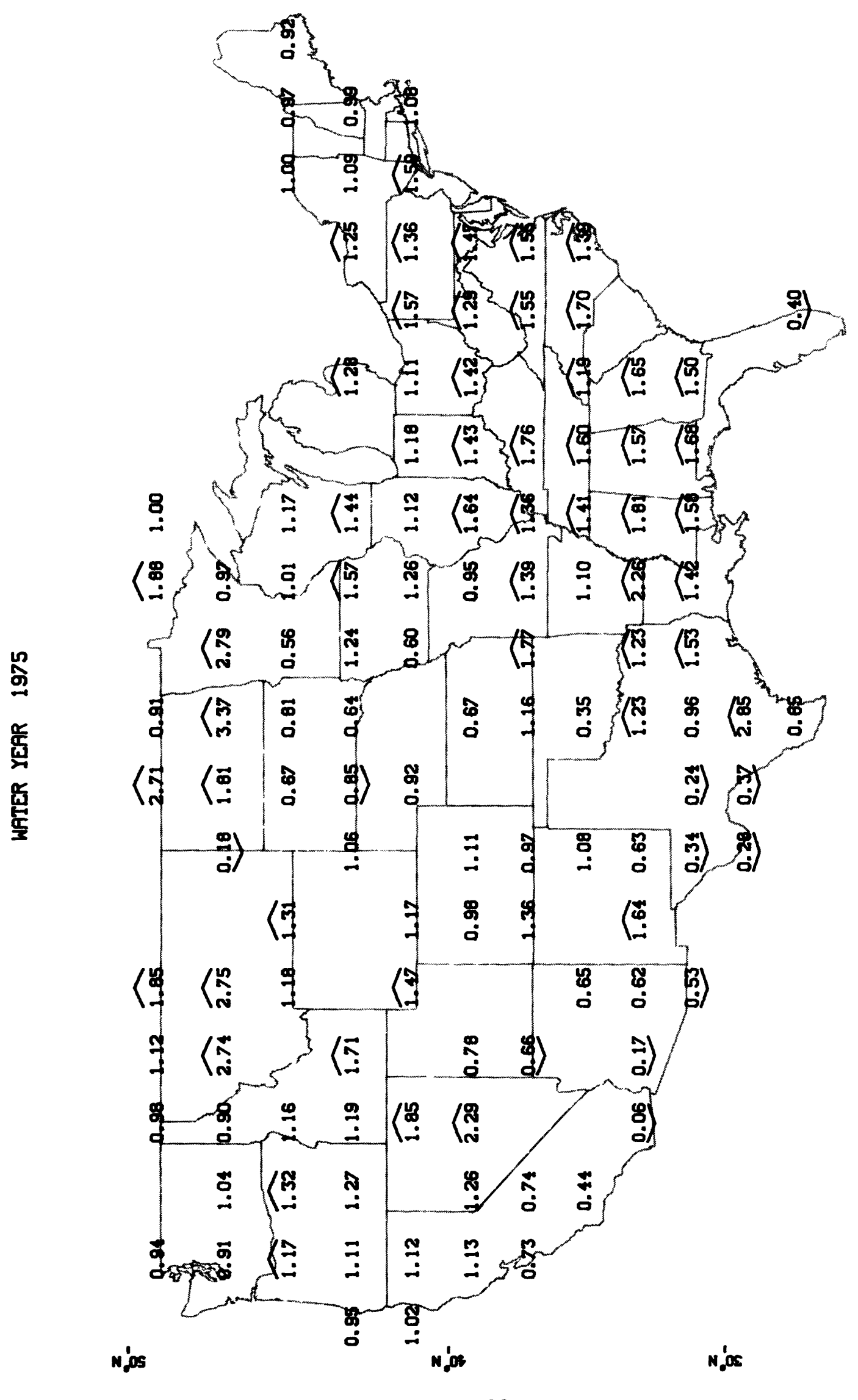

$-\bar{R}$

蛋

$-\frac{2}{8}$

$-\frac{x}{5}$

$-8$

$-\frac{2}{2}$

$-\stackrel{x}{=}$ 


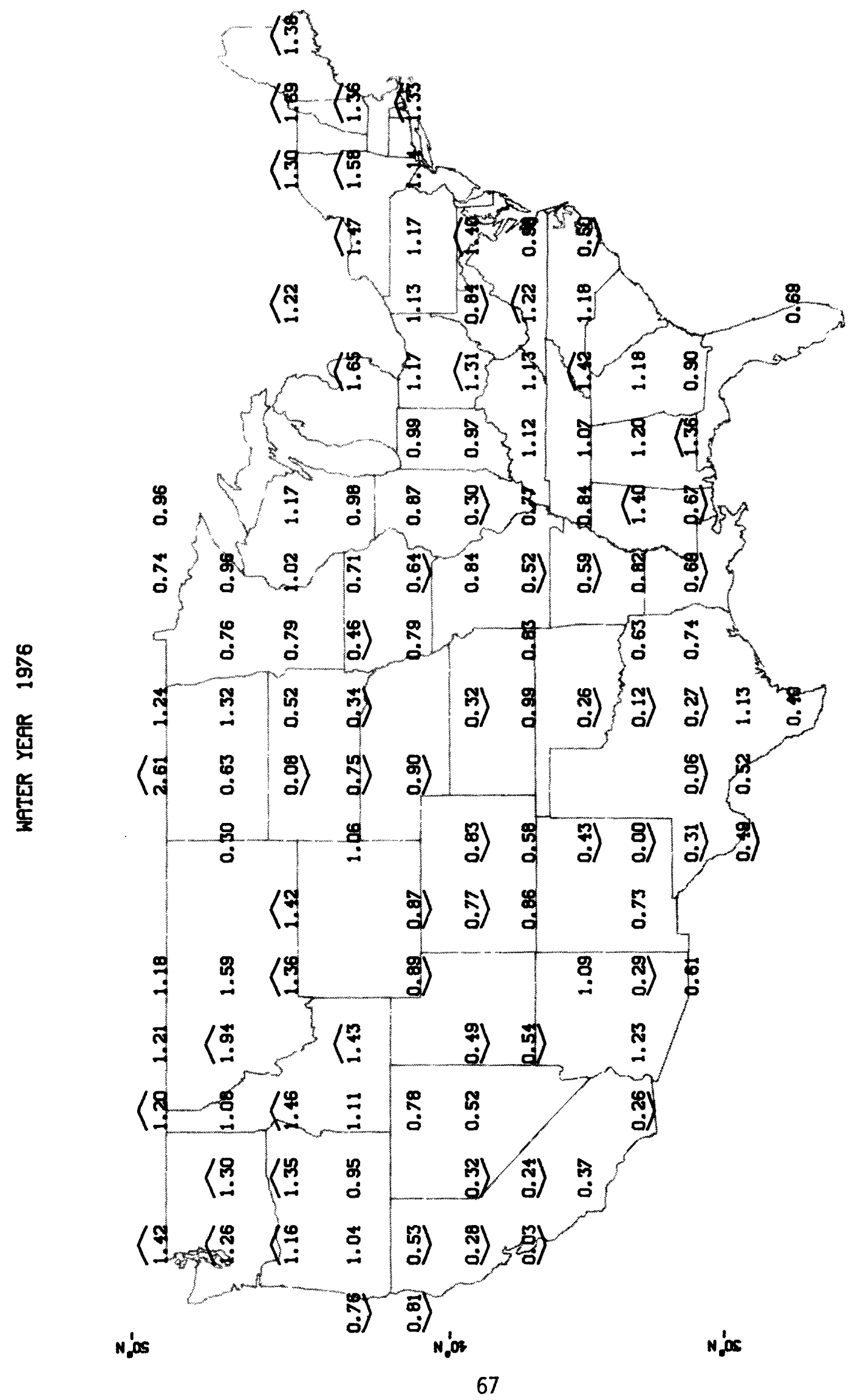

$-\frac{x}{R}$

$-\frac{x}{x}$

$-\frac{1}{8}$

$-\frac{x}{5}$

$-\frac{7}{8}$

$-\stackrel{2}{0}$

$-\frac{\mathbf{x}}{2}$

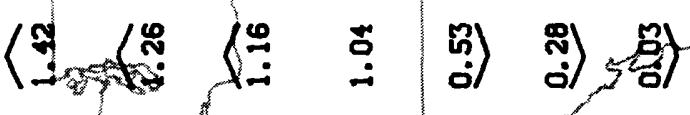




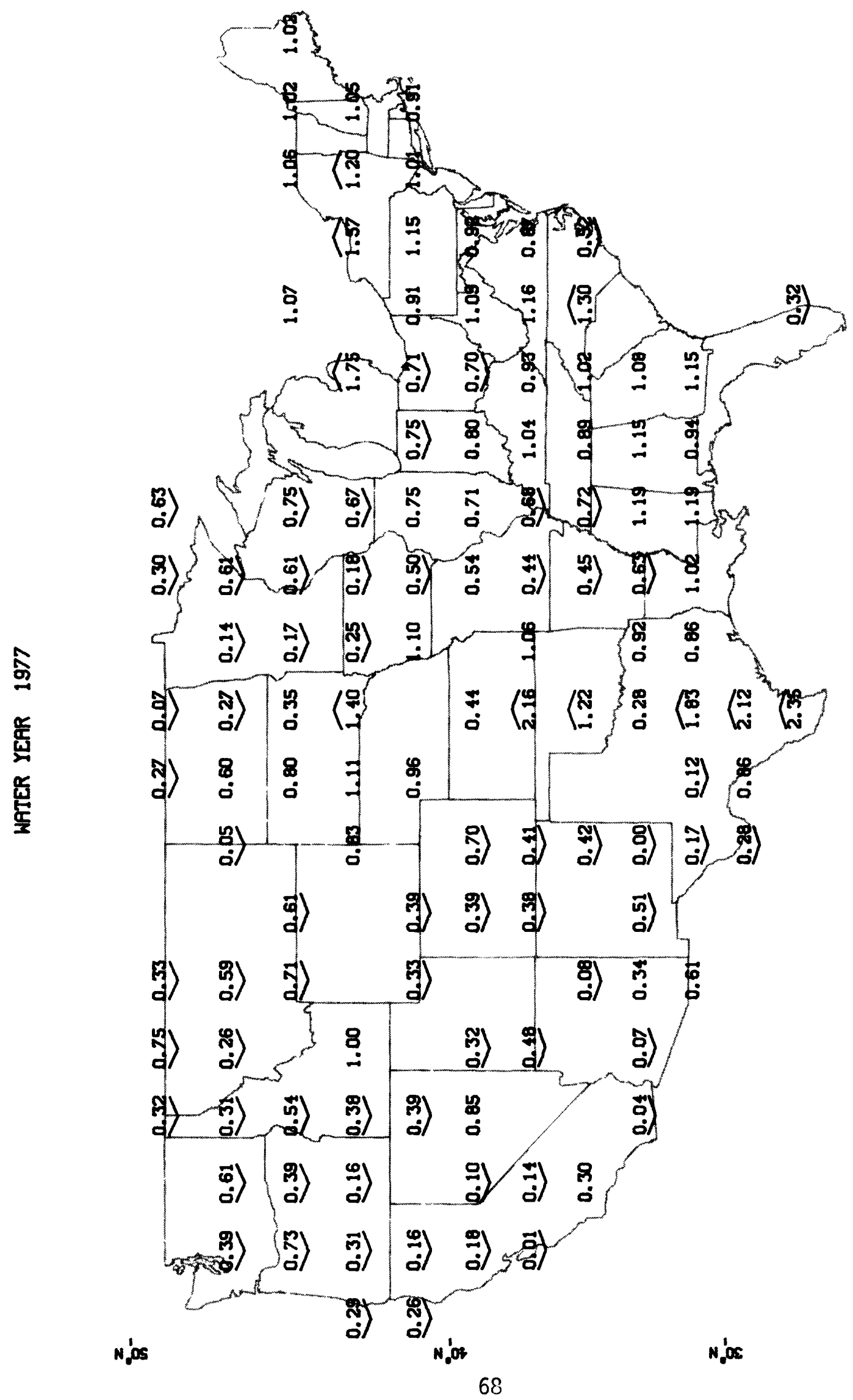

$-\frac{2}{\alpha}$

$-\frac{8}{x}$

$-8$

$-2$

- ชู

$-\frac{x}{2}$

$-\frac{x}{=}$

${ }^{x} e^{x}$ 


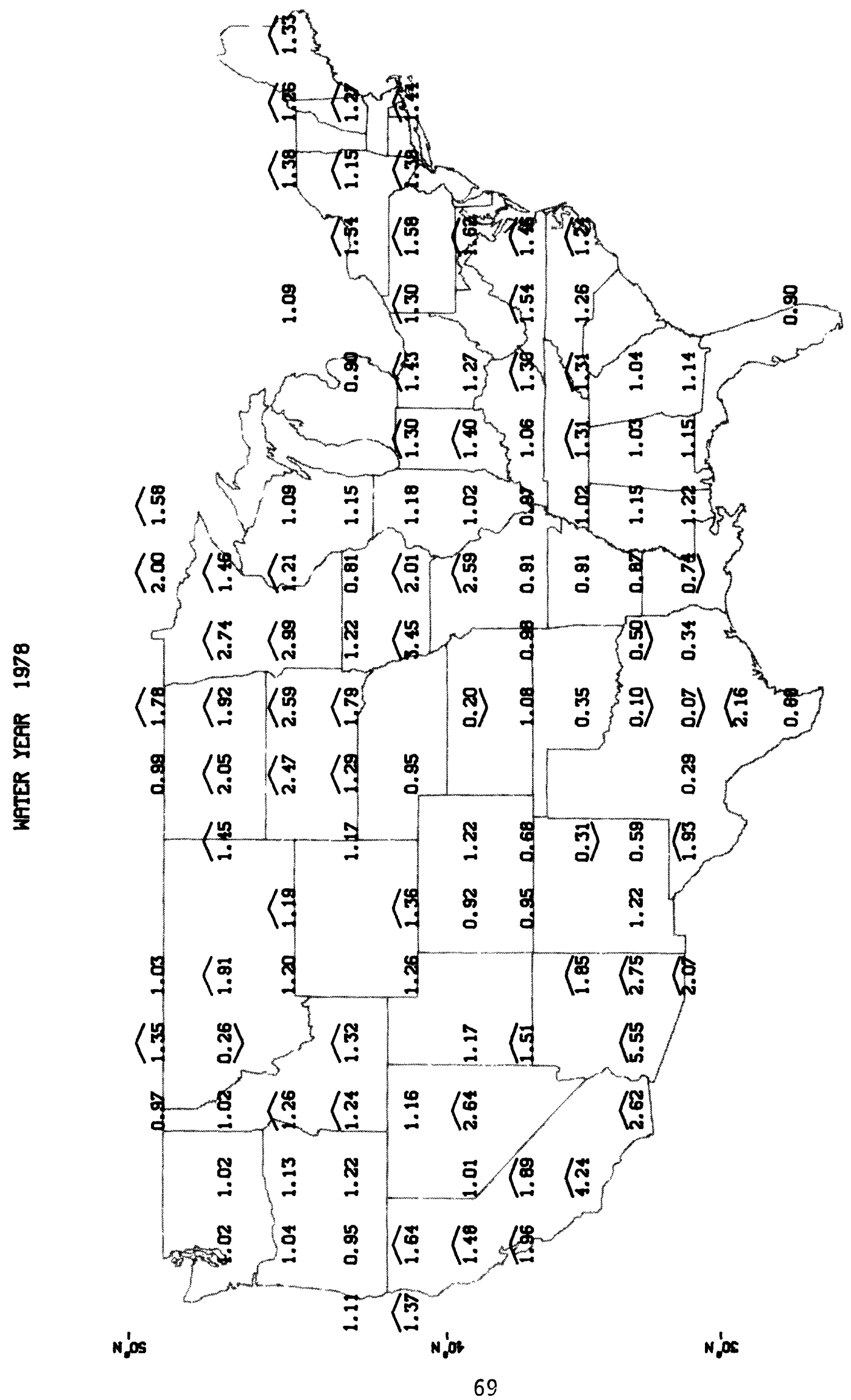

$-\frac{x}{2}$

$-\frac{x}{R}$

$-\frac{x}{8}$

$-\frac{7}{2}$

$-\frac{x}{8}$

$-8$

$-8$ 


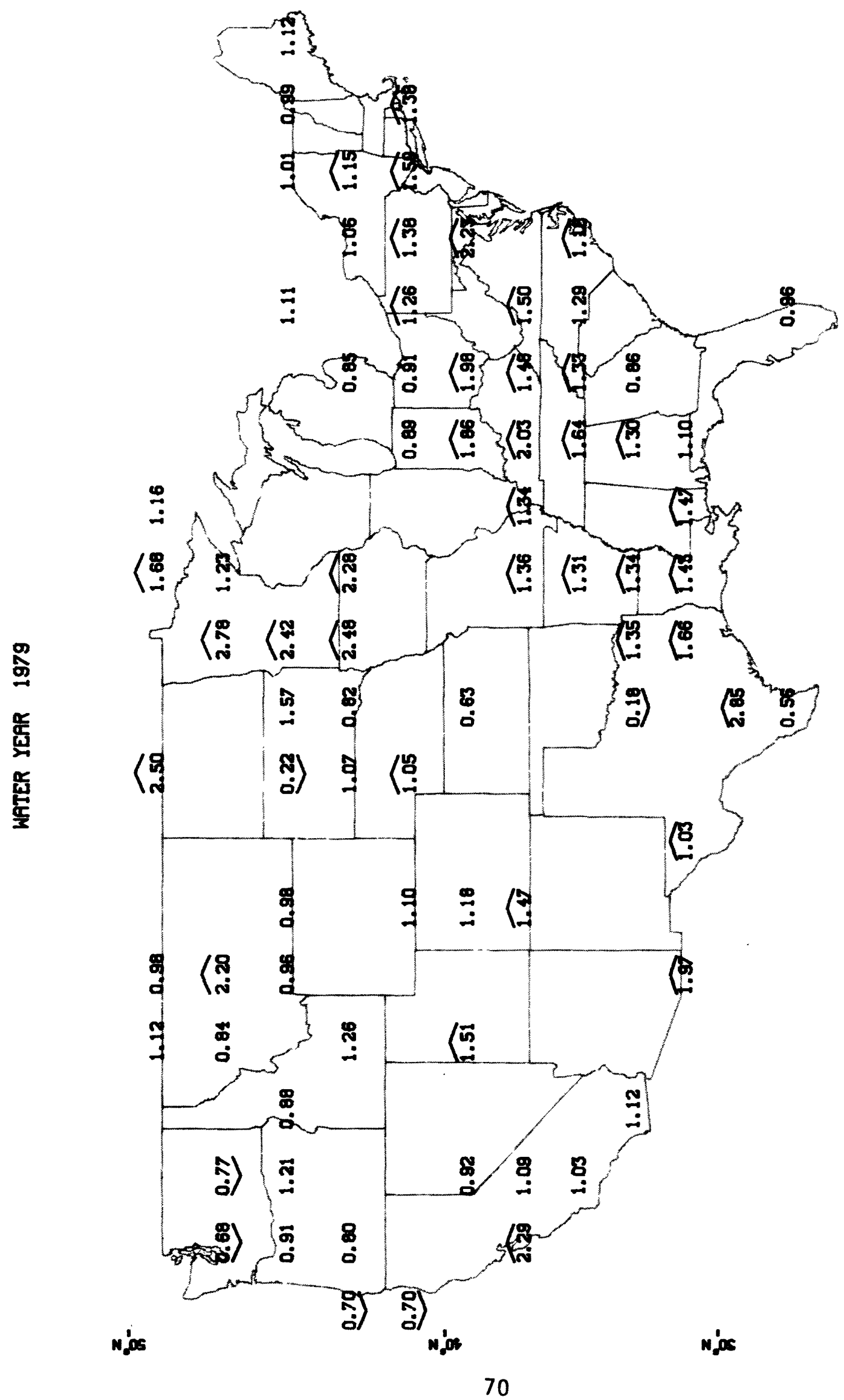

$-\frac{\pi}{k}$

$-2$

$-\frac{7}{8}$

$-\frac{2}{6}$

$-\frac{x}{5}$

$-\frac{x}{3}$

$-2$ 
Table 1.--Gaging Station Records Used

\begin{tabular}{|c|c|c|c|c|c|c|}
\hline Cell & Number & River & $\begin{array}{l}\text { Dr.Area } \\
\left(\mathrm{mi}^{2}\right)\end{array}$ & Class & $\begin{array}{l}\text { Normal } \\
\left(\mathrm{ft}^{3 / \mathrm{s}}\right)\end{array}$ & $\begin{array}{l}\text { Record used } \\
\text { (water years) }\end{array}$ \\
\hline $2 \mathrm{E}$ & 02256500 & Fisheating $\mathrm{Cr}$. & 311 & I & 281 & 1932- \\
\hline $2 \mathrm{E}$ & 02296750 & Peace $\mathrm{R}$ & 1367 & I & 1326 & $1932-$ \\
\hline $2 \mathrm{~K}$ & 08208000 & Atascosa $R$ & 1171 & II & 140 & $1925,1933-$ \\
\hline $3 \mathrm{~K}$ & 08167500 & Guadalupe $R$ & 1315 & II & 285 & $1923-$ \\
\hline 3L & 08449500 & Devils R & 4185 & I & 580 & $1911-14,1925-56$ \\
\hline 3L & 08455000 & Pinto $\mathrm{Cr}$. & 236 & I & 27.6 & $1957-$ \\
\hline 3L & 08449000 & Devils $R$ & 2730 & II & 190 & $1964-$ \\
\hline $3 M$ & 08374000 & Alamito $\mathrm{Cr}$. & 1504 & II & 21.5 & 1933- \\
\hline $4 \mathrm{~F}$ & 02231000 & St. Marys $\mathrm{R}$ & 700 & $\mathbf{I}$ & 608 & $1927-$ \\
\hline $4 \mathrm{~F}$ & 02352500 & Flint $\mathrm{R}$ & 5310 & II & 5830 & $1911-1920$ \\
\hline $4 \mathrm{~F}$ & 02329000 & Ochlocknee $\mathrm{R}$ & 1140 & I & 928 & $1927-$ \\
\hline $4 G$ & 02372500 & Conecuh $\mathrm{R}$ & 1344 & II & 1900 & $1911-19,1930-37$ \\
\hline $4 G$ & 02371500 & Conecuh R & 492 & I & 684 & $1938-$ \\
\hline $4 \mathrm{H}$ & 02479000 & Pascagoula $\mathrm{R}$ & 6600 & $\mathrm{I}$ & 9450 & $1931-38$ \\
\hline $4 \mathrm{H}$ & 07375500 & Tangiphoa $\mathrm{R}$ & 646 & I & 1078 & $1939-$ \\
\hline $4 I$ & 08013500 & Calcasieu R & 753 & II & 1150 & $1923-1924$ \\
\hline $4 \mathrm{I}$ & 08010000 & Bayou des Cannes & 131 & II & 250 & 1939 \\
\hline $4 \mathrm{~J}$ & 08033500 & Neches $R$ & 3637 & II & 2290 & $1911-24,1966-$ \\
\hline $4 \mathrm{~J}$ & 08110000 & Yegua Cr. & 1008 & II & 283 & $1925-65$ \\
\hline $4 \mathrm{~K}$ & 08095000 & N. Bosque $R$ & 968 & II & 221 & $1924-$ \\
\hline $4 \mathrm{~L}$ & 08126500 & Colorado $\mathrm{R}$ & 16840 & II & 318 & $1911-30$ \\
\hline $4 \mathrm{~L}$ & 08128500 & Middle Concho $\mathrm{R}$ & 1280 & II & 42 & $1931-60$ \\
\hline $4 \mathrm{~L}$ & 08134000 & N. Concho $\mathrm{R}$ & 1249 & II & 46 & $1961-$ \\
\hline 4M & 08408500 & Delaware $\mathrm{R}$ & 689 & I & 15 & $1913,1938-$ \\
\hline 40 & 09471000 & San Pedro $R$ & 1219 & II & 57.5 & $1913-$ \\
\hline $5 F$ & 02223500 & Oconee $\mathrm{R}$ & 4400 & II & 4440 & $1911-$ \\
\hline $5 G$ & 02416000 & Tallapoosa $R$ & 2460 & $\mathrm{I}$ & 3500 & $1911-13$ \\
\hline $5 G$ & 02407000 & Coosa R & 8390 & I, II & 12950 & $1914-28$ \\
\hline $5 G$ & 02450000 & Mulberry Fk. & 368 & I & 618 & $1929-$ \\
\hline $5 \mathrm{H}$ & 02486000 & Pear1 R & 3100 & II & 3786 & $1911-12,1971-$ \\
\hline $5 \mathrm{H}$ & 02487500 & Strong $R$ & 429 & $\mathbf{I}$ & 550 & $1929-1970$ \\
\hline $5 \mathrm{I}$ & 07369500 & Tensas $R$ & 309 & II & 334 & $1937-$ \\
\hline $5 \mathrm{~J}$ & 08022500 & Sabine R & 4858 & II & 3660 & $1911-59$ \\
\hline $5 \mathrm{~J}$ & 08061700 & Duck Cr. & 31.6 & II & 32 & $1960-$ \\
\hline $5 \mathrm{~K}$ & 08055500 & Elm Fk. Trinity $R$ & 2534 & II & 839 & $1911-32$ \\
\hline $5 \mathrm{~K}$ & 07314500 & Little Wichita R & 481 & II & 83 & $1933-55,1967$ \\
\hline $5 M$ & 08388000 & Rio Ruidoso & 290 & II & 19 & $1931-55$ \\
\hline $5 M$ & 08394500 & Rio Felix & 932 & II & 15 & $1951-$ \\
\hline $5 \mathrm{~N}$ & 09431500 & Gila R & 2860 & II & 170 & $1913-$ \\
\hline 50 & 09486000 & Rillito Cr. & 918 & III & 14 & $1911-29$ \\
\hline 50 & 09468500 & San Carlos $R$ & 1027 & II & 46.5 & $1930-$ \\
\hline $5 P$ & 09513000 & Aqua Fria $R$ & 1459 & II & 55 & $1915-19,1934-$ \\
\hline 50 & 11051500 & Santa Ana $R$ & 202 & III & 69 & $1911-30,1942-47$ \\
\hline 50 & 10258500 & Palm Canyon $\mathrm{Cr}$ & 93.3 & $\mathrm{I}$ & 4.87 & $1931-41,1948-$ \\
\hline
\end{tabular}


Table 1.--Gaging Station Records Used (continued)

\begin{tabular}{|c|c|c|c|c|c|c|}
\hline \multirow{2}{*}{$\begin{array}{l}\mathrm{Ce} 11 \\
6 \mathrm{E}\end{array}$} & \multirow{2}{*}{$\begin{array}{c}\text { Number } \\
02121000\end{array}$} & \multirow{2}{*}{$\begin{array}{c}\text { River } \\
\text { Yadkin R }\end{array}$} & \multirow{2}{*}{$\begin{array}{c}\text { Dr.Area } \\
\left(\mathrm{mi}^{2}\right) \\
3470\end{array}$} & \multirow{2}{*}{$\begin{array}{c}\text { Class } \\
\text { I }\end{array}$} & \multicolumn{2}{|c|}{$\begin{array}{l}\text { Normal Record used } \\
\left(\mathrm{ft}^{3 / \mathrm{s}}\right) \text { (water years) }\end{array}$} \\
\hline & & & & & 4300 & $1911-27$ \\
\hline $6 \mathrm{E}$ & 02132000 & Lynches $\mathrm{R}$ & 1030 & I & 934 & $1930-$ \\
\hline $6 \mathrm{~F}$ & 03451500 & French Broad R & 945 & $\mathrm{I}$ & 2058 & $1911-36$ \\
\hline $6 \mathrm{~F}$ & 02392000 & Etowah $\mathrm{R}$ & 605 & $\mathrm{I}$ & 1160 & $1937-$ \\
\hline $6 \mathrm{G}$ & 02396000 & Etowah R & 1810 & $\mathrm{I}$ & 2900 & $1911-20$ \\
\hline $6 G$ & 03602000 & Duck R & 2048 & II & 3150 & $1920-27$ \\
\hline $6 \mathrm{G}$ & 03604500 & Buffalo R & 707 & $\mathrm{I}$ & 1140 & $1928-$ \\
\hline $6 \mathrm{H}$ & 07030500 & Wolf R & 503 & $\mathrm{I}$ & 676 & $1930-71$ \\
\hline $6 \mathrm{H}$ & 07031650 & Wolf $R$ & 699 & $\mathrm{I}$ & 950 & $1971-$ \\
\hline $6 \mathrm{I}$ & 07260500 & Petit Jean R & 764 & $\mathrm{I}$ & 874 & $1917-45$ \\
\hline $6 \mathrm{I}$ & 07056000 & Buffalo R & 829 & $\mathrm{I}$ & 1110 & $1946-$ \\
\hline $6 \mathrm{~J}$ & 07336500 & Kiamichi R & 1423 & II & 1783 & $1926-71$ \\
\hline $6 \mathrm{~K}$ & 07331000 & Washita $\mathrm{R}$ & 7202 & II & 1540 & $1929-37$ \\
\hline $6 \mathrm{~K}$ & 07316500 & Washita R & 794 & II & 40 & $1938-$ \\
\hline $6 \mathrm{M}$ & 08380500 & Gallinas $\mathrm{Cr}$ & 84 & II & 16.6 & $1911-$ \\
\hline $6 \mathrm{~N}$ & 08342000 & Bluewater $\mathrm{Cr}$. & 235 & III & 9.7 & $1912-15,1916-18,1927$ \\
\hline 60 & 09509500 & Verde $\mathrm{R}$ & 6180 & II & 525 & $1911-29$ \\
\hline 60 & 09399000 & Clear $\mathrm{Cr}$ & 607 & II & 79 & $1930-$ \\
\hline $6 P$ & 09426500 & Bil1 Williams R & 5140 & II & 110 & $1914-15,1929-46$ \\
\hline $6 P$ & 09426000 & Bi11 Williams R & 4730 & II & 129 & $1940-68$ \\
\hline $6 \mathrm{R}$ & 11098000 & Arroyo Seco & 16.4 & II & 8.6 & $1914-15,1917-$ \\
\hline $7 \mathrm{D}$ & 02030500 & Slate $\mathrm{R}$ & 226 & I & 224 & $1927-$ \\
\hline $7 \mathrm{E}$ & 02112000 & Yadkin $\mathrm{R}$ & 493 & III & 746 & $1921-$ \\
\hline $7 \mathrm{E}$ & 02550000 & Roanoke R & 388 & I & 383 & $1911-1920$ \\
\hline $7 \mathrm{~F}$ & 03477000 & So. Fk. Holston $\mathrm{R}$ & 813 & I & 1175 & $1911-1930$ \\
\hline $7 \mathrm{~F}$ & 03409500 & Clear Fk. & 272 & I & 460 & $1931-71,1976-$ \\
\hline $7 G$ & 03433500 & Horpeth R & 408 & I & 545 & $1921-$ \\
\hline $7 \mathrm{H}$ & 05597000 & Big Muddy $R$ & 794 & I & 710 & 1912 only \\
\hline $7 \mathrm{H}$ & 07067000 & Current $\mathrm{R}$ & 1667 & I & 1850 & $1913-$ \\
\hline $7 \mathrm{I}$ & 06926000 & Osage $\mathrm{R}$ & 14000 & I & 9345 & $1911-25$ \\
\hline $7 \mathrm{I}$ & 06933500 & Gasconade $\mathrm{R}$ & 2840 & $\mathrm{I}$ & 2466 & $1924-$ \\
\hline $7 \mathrm{~J}$ & 07183000 & Neosho $R$ & 3818 & $\mathrm{I}$ & 1640 & $1918-24$ \\
\hline $7 \mathrm{~J}$ & 07187500 & Shoal Cr. & 439 & I & 380 & $1925-41$ \\
\hline $7 \mathrm{~J}$ & 07187000 & Shoal Cr. & 427 & I & 371 & $1942-$ \\
\hline $7 \mathrm{~K}$ & 07144200 & Little Arkansas $R$ & 1327 & III & 255 & $1923-$ \\
\hline $7 \mathrm{M}$ & 07208500 & Rayado $\mathrm{R}$ & 65 & I & 13 & Broken \\
\hline fN & 09361500 & Animas R & 692 & II & 757 & $1911-$ \\
\hline $7 \mathrm{~N}$ & 08246500 & Cone jos $\mathrm{R}$ & 282 & II & 312 & $1912-$ \\
\hline $7 \mathrm{P}$ & 09406000 & Virgin $\mathrm{R}$ & 934 & II & 178 & $1911-23$ \\
\hline $7 \mathrm{P}$ & 09403000 & Bright Angel $\mathrm{Cr}$. & 98.4 & II & 36.8 & $1924-73$ \\
\hline $7 \mathrm{P}$ & 09415000 & Virgin $\mathrm{R}$ & 5090 & III & 235 & $1974-$ \\
\hline $7 \mathrm{R}$ & 11222000 & ·Kings R & 1694 & II & 2240 & $1911-50$ \\
\hline $7 \mathrm{R}$ & 11213500 & Kings $\mathrm{R}$ & 952 & $\mathrm{I}$ & 1410 & $1927-28,1932-$ \\
\hline $7 \mathrm{R}$ & 11224500 & Los Gatos Cr. & 95.5 & II & 5.35 & $1946-$ \\
\hline 7R & 11210500 & Kaweah R & 520 & II & 571 & $1951-61$ \\
\hline $7 S$ & 11152000 & Arroyo Seco & 241 & I & 135 & $1911-40$ \\
\hline $7 \mathrm{~S}$ & 11312000 & Bear $\mathrm{Cr}$. & 47.6 & I & 11.5 & $1931-$ \\
\hline
\end{tabular}


Table 1.--Gaging Station Records Used (continued)

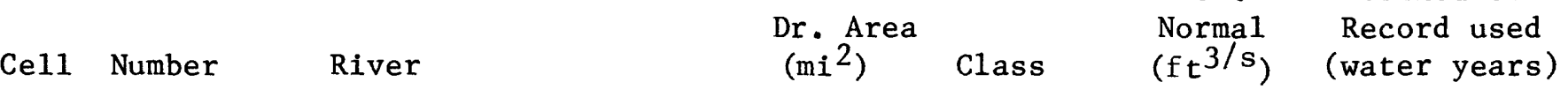

$\begin{array}{cc}\text { Ref. } & \text { Period of } \\ \text { Normal } & \text { Record used } \\ \left(\mathrm{ft} \mathrm{t}^{3 / \mathrm{s}}\right) & \text { (water years) }\end{array}$

$8 \mathrm{D}$
$8 \mathrm{D}$
$8 \mathrm{E}$
$8 \mathrm{E}$
$8 \mathrm{~F}$
$8 \mathrm{~F}$
$8 \mathrm{G}$
$8 \mathrm{G}$
$8 \mathrm{H}$
$8 \mathrm{H}$
$8 \mathrm{I}$
$8 \mathrm{~K}$
$8 \mathrm{M}$
$8 \mathrm{M}$
$8 \mathrm{~N}$
$8 \mathrm{P}$
$8 \mathrm{O}$
8

01668000

01645000

03051000

03186500

03159500

03157500

03373500

03303000

03380500

07016500

06897500

06864500

07101000

06725500

09085000

10234500

10325500

10312000

11335000

11384000

01127000

01439500

01396500

01548000

01573000

03021500

03109500

04193500

03326500

03328000

05572000

05454500

05455000

06800500

06813000

06687000

09239500

10168500

09266500

10153800

10322500

10329500

11525500

Rappahannock R
Seneca Cr.
Tygart Valley R
Williams R
Hocking R
Hocking R
E. Fk. White R
Blue R
Skillet Fk.
Bourbeuse R
Grand R
Smoky Hill R
Lion Cr.
Middle Boulder Cr.
Roaring Fk.
Beaver R
Reese R
Carson R
Cosumnes R
Big Chico Cr.

Quinebaug $\mathrm{R}$

Bush Kill

So $\mathrm{Br}$. Raritan $\mathrm{R}$

No. Bald Eagle Cr.

Swatara $\mathrm{Cr}$.

French $\mathrm{Cr}$.

Little Beaver $\mathrm{Cr}$.

Maumee $\mathrm{R}$

Mississinewa $\mathrm{R}$

Ee1 R

Sangaman $\mathrm{R}$

Iowa $\mathrm{R}$

Ralston $\mathrm{Cr}$.

Elkhorn R

Tarkio $R$

Blue $\mathrm{Cr}$.

Yampa R

Big Cottonwood $\mathrm{Cr}$.

Ashley $\mathrm{Cr}$.

N. Fk. Provo $R$

Humboldt $R$

Martin $\mathrm{Cr}$.

Trinity $\mathrm{R}$

$\begin{array}{llll}1596 & \text { I } & 1630 & 1911-30 \\ 101 & \text { II } & 94 & 1931- \\ 408 & \text { I } & 773 & 1911-65 \\ 128 & \text { I } & 316 & 1966- \\ 944 & \text { I } & 945 & 1916-30 \\ 459 & \text { I } & 434 & 1931- \\ 4927 & \text { I } & 5260 & 1911-16,1924-30 \\ 461 & \text { I } & 611 & 1931- \\ 464 & \text { I } & 380 & 1911-12,1915-21,1929 \\ 808 & \text { I } & 630 & 1922- \\ 2250 & \text { I } & 1080 & 1922- \\ 7580 & \text { II } & 301 & 1919-25,1929- \\ 2.0 & \text { I } & 0.83 & 1911-16 \\ 36.2 & \text { I } & 52.5 & 1917- \\ 1460 & \text { III } & 1258 & 1911- \\ 91 & \text { I } & 50.7 & 1915- \\ 53 & \text { I } & 12 & 1952- \\ 1450 & \text { II } & 352 & 1912- \\ 537 & \text { II } & 492 & 1911-30 \\ 67.9 & \text { I } & 141 & 1931- \\ & & & \end{array}$

\section{5}

117

65.3

559

337

208

496

6330

682

417

550

3271

3.01

6900

508

604

48.5

101

24.4

5010

172

726
II

I

I

I

I

I

I

I

II

II

I

I

II

I

I

I

II

III

II

III

II

III

II

II, III
1282 1919-

235 1911-18

117 1919-

796 1911-20

572 1921-

410 1911-15

$520 \quad 1916-$

$4820 \quad 1922-35,1940-$

$590 \quad 1924-29$

$351 \quad 1930-$

391 1911-12,1915-

$1500 \quad 1911-24$

1.35 1925-

1065 1912-15

179 1923-

$70.3 \quad 1931-$

430. 1911-

63.8 1911-68

$90.5 \quad 1914-$

35 1969-

359 1912-21

$31.9 \quad 1922-$

1729 1912- 
Table 1.--Gaging Station Records Used (continued)

\begin{tabular}{|c|c|c|c|c|c|c|}
\hline Cell & Number & River & $\begin{array}{l}\mathrm{Dr} \cdot \text { Area } \\
\left(\mathrm{mi}^{2}\right)\end{array}$ & Class & $\begin{array}{l}\text { Normal } \\
\left(f t^{3 / s}\right)\end{array}$ & $\begin{array}{l}\text { Record used } \\
\text { (water years) }\end{array}$ \\
\hline $10 \mathrm{~B}$ & 01076500 & Penngewasset $R$ & 622 & $I$ & 1350 & $1911-$ \\
\hline $10 \mathrm{C}$ & 01350000 & Schoharie Cr. & 236 & $\bar{I}$ & 462 & $1911-12$ \\
\hline $10 \mathrm{C}$ & 01321000 & Sacondaga $\mathrm{R}$ & 491 & I & 1080 & $1912-$ \\
\hline 10D & 04223000 & Genesee R & 981 & II & 1250 & $1911-$ \\
\hline $10 \mathrm{~F}$ & 04174500 & Huron R & 729 & I, II & 441 & $1911-$ \\
\hline $10 \mathrm{H}$ & 04073500 & Fox $\mathrm{R}$ & 1430 & II & 1031 & $1911-14$ \\
\hline $10 \mathrm{H}$ & 05436500 & Sugar $R$ & 523 & I & 314 & $1915-$ \\
\hline $10 \mathrm{I}$ & 05464500 & Cedar R & 6510 & I & 3021 & $1911-32$ \\
\hline $10 \mathrm{I}$ & 05459500 & Winnebago $\mathrm{Cr}$. & 526 & I & 199 & $1933-$ \\
\hline $10 \mathrm{~J}$ & 05480500 & Des Moines $R$ & 4190 & I & 1332 & $1914-27$ \\
\hline $10 \mathrm{~J}$ & 06600500 & Floyd R & 882 & I & 166 & $1936-$ \\
\hline $10 \mathrm{~K}$ & 06452000 & White $R$ & 10200 & II & 535 & 1929- \\
\hline $10 \mathrm{~L}$ & 06449500 & So. Fk. (Little) White R & 1020 & II & 104 & $1944-$ \\
\hline $10 \mathrm{M}$ & 06646500 & Deer $\mathrm{Cr}$ & 216 & III & 51.2 & 1916-60,(broken) \\
\hline $10 \mathrm{M}$ & 06649000 & $\mathrm{La}$ Prele $\mathrm{Cr}$ & 135 & III & 34 & $1920-71$ \\
\hline $10 \mathrm{M}$ & 06647500 & Box Elder Cr. & 63 & II & 35 & 1947-,(broken) \\
\hline 100 & 13011000 & Snake $\mathrm{R}$ & 824 & III & 1354 & $1911-18$ \\
\hline 100 & 06225000 & Bu11 Lake Cr. & 213 & I & 260 & $1919-37$ \\
\hline 100 & 09201000 & New Fork R & 552 & III & 371 & $1938-69$ \\
\hline $10 \mathrm{P}$ & 13083000 & Trapper $\mathrm{Cr}$ & 53.7 & II & 13.6 & $1912-16,1920-$ \\
\hline 10P & 13139000 & Big Wood (and Slough) & 640 & II & 426 & $1916-25$ \\
\hline $10 Q$ & 13185000 & Boise $R$ & 830 & I & 1158 & $1912-$ \\
\hline $10 R$ & 10393500 & Silvies $\mathrm{R}$ & 934 & I & 166 & $1911-,($ broken $)$ \\
\hline $10 \mathrm{~S}$ & 14321000 & Umpqua $\mathrm{R}$ & 3683 & II & 7500 & $1911-$ \\
\hline $10 \mathrm{~s}$ & 11483500 & Miller Cr. & 220 & III & 71 & $1926-50$ \\
\hline $10 \mathrm{~T}$ & 14325000 & So. Fk. Coquille $\mathrm{R}$ & 169 & II & 810 & $1917-26,1930-$ \\
\hline $11 \mathrm{~A}$ & 01031500 & Piscataquis $\mathrm{R}$ & 297 & I & 584 & $1911-$ \\
\hline $11 \mathrm{~B}$ & 04286500 & Dog $R$ & 52 & II & 80 & $1912-20,1929-34$ \\
\hline $11 \mathrm{~B}$ & 04287000 & Dog $R$ & 76.1 & II & 121 & $1935-$ \\
\hline $11 \mathrm{C}$ & 04269000 & St. Regis & 616 & $\mathrm{I}$ & 1004 & $1911-$ \\
\hline $11 \mathrm{E}$ & $02 \mathrm{FCO01}$ & Saugeen $R$ (Ontario) & 1570 & $\mathrm{I}$ & 1953 & $1915-$ \\
\hline $11 \mathrm{H}$ & 04077000 & Wolf $\mathrm{R}$ & 812 & II & 757 & $1911-13$ \\
\hline $11 \mathrm{H}$ & 0407.1000 & Oconto $\mathrm{R}$ & 678 & $\mathrm{I}$ & 515 & $1914-$ \\
\hline $11 \mathrm{I}$ & 05341500 & Apple $\mathrm{R}$ & 555 & II & 287 & $1911-70$ \\
\hline $11 \mathrm{I}$ & 05365500 & Chippewa R & 5600 & II & 4985 & $1971-$ \\
\hline $11 \mathrm{~J}$ & 05049000 & Mustinka $\mathrm{R}$ & 834 & II & 43.6 & $1916-24,1931-58$ \\
\hline $11 \mathrm{~J}$ & 05290000 & Little Mustinka $\mathrm{R}$ & 447 & I & 35.5 & $1959-$ \\
\hline $11 \mathrm{~K}$ & 06478500 & James $\mathrm{R}$ & 21550 & II & 335. & $1929-$ \\
\hline $11 \mathrm{~L}$ & 06441500 & Bad R & 3107 & $I$ & 162 & $1929-$ \\
\hline $11 \mathrm{M}$ & 06334000 & Little Missouri R & 904 & II & 60 & $1913-32,1936-69$ \\
\hline $11 \mathrm{~N}$ & 06275500 & Wood $\mathrm{R}$ & 218 & III & 132 & $1911-12,1915-16$ \\
\hline $11 \mathrm{~N}$ & 06207500 & Clarks Fork & 1340 & II & 897 & $1922-$ \\
\hline 110 & 06191500 & Yellowstone $\mathrm{R}$ & 2623 & II & 2865 & $1911-$ \\
\hline $11 Q$ & 13337000 & Lochsa $\mathrm{R}$ & 1180 & $\mathrm{I}$ & 2760 & $1911-12,1930-$ \\
\hline $11 \mathrm{R}$ & 13319000 & Grande Ronde R & 678 & II & 370 & $1911-,($ broken $)$ \\
\hline $11 \mathrm{~S}$ & 14159000 & McKenzie $\mathrm{R}$ & 348 & $\mathrm{I}, \mathrm{II}$ & 1650 & $1911-$ \\
\hline
\end{tabular}


able 1.--Gaging Station Records Used (continued)

\begin{tabular}{|c|c|c|c|c|c|}
\hline Number & River & $\begin{array}{l}\text { Dr. Area } \\
\left(\mathrm{mi}^{2}\right)\end{array}$ & Class & $\begin{array}{l}\text { Normal } \\
\left(\mathrm{ft}^{3} / \mathrm{s}\right)\end{array}$ & $\begin{array}{l}\text { Record used } \\
\text { (water years) }\end{array}$ \\
\hline 04024000 & St. Louis $\mathrm{R}$ & 3430 & III & 2200 & $1911-25$ \\
\hline 04014500 & Baptism R & 140 & I & 160 & $1928-$ \\
\hline 05054000 & Red R. of the $N$. & 6800 & III & 455 & $1911-$ \\
\hline 05082500 & Red R.(Intervening area) & 23300 & III & 1605 & $1911-$ \\
\hline 06345500 & Heart R & 1240 & I & 103 & $1911-22$ \\
\hline 06354000 & Cannonba11 R & 4100 & I & 233 & $1935-$ \\
\hline 06339500 & Knife R & 1230 & I & 94 & 1911-,(broken) \\
\hline 06177500 & Redwater $\mathrm{R}$ & 534 & II & 16.1 & $1932-71$, (broken) , 1975- \\
\hline 06061500 & Prick1y Pear Cr. & 175 & II & 45 & $1911-16$ \\
\hline 06110000 & Judith R & 331 & I & 45.3 & $1920-75$ \\
\hline 06109800 & So. Fk. Judith $\mathrm{R}$ & 58.7 & II & 16.0 & $1976-$ \\
\hline 06063000 & Tenmile $\mathrm{Cr}$. & 102 & II & 21 & $1911-14$ \\
\hline 06062500 & Tenmile $\mathrm{Cr}$. & 32.7 & III & 14.5 & $1915-$ \\
\hline 13339000 & Clearwater $\mathrm{R}$ & 4850 & I & 7930 & $1911-20$ \\
\hline 12413500 & Coeur $d^{\prime}$ Alene $R$ & 1220 & I & 2580 & $1912,1921-72$ \\
\hline 12413000 & Coeur $d^{\prime}$ Alene $R$ & 895 & I & 1950 & $1966-$ \\
\hline 12117500 & Cedar R & 125 & III & 680 & $1911-$ \\
\hline 12452500 & Chelan $R$ & 924 & III & 2081 & $1911-$ \\
\hline 14110000 & Klickitat $\mathrm{R}$ & 360 & II & 818 & $1911-71$ \\
\hline 12027500 & Chehalis $\mathrm{R}$ & 895 & II & 2850 & $1951-$ \\
\hline 04010500 & Pigeon $\mathrm{R}$ & 600 & I & 503 & $1924-$ \\
\hline 05129500 & Rainy $\mathrm{R}$ & 14900 & III & 9970 & $1911-28$ \\
\hline 05132000 & Big Fork & 1460 & I & 644 & $1929-$ \\
\hline 05MJ001 & Assiniboine $R$ & 59100 & II & 1577 & $1914-$ \\
\hline 05090000 & Park R & 695 & II & 48.5 & $1932-$ \\
\hline 05120500 & Wintering $\mathrm{R}$ & 675 & II & 11.9 & $1938-$ \\
\hline 06099500 & Marais $\mathrm{R}$ & 3242 & III & 920 & $1912-$ \\
\hline 06095000 & Birch $\mathrm{Cr}$. & 105 & III & 150 & $1911-22$ \\
\hline 12370000 & Swan $R$ & 671 & II & 1077 & $1923-$ \\
\hline 12303000 & Kootenau R & 10240 & II & 12100 & $1911-71$ \\
\hline 12304500 & Yaak R & 766 & II & 870 & $1972-$ \\
\hline $08 \mathrm{MHOO} 1$ & Chilliwack R (BC) & 474 & I & 2400 & $1972-$ \\
\hline 08MH016 & Chilliwack R & 127 & I & 680 & $1924-$ \\
\hline
\end{tabular}


miles. Table 1 shows the pertinent data for gaging stations used - drainage area, classification, normal flow, and period of record for which useful records were available and to which the indicated classification applies. Table 1 also shows the "cell" location based on the grid plan shown in figure 70. Each of the 165 cells has an area of about 23,000 square miles. The country (48 States) was divided into three regions as shown in figure 70, West, Center, and East, each region comprising about 55 cells.

The several stations were assigned to a cell depending on the latitude and longitude of the gage. Where, as was usually the case, records were not continuous over the whole 1911-79 period, different intervals may be represented by different stations. A cell may have more than one record for a given year and, in such cases, an average of the ratios to respective normal was calculated at each station.

The annual mean flows (by water years ) at the several gaging stations are each reported following past practice (Busby, 1963) in ratio to a reference normal, based in this report on the mean flow during the 1931-60 period. Since much work had already been done on the 1931-60 period, that reference period was retained for this work. It is important to use a single reference period throughout a given data set and study; however, none of the results would be changed by the adoption of any other period.

Comparison of the means for the period 1931-1960 with that for the period 1911-1979 for the 15 stations that are continuous for that 69-year period show the following consistent results:

$\begin{array}{lcc}\text { Region } & \begin{array}{r}\text { Number of } \\ \text { Stations }\end{array} & \begin{array}{r}\text { Ratio of means } \\ 1911-79 \text { to } 1931-60\end{array} \\ \text { West } & 6 & 1.06 \\ \text { Center } & 4 & 1.08 \\ \text { East } & 5 & 1.05\end{array}$

Figures 1-69 are computer plots of the annual ratios according to cell location. Annual ratios that are in the upper quartile of the ratios for each cell are noted by a superscript, and those that are in the lower quartile by a subscript. Unmarked ratios are deemed within the normal range; those marked by a superscript are termed "above normal" and those marked by a subscript are termed "below or subnormal." ( In earlier reports the terms "excessive" and "deficient" were used.) Table 2 lists, by years, the percentage of measured cells in each region, and for the U.S. ( 48 States) as a whole, that are subnormal as defined.

1/ The water year is the 12-month period beginning October 1 through September 30 of the succeeding calendar year.

2/ A 30-year normal follows international convention (WMO, 1956), and that for 1931-60 is nearly central within the period from 1911 to 1979 . 


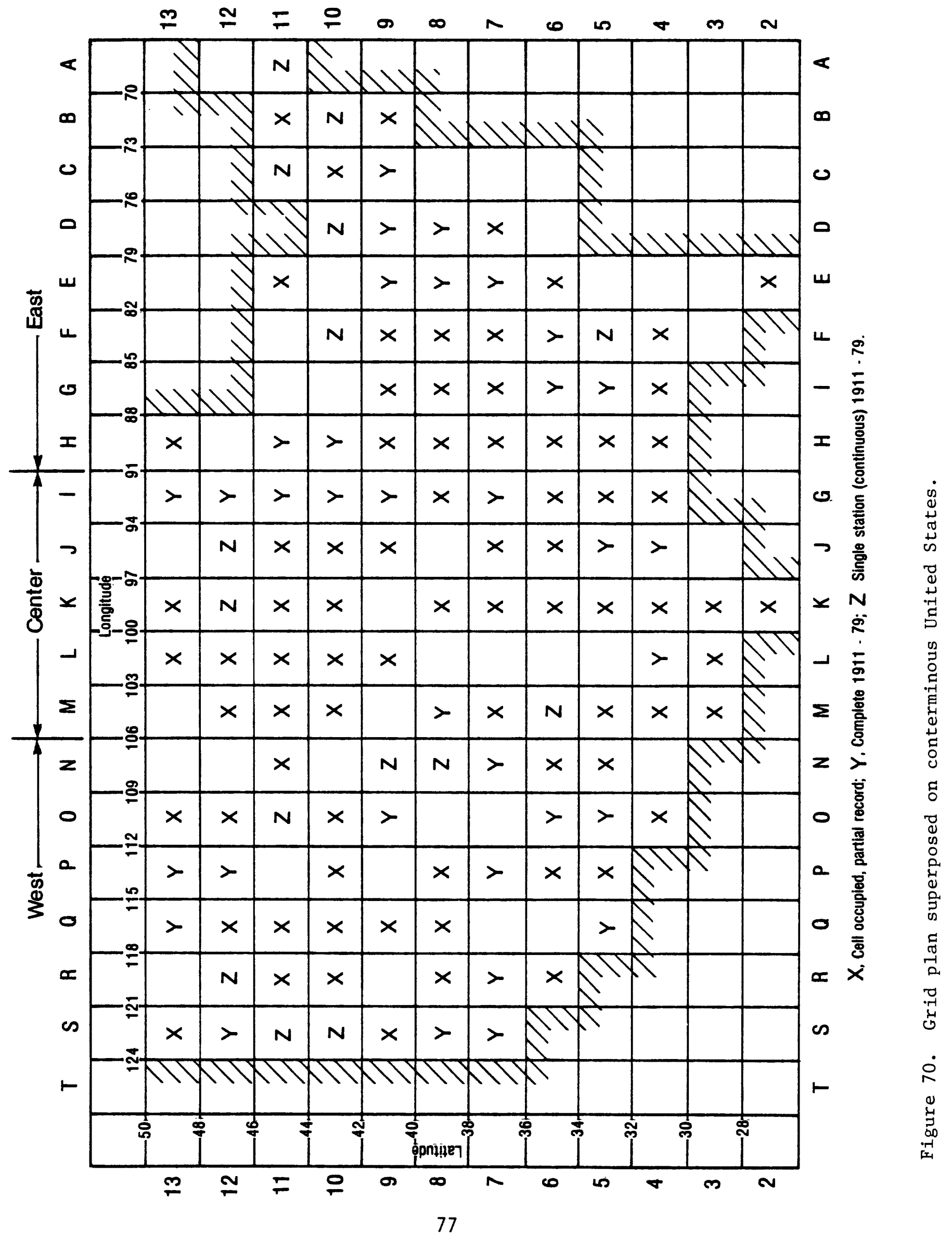


Table 3.--Number of dry years by decades and for each region and for the conterminous United States as a whole

$\begin{array}{lllll}\text { Decade } & \underline{W} & \underline{\mathrm{C}} & \underline{E} & \underline{\text { U.S. }} \\ 1911-20 & 1 & 3 & 3 & 3 \\ 1916-25 & 1 & 4 & 3 & 3 \\ 1921-30 & 4 & 2 & 4 & 4 \\ 1926-35 & 7 & 3 & 5 & 4 \\ 1931-40 & 7 & 6 & 5 & 5 \\ 1936-45 & 5 & 3 & 4 & 4 \\ 1941-50 & 3 & 0 & 2 & 1 \\ 1946-55 & 3 & 4 & 2 & 3 \\ 1951-60 & 5 & 6 & 5 & 5 \\ 1956-65 & 6 & 5 & 7 & 5 \\ 1961-70 & 5 & 4 & 8 & 5 \\ 1966-75 & 3 & 1 & 4 & 2 \\ 1971-79 & 3 & 2 & 0 & 2\end{array}$

large areal coverage of subnormal flows than are indicated on an assumption of independence. The reason for this difference seems to rest upon the coherence among ratios at the several cells within the regions, particularly in dry years. For example, 87 percent of cells in a region had subnormal runoff at least once in the 69-year period, whereas, if the distribution were random, only 42 percent of the cells would be subnormal, as a maximum, in the 69-year period.

Regional totals such as reported in table 3 can mask large local variations. For example, dividing the West region into north and south sections (divided at $42^{\circ} \mathrm{N}$. latitude) shows that each displays different variations. (See fig. 73.) The Northwest shows a peak in the number of dry years centering about the 1930's, whereas the Southwest shows its peak during the 1950's. Taken together, the West as a whole shows the two peaks common to the other regions. (See fig. 71.) However, the North-South contrast is more muted in the Center and East regions. The rough "cycle" suggested by the bar graphs in fig. 71 has of ten been noted in the precipitation in the United States, especially in the Great Plains, with 
Even casual inspection of the data in table 2 will suggest that there is a similar pattern of variation among the years in each of the regions. There are 26 years in which the areal percentages are on the same side of the U.S. median (20 percent) in each of the three regions, as against an expected frequency of about $17(=69 / 4)$. There is less than one chance in a hundred that this conformance among the regions would occur in random trials.

The data compiled by Beall (1978) for the upper Ohio River basin provide a test of the similarity in regimen among the annual flows in a cell. The upper Ohio River basin examined by Beall has an area of 27,300 square miles, about the same order of magnitude as a $2^{\circ}$ by $3^{\circ}$ cell used in this study and contains 98 long-term gaging stations. About 85 percent of the standard deviation of the individual records is accounted for by the annual basin means. The intra-class correlation coefficient (Fisher 1936, p. 216) is 0.72. Although as Beall noted ( $p .26$ ) there is a remarkable consistency in the annual ratios over a considerable part of that basin, particularly in the drier years, the cell is not monolithic in its behavior.

Figure 71 shows, by regions, bar graphs of the frequency of "dry" years by decades - a "dry" year being defined as one in which the extent of subnormal runoff exceeded the average. By definition, the average areal extent of subnormal runoff equals 25 percent, which is confirmed by averaging the data in the columns of table 2. To illustrate, the 1911-20 decade had one dry year in the West, 3 in the Center, and 3 in the East region. Table 3 lists the counts by decades and regions. In each region dry years as shown in figure 71 seem to be concentrated in two epochs at about a 25-year separation. The frequency of dry years during these peak periods is about 2 to 3 times that during intervening years. With reference to the data in table 3 , the variance among the decades (within the columns) is several times that among the regions (within the rows).

Busby (1963, table 5) shows data similar to that in table 2 for the conterminous U.S. as a whole. His figures for subnormal (= deficient) runoff are consistently less than those shown in table 2 of this report. His average of 0.18 (the same as obtained by Harbeck and Langbein, 1949, for the period 1921-45) seems significantly less than the 0.25 required by the definition of subnormal runoff. The difference may be due to the methods. In this report, the percent of subnormal runoff is the ratio of cells in the subnormal range for a given year to the number of cells for which data are available for that year. This procedure assumes that subnormal runoff among the ungaged cells occurs in the same ratio. In the earlier reports the subnormal area was defined from the areas shaded to designate the estimated areal extent, and as these areas were drawn more tightly about the known data, the percentages are lower. In the senior author's experience, the tendency was to view the ungaged area as being in the normal range.

The statistical distribution of the areal percentages for the regions given in table 2 is shown in figure 72 together with the theoretical ("random") distribution on the basis that subnormal flows occur 25 percent of the years at any cell by definition, and the assumption that the occurrence or nonoccurrence of subnormal flows in any cell is independent of the experience at any other. The actual distributions show a far greater number of large 
Table 2.--Percent of cells in each region with subnormal runoff by water years

Water

Year West Center East U.S.

$1911 \quad$ 8. 63. 65. 43.

1912 9. $\quad 6.00 .5$.

$1913 \quad 12 . \quad 22 . \quad 8 . \quad 13$.

$1914 \quad 3 . \quad 10 . \quad 32 . \quad 14$.

1915 42. $\quad 11 . \quad 25 . \quad 29$.

$1916 \quad 0 . \quad 5 . \quad 0 . \quad 1$.

$1917 \quad 3 . \quad 27 . \quad 18 . \quad 14$.

$1918 \quad 11 . \quad 43 . \quad 29.26$.

$1919 \quad 22 . \quad 13 . \quad 14 . \quad 17$.

$1920 \quad 17 . \quad 4 . \quad 14 . \quad 13$.

1921 6. 26. $15 . \quad 14$.

$1922 \quad 17 . \quad 8 . \quad 0.9$.

$1923 \quad 5 . \quad 21 . \quad 32 . \quad 18$.

$1924 \quad 53 . \quad 20 . \quad 10.29$.

$1925 \quad 3 . \quad 68 . \quad 68 . \quad 43$.

$1926 \quad 43 . \quad 23 . \quad 32.34$.

$1927 \quad 0.00 .82$.

$1928 \quad 8 . \quad 11.0 .6$.

1929 42. 0.6 6. 17.

1930 48. 22. 26. 33.

1931 80. $49 . \quad 82 . \quad 71$.

$1932 \quad 10.22 .40 .24$.

1933 32. 31. 5. 22.

1934 62. 78. 80.73.

193526.22.

$1936 \quad 17 . \quad 45 . \quad 33.31$.

$1937 \quad 45.20 .00 .22$.

$1938 \quad 2 . \quad 16.25$.

$1939 \quad 52 . \quad 36 . \quad 10.34$.

$1940 \quad 48 . \quad 70 . \quad 50.56$.

$194138 . \quad 18.40 .44$.

$1942 \quad 17 . \quad 2 . \quad 43.20$.

$1943 \quad 0 . \quad 16 . \quad 15 . \quad 10$.

$1944 \quad 48.4$ 4. 23. 24.

194514.11 .15$.
Water

Year West Center East U.S.

$1946 \quad 26 . \quad 20 . \quad 5 . \quad 17$.

1947 22. 4. 20. 15.

$1948 \quad 17 . \quad 18 . \quad 15 . \quad 17$.

1949 12. 7.20 .13$.

$1950 \quad 21 . \quad 9.20 . \quad 17$.

195124.29 .24$.

$1952 \quad 2 . \quad 20 . \quad 8 . \quad 10$.

$1953 \quad 23 . \quad 36.23 .27$.

1954 28. 56. 55. 46.

$1955 \quad 51 . \quad 29 . \quad 43.41$.

$1956 \quad 26 . \quad 59.40 .42$.

$1957 \quad 19 . \quad 18 . \quad 38.25$.

$1958 \quad 12 . \quad 23 . \quad 18 . \quad 17$.

$1959 \quad 33.43 .43 .40$.

$1960 \quad 38 . \quad 7 . \quad 5 . \quad 17$.

$1961 \quad 62 . \quad 30 . \quad 23 . \quad 38$.

$1962 \quad 14 . \quad 11.33 .19$.

1963 28. 52. 60.46.

$1964 \quad 37.41 .48 .42$.

$1965 \quad 5 . \quad 5 . \quad 40 . \quad 16$.

1966 33. 18. 50. 33.

$1967 \quad 12 . \quad 31 . \quad 38.27$.

196828.24 .23$.

$1969 \quad$ 5. $\quad 9 . \quad 28 . \quad 13$.

$1970 \quad 10 . \quad 18 . \quad 30.19$.

1971 13. 20. 10. 15.

1972 25. 21. 10.19.

$1973 \quad 49 . \quad 14 . \quad 0.21$.

$1974 \quad 18 . \quad 21.0 .0$.

$1975 \quad 10 . \quad 14 . \quad 3.9$.

$1976 \quad 35 . \quad 44 . \quad 10.30$.

1977 87. 51. 25. 54.

$1978 \quad 3 . \quad 15 . \quad 0.6$.

$1979 \quad 16.9$. 9.

West Center East U.S.

$\begin{array}{llll}\text { Median } & 19 . & 20 . & 20 .\end{array}$

Ave. 24. 24. 25.24.

Std. dev. 19. 18.21. 

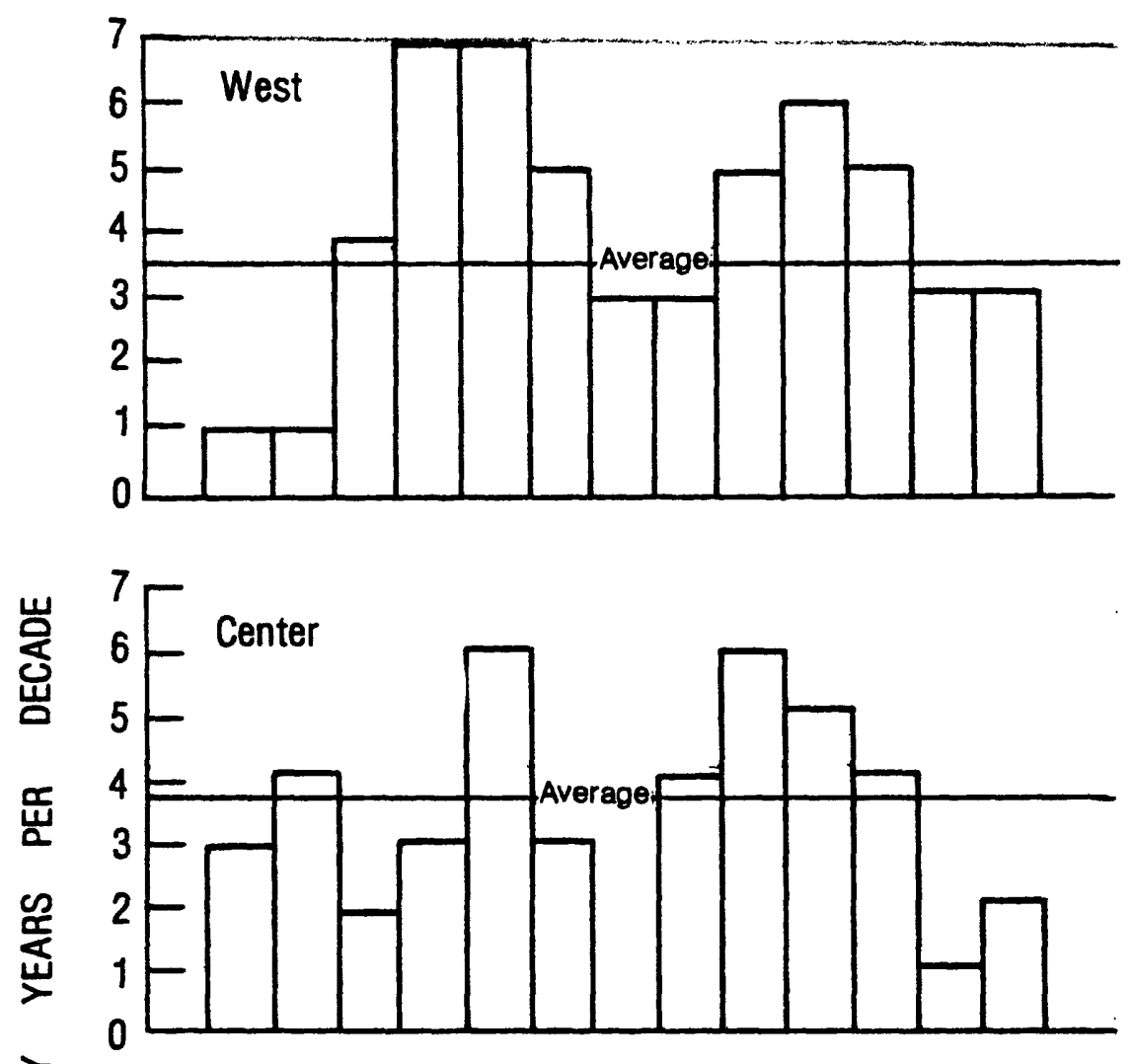

咅
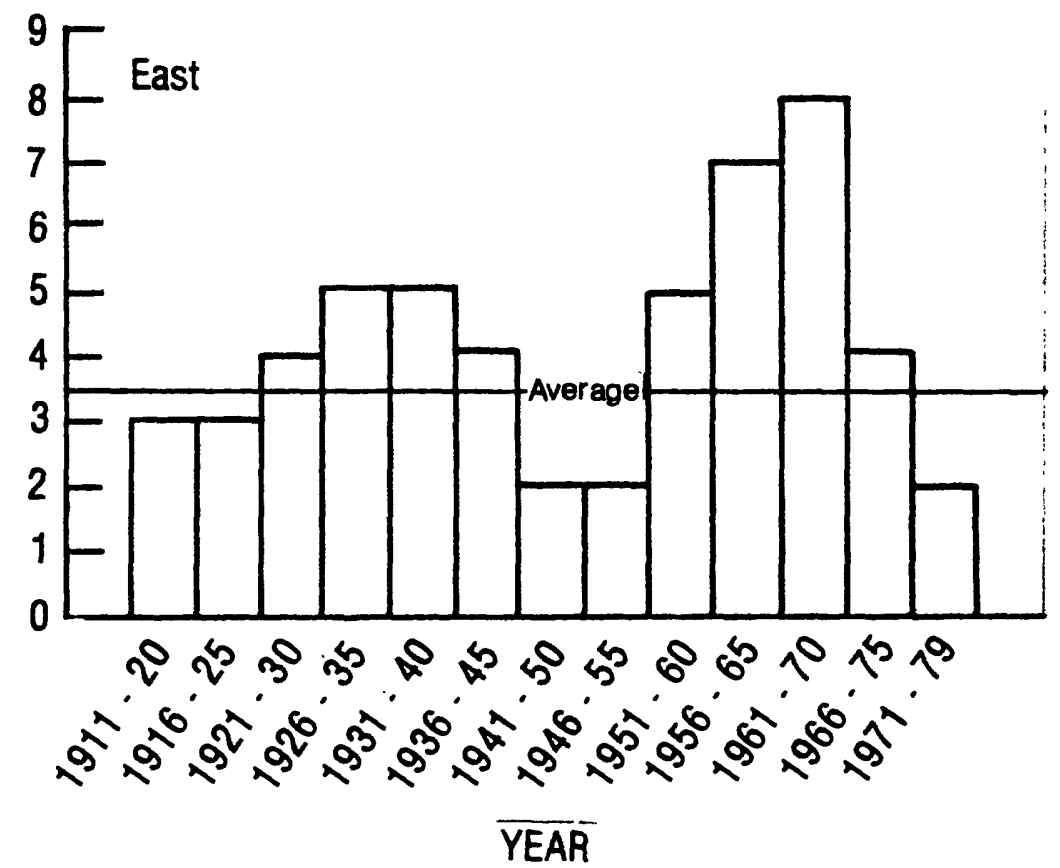

Figure 71. Frequency of dry years per decade, West, Center, and East regions. 


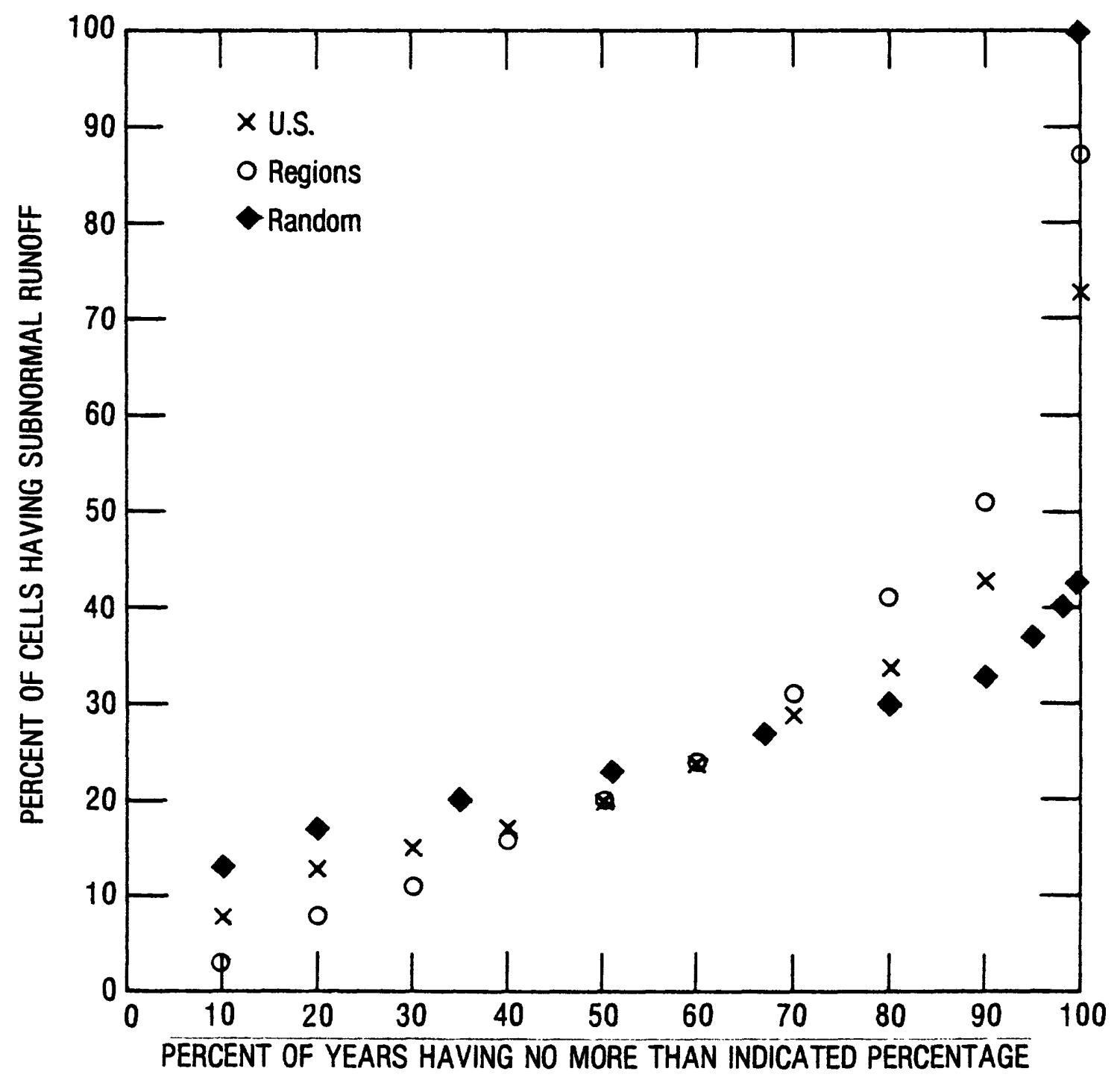

Figure 72. Distribution of years having indicated areal extent of subnormal runoff. 
"dry" periods occurring in 1895, 1915, 1935, and 1955. Borchert (1971) viewed this sequence to constitute sufficient grounds to warn that possible drought might follow that of the 1950's in the 1970's, and citing Thomas (1963, p. A40), who had observed that, "Nevertheless some climatic fluctuations appear to have recurrence intervals sufficiently regular, and amplitudes sufficiently great, that they may be important considerations in long-range planning for waterresources development and utilization." This study shows that similar fluctuations are present in the runoff throughout the country when aggregated regionally. The probability of dry years is not uniformly distributed through time. 


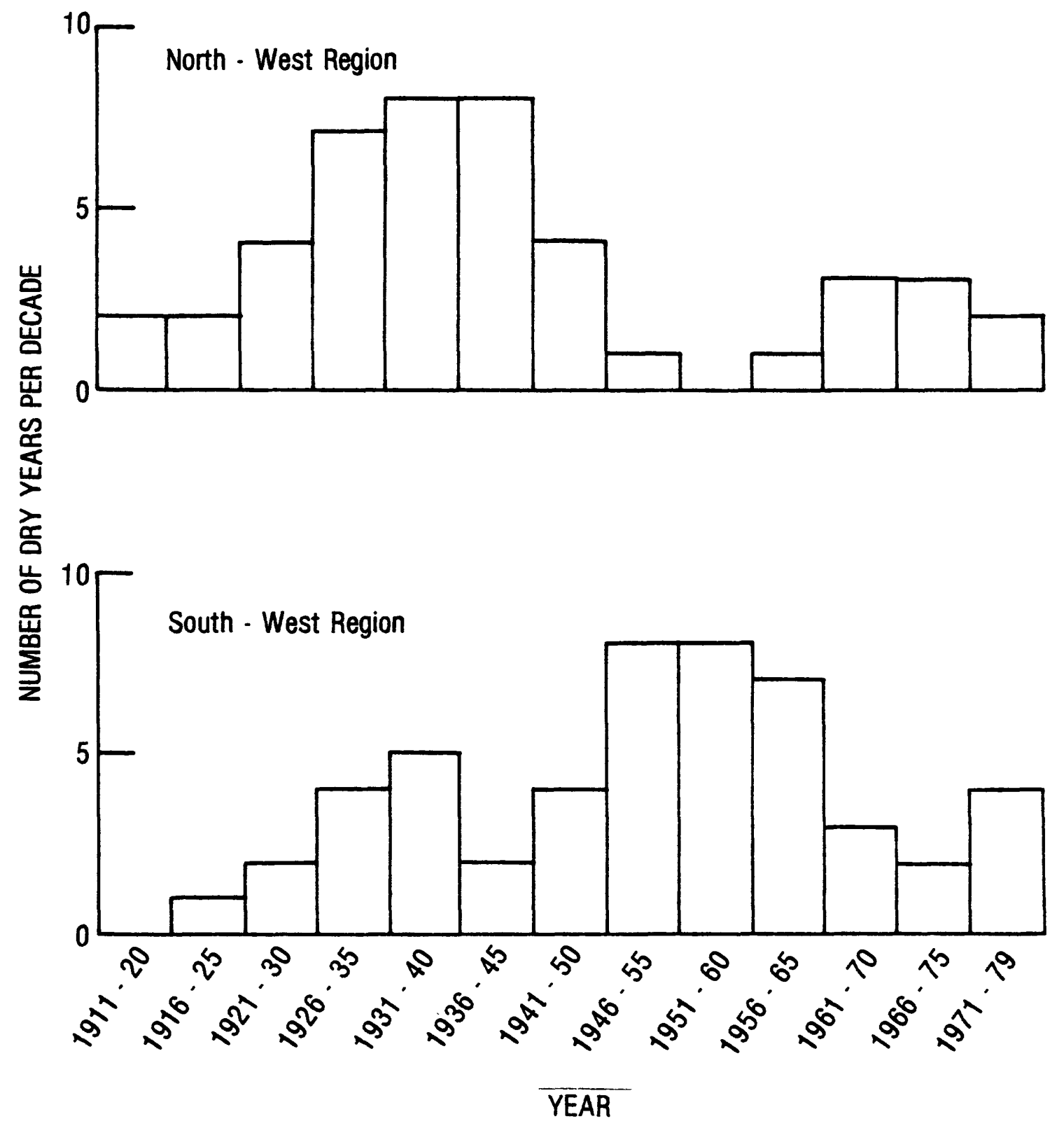

Figure 73. Frequency of dry years per decade in North and South parts of West region. 


\section{References}

Beal1, R. M., 1978, Mean annual runoff on the upper Ohio River basin, 1941-70, U.S. Geological Survey 2042, 32 p.

Borchert, J. R., 1971, The dust bow1 in the 1970's: Association of American Geographers, Annual, v. 61, p. 1-22.

Busby, M. W., 1963, Yearly variations in runoff for the conterminous United States, 1931-60: U.S. Geological Survey Water-Supply Paper 16695, 49 p.

Fisher, R. A., 1936, Statistical methods for research workers: 6th ed., O1iver \& Boyd, Edinburgh, Longon, 339 p.

Harbeck, G. E., and Langbein, W. B., 1949, Normals and variations in runoff 1921-1945: U.S. Geological Survey Water Resources Review, Supplement N.2, $35 \mathrm{p}$.

Langbein, W. B., and Slack, J. R., 1980, Variations in the national water supply 1979 update, Water Resources Review, January 1980, p. 18.

Thomas, H. E., and others, 1962, Drought in the southwest, 1942-56: U.S. Geological Survey Prof. Paper 372 (chapters $A$ to $\mathrm{H}$ issued and paged separately).

World Meterorological Organization, 1956, Technical regulations, Gen., lst ed., Geneva, Switzerland, Secretariat, World Meterological Orginization, v. 1, WMO-49. 